\title{
NAVIER-STOKES EQUATIONS ON THIN 3D DOMAINS. I: GLOBAL ATTRACTORS AND GLOBAL REGULARITY OF SOLUTIONS
}

\author{
GENEVIËVE RAUGEL AND GEORGE R. SELL
}

\section{CONTENTS}

1. Introduction

2. Notation: Statement of theorems

3. $H^{1}$-Regularity: Theorem 1

4. $H^{2}$-Regularity: Theorems 2,3

5. Reduced 3-dimensional theory: Theorem 6

6. Properties of attractors: Theorems 4, 5

7. Remarks on other boundary conditions: Theorem 7

8. Appendix: Proofs of auxiliary estimates

\section{INTRODUCTION}

The modern mathematical theory of fluid dynamics began over 50 years ago when Leray $(1933,1934 a, 1934 b)$ published his pioneering works on the NavierStokes equations. These equations describe the time evolution of solutions of mathematical models of viscid incompressible fluid flows. Because of this basic role in the modelling of fluid flows, there is considerable interest in developing a good mathematical theory of the behavior of the solutions of the Navier-Stokes equations. Since the solutions of these equations depend on both space and time, one is especially interested in the phenomenon of the time evolution of the spatial variations of the solutions. This phenomenon, which is described with more precision later, is referred to as the regularity of solutions, and it is the primary focus of the theory we present in this paper.

Received by the editors October 26, 1990.

1991 Mathematics Subject Classification. Primary 34C35, 35Q30, 76D05; Secondary 35K55, 58B99.

Key words and phrases. Attractor, global attractor, global regularity, Navier-Stokes equations, three-dimensional space.

This research was supported in part by grants from the Centre National de la Recherche Scientifique, the National Science Foundation, the Applied and Computational Mathematics Program/DARPA, and the Army Research Office. 
The Navier-Stokes equations on a bounded region $\Omega \subset \mathbb{R}^{n}, n=2,3$, are given by

$$
\begin{gathered}
U_{t}-\nu \Delta U+(U \cdot \nabla) U+\nabla P=F, \\
\nabla \cdot U=0,
\end{gathered}
$$

where $\nabla$ is the gradient operator and $\Delta$ is the Laplacian. In this paper we treat the case where $\Omega=\Omega_{\varepsilon}$ is a thin 3-dimensional domain, i.e., $\Omega_{\varepsilon}=Q_{2} \times$ $(0, \varepsilon)$, where $Q_{2}$ is a suitable bounded region in $R^{2}$ and $\varepsilon$ is a small positive parameter. In particular we will study $(1.1)$ with periodic boundary conditions where $Q_{2}=\left(0, l_{1}\right) \times\left(0, l_{2}\right)$, and $l_{1}$ and $l_{2}$ are positive.

Recall that the Navier-Stokes equations (1.1) on $\Omega$ can be written in the abstract form

$$
U^{\prime}+\nu A U+B(U, U)=\mathbb{P}_{n} F,
$$

where $\mathbb{P}_{n}$ is the orthogonal projection of $L^{2}\left(\Omega, \mathbb{R}^{n}\right)$ onto the space of divergence-free vector fields, $A U=-\mathbb{P}_{n} \Delta U$, and $B(U, V)=\mathbb{P}_{n}(U \cdot \nabla) V$. We will be interested in solutions of (1.2) under the assumption that the initial data $U_{0}$ satisfy

$$
U_{0} \in D\left(A^{1 / 2}\right),
$$

where $D\left(A^{1 / 2}\right)$ is the domain of $A^{1 / 2}$; see Temam $(1977,1983)$ and Constantin and Foias (1988). We also assume that the forcing function $F=F(t)$ satisfies

$$
F(\cdot) \in W^{1, \infty}\left([0, \infty), L^{2}(\Omega)\right) .
$$

In the case of periodic boundary conditions one has $D\left(A^{1 / 2}\right) \subset H_{\mathrm{per}}^{1}(\Omega)$. We will also assume, in this case, that

$$
\int_{\Omega} U_{0} d y=\int_{\Omega} F d y=0
$$

The phrase global regularity of solutions, or existence of strong solutions, refers to the property that when $U_{0}$ and $F$ satisfy (1.3) and (1.4), then (1.2) has a solution $U(t)$ that satisfies $U(0)=U_{0}$ and $U \in C^{0}\left([0, \infty), H^{1}(\Omega)\right)$. The principal outstanding problem for the 3-dimensional Navier-Stokes equations (3DNS) is to determine whether or not (1.2) has a global regular solution for every $U_{0}$ and $F$ satisfying (1.3) and (1.4).

The study of the regularity of solutions, both in 2-dimensions and 3-dimensions, has attracted widespread interest beginning with Leray $(1933,1934 \mathrm{a}$, 1934b). We are unable to give a complete history of this study here, but special mention should be made of the important contributions of Hopf (1951), Kiselev and Ladyzhenskaya (1957), Serrin (1962), Fujita and Kato (1964), Masuda (1967), Komatsu (1980), and Caffarelli, Kohn, and Nirenberg (1982). Additional references can be found in Giga (1988). Before describing our results on the global regularity of solutions of the 3DNS, let us review some aspects of the classical theory of regularity of these solutions.

For the 3DNS it is known that for every $U_{0}$ and $F$ satisfying (1.3) and (1.4) there is a $T$, which depends on $U_{0}$ and $F, 0<T \leq \infty$, such that (1.2) has a 
unique strong, or regular, solution $U(t)$ that satisfies $U \in C^{0}\left([0, T), H^{1}(\Omega)\right)$ $\cap L_{\text {loc }}^{2}((0, T), D(A))$ and $U^{\prime} \in L_{\text {loc }}^{2}\left((0, T), L^{2}(\Omega)\right)$. Furthermore, if the data $U_{0}$ and $F$ are small, then (as is known and as we show in $\S 2.11$ ) one has $T=\infty$, i.e., (1.2) has a globally regular solution for small data. Other than several theorems which establish the global regularity of solutions for small data, it is essentially unknown whether there are any other initial conditions $U_{0}$ and $F$ for which (1.2) has a globally regular solution, see Constantin and Foiaş (1988), Ladyzhenskaya (1969), Lions (1969), Temam (1977, 1983, 1988), and von Wahl (1985).

The theory of global regularity of solutions of the 2-dimensional NavierStokes equations (2DNS) is quite different. In this case there exists a globally regular solution of (1.2) for all $U_{0}$ and $F$ satisfying (1.3) and (1.4). Furthermore, one has $U(t) \in H^{2}(\Omega)$ for all $t>0$, and there exist positive constants $K$ and $L_{1}, L_{2}$ where $L_{1}$ and $L_{2}$ do not depend on $U_{0}$, such that

$$
\|U(t)\|_{H^{1}(\Omega)} \leq K, \quad 0 \leq t<\infty,
$$

and

$$
\limsup _{t \rightarrow \infty}\|U(t)\|_{H^{i}(\Omega)} \leq L_{i}, \quad i=1,2 .
$$

These classical results can be found in Ladyzhenskaya (1969), as well as in Constantin and Foias (1988) and Temam (1977, 1983, 1988). Because of the relevance of (1.5) and (1.6) for the 3-dimensional theory presented here, proofs of these relations are included in $\S 5$.

As a result of (1.5) and (1.6), it follows that in 2-dimensions, when $F$ is time independent, (1.2) has a global attractor $\mathfrak{A}$, and $\mathfrak{A}$ is a compact set in $H^{1}(\Omega)$ and compact in $H^{2}(\Omega)$; see Ladyzhenskaya (1972), Hale (1988), and Temam (1988). This means that $\mathfrak{A}$ is a Lyapunov stable attracting invariant $\operatorname{set}^{1}$ in $H^{1}(\Omega)$. If $F$ is time-varying, but has some compactness property (e.g., $F(t)$ is Bohr almost periodic in $t$ ), then by using the theory of skew-product flows (see Sacker and Sell $(1977,1990)$ and $\S 2.11)$ one can show that (1.2) generates a global attractor in $H^{1}(\Omega) \times \mathscr{F}$, where $\mathscr{F}$ is a compact positively invariant subset of $W^{1, \infty}\left([0, \infty), L^{2}(\Omega)\right)$.

It is the existence of the global attractor for the 2DNS which is the raison d'être of our study of the 3DNS on thin domains. Because a thin 3-dimensional domain is somehow close to a 2-dimensional domain, it is natural to ask whether one can use the good properties of the 2DNS to study the global regularity of the 3DNS. As we shall see, the theory presented here gives an affirmative answer to this question.

The idea of exploiting the existence of a global attractor of an evolutionary equation on an $n$-dimensional domain to obtain better information for a corresponding equation on a thin $(n+1)$-dimensional domain has already been used in Hale and Raugel (1990, 1992a, 1992b). Also see Raugel (1989).

\footnotetext{
${ }^{1}$ It is also the case that the global attractor $\mathfrak{A}$ for the 2DNS has finite dimension; see MalletParet (1976). The dimensionality of $\mathfrak{A}$ has been widely studied; see Temam (1988) for references to this literature.
} 
The process of exploiting the fact that $\Omega_{\varepsilon}$ is close to $Q_{2}$, when $\varepsilon$ is small, is far from being trivial. The main reason for the complication is due to the fact that the 3DNS on $\Omega_{\varepsilon}$ is a singular perturbation of the 2DNS on $Q_{2}$. The regularization of this singular perturbation is done in two steps, and it follows the methods introduced in Hale and Raugel (1992a, 1992b) for reaction diffusion equations and damped wave equations on thin domains. First one maps $\Omega_{\varepsilon}$ onto $Q_{3}=Q_{2} \times(0,1)$ by means of dilation. The Navier-Stokes equations (1.1) on $\Omega_{\varepsilon}$ are then transformed to the dilated Navier-Stokes equations on $Q_{3}$; see (2.4). This dilation alone does not remove the singular perturbation because some of the differential operators in (2.4) contain coefficients with $\varepsilon^{-1}$, or $\varepsilon^{-2}$, and $\varepsilon$ is small. Nevertheless, since the domain is now fixed to be $Q_{3}$ for all $\varepsilon>0$, this opens up the possibility of using other techniques from the theory of partial differential equations to regularize the singular perturbation in (2.4). The second step is accomplished by introducing the orthogonal projection $v=M u$ where

$$
v\left(x_{1}, x_{2}\right)=\int_{0}^{1} u\left(x_{1}, x_{2}, s\right) d s .
$$

By applying $M$ and $(I-M)$ to the dilated Navier-Stokes evolutionary equation (2.5) one finds an equivalent system (2.23). What we effectively show in this paper is that the system (2.23) is a regular perturbation of the 2DNS when $\varepsilon$ is small.

In Raugel and Sell (1989), we described the above method and announced some of our existence results. Since that time, we noticed that a more accurate Sobolev inequality given in Hale and Raugel (1992a) (also see the appendix, §8) can be used to improve the existence theorem in $H^{1}\left(\Omega_{\varepsilon}\right)$ in a significant way.

The following theorem is the principal result in this paper:

Theorem A. Consider the 3DNS (1.1) on $\Omega_{\varepsilon}$ with periodic boundary conditions. There is an $\varepsilon_{0}=\varepsilon_{0}\left(\nu, \lambda_{1}\right)>0$ such that for every $\varepsilon, 0<\varepsilon \leq \varepsilon_{0}$, there are large sets $\mathscr{R}(\varepsilon)$ and $\mathscr{S}(\varepsilon)$ where

$$
\begin{aligned}
& \mathscr{R}(\varepsilon) \subset\left\{U \in H^{1}\left(\Omega_{\varepsilon}\right): \nabla \cdot U=0, \int_{\Omega_{\varepsilon}} U d y=0\right\}, \\
& \mathscr{S}(\varepsilon) \subset\left\{F \in W^{1, \infty}\left([0, \infty), L^{2}\left(\Omega_{\varepsilon}\right)\right): \int_{\Omega_{\varepsilon}} F d y=0\right\},
\end{aligned}
$$

such that if $U_{0} \in \mathscr{R}(\varepsilon)$ and $F \in \mathscr{S}(\varepsilon)$, then (1.2) has a strong solution $U(t)$ with $U(0)=U_{0}$, defined for all $t \geq 0$, and

$$
\|U(t)\|_{H^{1}\left(\Omega_{\varepsilon}\right)}^{2} \leq \widehat{K}_{1}<\infty,
$$

where $\widehat{K}_{1}$ depends on $U_{0}$ and $F$. Furthermore, there exist constants $\widehat{L}_{1}$ and $\widehat{L}_{2}$, which do not depend on $U_{0}$ and which satisfy

$$
\limsup _{t \rightarrow \infty}\|U(t)\|_{H^{1}\left(\Omega_{\varepsilon}\right)} \leq \widehat{L}_{1}, \quad \limsup _{t \rightarrow \infty}\|U(t)\|_{H^{2}\left(\Omega_{\varepsilon}\right)} \leq \widehat{L}_{2} .
$$

The proof of Theorem A, including a clarification of the significance of the assertion that $\mathscr{R}(\varepsilon)$ and $\mathscr{S}(\varepsilon)$ are large sets, will be incorporated into the $H^{1}$ 
and $H^{2}$-Regularity Theorems, which are discussed in the next three sections. It is a consequence of these Regularity Theorems that the set of strong solutions of the Navier-Stokes evolutionary equation has a local attractor $\mathfrak{A}_{\varepsilon}=\mathfrak{A}_{\varepsilon}(F)$ whenever $F$ satisfies some compactness property and $F \in \mathscr{S}(\varepsilon)$. The basin of attraction of $\mathfrak{A}_{\varepsilon}$ contains the set $\mathscr{R}(\varepsilon) \times H^{+}(F)$; see $\S 2.11$. Moreover, we show that for $F \in \mathscr{S}(\varepsilon)$ the set $\mathfrak{A}_{\varepsilon}$ is the global attractor for the Leray solutions of the 3DNS on $\Omega_{\varepsilon}$, i.e., those weak solutions that satisfy the energy inequality (3.35). Furthermore, when $F$ is time-independent, $\mathfrak{A}_{\varepsilon}$ is a compact set in $H^{2}\left(\Omega_{\varepsilon}\right)$. We also show that, under reasonable assumptions, $\mathfrak{A}_{\varepsilon}$ is upper semicontinuous at $\varepsilon=0$.

In the next section we introduce the notation used in this paper, and we state the main theorems proved herein. The proofs of the regularity theorems will be given in $\S 3$ and $\S 4$, and the theory of the reduced 3DNS is presented in $\S 5$. The reduced 3DNS describe solutions of the 3DNS which depend only on two spatial coordinates. In $\S 6$ we study the attractor $\mathfrak{A}_{\varepsilon}$ for the 3DNS and we show that, under reasonable hypotheses, $\mathfrak{A}_{\varepsilon}$ is close to the global attractor $\mathfrak{A}_{0}$ of the reduced 3DNS.

The theory of the 3DNS on thin domains, which we present in $\S \S 2-6$, will be formulated in the context of spatially periodic boundary conditions; however, the methods we use are valid in other settings. In $\S 7$ we will show how the theory presented here can be extended to cover the Navier-Stokes equations with other homogeneous boundary conditions. In a forthcoming paper we will present the theory of global regularity for the Navier-Stokes equations with inhomogeneous boundary conditions on thin 3-dimensional domains, and we will consider other types of thin domains.

The results described in this paper were presented at the Workshop on Dynamical Systems Approaches to Turbulence held at the IMA at the University of Minnesota in May 1990. Related contributions appear in Raugel and Sell $(1989,1992 \mathrm{a}, 1992 \mathrm{~b})$. It should be noted that by imposing various symmetry conditions on the solutions of the 3DNS, one can show the global regularity of these solutions; see Ladyzhenskaya (1970) and Mahalov, Titi, and Leibovich (1990).

\section{Notation: StATEMENT OF THEOREMS}

The Navier-Stokes equations on a bounded region $\Omega$ in $\mathbb{R}^{n}, n=2,3$, are given by

$$
\begin{gathered}
U_{t}-\nu \Delta U+(U \cdot \nabla) U+\nabla P=F, \\
\nabla \cdot U=0,
\end{gathered}
$$

where $\nabla$ is the gradient operator and $\Delta$ is the Laplacian. In this paper we will be especially interested in the case where $n=3$ and $\Omega$ is a thin domain of the form $\Omega_{\varepsilon}=Q_{2} \times(0, \varepsilon)$, where $Q_{2}$ is a suitable bounded domain in $\mathbb{R}^{2}$ and $\varepsilon$ is a small positive number. In particular, we will assume that $Q_{2}=$ $\left(0, l_{1}\right) \times\left(0, l_{2}\right)$, where $l_{1}$ and $l_{2}$ are positive. We will assume that $\varepsilon \leq l_{2} \leq l_{1}$ and $0<\varepsilon \leq 1$ and that the solutions $U$ of (2.1) satisfy the periodic boundary 
conditions:

$$
\left\{\begin{array}{l}
U\left(y+l_{i} e_{i}, t\right)=U(y, t), \quad i=1,2, \\
U\left(y+\varepsilon e_{3}, t\right)=U(y, t),
\end{array}\right.
$$

where $\left\{e_{1}, e_{2}, e_{3}\right\}$ is the natural basis in $\mathbb{R}^{3}$. In addition, we will require that $F$ and the initial data $U_{0}$ satisfy

$$
\int_{\Omega_{\varepsilon}} F d y=\int_{\Omega_{\varepsilon}} U_{0} d y=0 .
$$

It then follows that any solution $U$ of (2.1) with $U(0)=U_{0}$ will also satisfy $\int_{\Omega_{e}} U d y=0$ for $t>0$. Set $Q_{3}=Q_{2} \times(0,1)$, and define $a=\left(a_{1}, a_{2}, a_{3}\right)$, where $a_{i}=l_{i}^{-1}, i=1,2,3$, and $l_{3}=1$. The change of variables $\left(y_{1}, y_{2}, y_{3}\right)$ $\mapsto\left(x_{1}, x_{2}, x_{3}\right)$ where $x_{i}=y_{i}, i=1,2$, and $x_{3}=\varepsilon^{-1} y_{3}$ maps $\Omega_{\varepsilon}$ onto $Q_{3}$.

We will present some aspects of the theory of the Navier-Stokes equations in this section. For further information, consult Constantin and Foias (1988), Ladyzhenskaya (1969), Lions (1969), Temam (1977, 1983), or von Wahl (1985).

2.1. Dilated Navier-Stokes equations. The linear operator $J_{\varepsilon}$ given by $U=$ $J_{\varepsilon} u$, where

$$
U\left(y_{1}, y_{2}, y_{3}\right)=u\left(y_{1}, y_{2}, \varepsilon^{-1} y_{3}\right),
$$

sets up a one-to-one correspondence between measurable functions on $\Omega_{\varepsilon}$ and measurable functions on $Q_{3}$. Furthermore, one has $J_{\varepsilon}\left(W^{k, p}\left(Q_{3}\right)\right)=W^{k, p}\left(\Omega_{\varepsilon}\right)$ for any Sobolev space $W^{k, p}$. We will need the identity

$$
\|U\|_{L^{p}\left(\Omega_{\varepsilon}\right)}^{p}=\varepsilon\|u\|_{L^{p}\left(Q_{3}\right)}^{p}, \quad 1 \leq p<\infty
$$

where $U=J_{\varepsilon} u$. We shall use capital Roman letters to denote functions on $\Omega_{\varepsilon}$ and lower case Roman letters for functions on $Q_{3}$.

We want to let $\varepsilon$ vary in our study of the solutions of (2.1). Rather than studying a fuxed equation on a variable domain, it is more convenient to fix the domain and permit the equation to vary. Therefore, we shall follow the construction in Hale and Raugel (1992a). In particular, by using the operator $J_{\varepsilon}$, the Navier-Stokes equations (2.1) are transformed to the following system on $Q_{3}$ :

$$
\begin{gathered}
u_{t}-\nu \Delta_{\varepsilon} u+\left(u \cdot \nabla_{\varepsilon}\right) u+\nabla_{\varepsilon} p=f, \\
\nabla_{\varepsilon} \cdot u=0,
\end{gathered}
$$

where $\nabla_{\varepsilon}=\left(D_{1}, D_{2}, \varepsilon^{-1} D_{3}\right), \Delta_{\varepsilon}=D_{1}^{2}+D_{2}^{2}+\varepsilon^{-2} D_{3}^{2}, D_{i}=\partial / \partial x_{i}, i=1,2,3$, $u=J_{\varepsilon}^{-1} U, f=J_{\varepsilon}^{-1} F$, and $p=J_{\varepsilon}^{-1} P$. We will refer to (2.4) as the dilated Navier-Stokes equations on $Q_{3}$. Because of the terms $\varepsilon^{-1} D_{3}$ and $\varepsilon^{-2} D_{3}^{2}$ where $\varepsilon$ is small, the system (2.4) is a singular perturbation of the two-dimensional Navier-Stokes equations.

2.2. Abstract formulation. The next step is to reformulate the initial value problem for (2.4) as an abstract nonlinear evolutionary equation on a suitable 
Hilbert space $H_{\varepsilon}$. The approach we use is an adaptation of the theory presented in Temam (1983).

Let $L^{2}\left(Q_{3}\right)=L^{2}\left(Q_{3}, \mathbb{R}^{3}\right)$ denote the collection of all functions $u: Q_{3} \rightarrow \mathbb{R}^{3}$ with the property that

$$
\int_{\Omega_{3}}|u|^{2} d x=\int_{Q_{3}} u \cdot u d x<\infty
$$

and let

$$
\|u\| \stackrel{\text { def }}{=}\|u\|_{L^{2}\left(Q_{3}\right)}=\left(\int_{Q_{3}}|u|^{2} d x\right)^{1 / 2}
$$

denote the usual norm. For $m=0,1,2, \ldots$ let the Sobolev spaces $H_{p}^{m}\left(Q_{3}\right)=$ $H_{p}^{m}\left(Q_{3}, \mathbb{R}^{3}\right)$ be the closure in $H^{m}\left(Q_{3}, \mathbb{R}^{3}\right)$ of those smooth functions that are periodic in space, i.e.,

$$
u\left(x+l_{i} e_{i}\right)=u(x), \quad i=1,2,3 .
$$

One then has $H_{p}^{0}\left(Q_{3}\right)=L^{2}\left(Q_{3}\right)$. Also the norm on $H_{p}^{m}\left(Q_{3}\right)$ is generated by the inner product

$$
(u, v)_{m}=\sum_{|\alpha| \leq m} \int_{Q_{3}} D^{\alpha} u \cdot D^{\alpha} v d x .
$$

Let $H_{\varepsilon}=H_{\varepsilon}\left(Q_{3}\right)$ denote the closure in $L^{2}\left(Q_{3}\right)$ of those smooth functions $u$ that are periodic on $Q_{3}$ and satisfy

$$
\int_{Q_{3}} u d x=0 \text { and } \nabla_{\varepsilon} \cdot u \stackrel{\text { def }}{=} D_{1} u_{1}+D_{2} u_{2}+\varepsilon^{-1} D_{3} u_{3}=0
$$

Let $\mathbb{P}_{\varepsilon}$ denote the orthogonal projection of $L^{2}\left(Q_{3}\right)$ onto $H_{\varepsilon}$. By applying $\mathbb{P}_{\varepsilon}$ to $(2.4)$ and using the fact that $\mathbb{P}_{\varepsilon}\left(\nabla_{\varepsilon} p\right)=0$, we obtain the following abstract nonlinear evolutionary equation on $H_{\varepsilon}$ :

$$
u^{\prime}+\nu A_{\varepsilon} u+B_{\varepsilon}(u, u)=\mathbb{P}_{\varepsilon} f,
$$

where $\partial u / \partial t=u^{\prime}, u=\mathbb{P}_{\varepsilon} u \in H_{\varepsilon}, A_{\varepsilon} u=-\mathbb{P}_{\varepsilon} \Delta_{\varepsilon} u$ (with the periodic boundary conditions), and the bilinear form $B_{\varepsilon}$ satisfies $B_{\varepsilon}(u, v)=\mathbb{P}_{\varepsilon}\left(u \cdot \nabla_{\varepsilon}\right) v$. We shall refer to (2.5) as the dilated Navier-Stokes evolutionary equation. We define $V_{\varepsilon}^{m}$ for $m=0,1,2, \ldots$ by

$$
V_{\varepsilon}^{m}=H_{\varepsilon} \cap H_{p}^{m}\left(Q_{3}\right)
$$

Thus $V_{\varepsilon}^{0}=H_{\varepsilon}$. Also $A_{\varepsilon}$ is a selfadjoint operator with compact resolvent, and one has $D\left(A_{\varepsilon}\right)=V_{\varepsilon}^{2}$ and $D\left(A_{\varepsilon}^{1 / 2}\right)=V_{\varepsilon}^{1}$. Furthermore, $A_{\varepsilon}$ satisfies

$$
\lambda_{1}\|u\|^{2} \leq\left\|A_{\varepsilon}^{1 / 2} u\right\|^{2}, \quad u \in D\left(A_{\varepsilon}^{1 / 2}\right),
$$

where $\lambda_{1}>0$ is the smallest eigenvalue of $A_{\varepsilon}$. Since $0<\varepsilon \leq l_{2} \leq l_{1}$, one has $\lambda_{1}=4 \pi^{2} a_{1}^{2}=4 \pi^{2} l_{1}^{-2}$.

The evolutionary equation (2.5) does not contain the pressure term $\nabla_{\varepsilon} p$. In order to recover the pressure term we apply $\left(I-\mathbb{P}_{\varepsilon}\right)$ to $(2.4)$ to obtain

$$
\left(I-\mathbb{P}_{\varepsilon}\right)\left(u_{t}-\nu \Delta_{\varepsilon} u+\left(u \cdot \nabla_{\varepsilon}\right) u\right)+\nabla_{\varepsilon} p=\left(I-\mathbb{P}_{\varepsilon}\right) f,
$$


or in the case of periodic boundary conditions

$$
\left(I-\mathbb{P}_{\varepsilon}\right)\left(u \cdot \nabla_{\varepsilon}\right) u+\nabla_{\varepsilon} p=\left(I-\mathbb{P}_{\varepsilon}\right) f .
$$

If $u \in V_{\varepsilon}^{1}$ and $f \in L^{2}\left(Q_{3}\right)$ are known, one can solve (2.7) or (2.8) for $p$ by classical techniques; see Constantin and Foiaş (1988) and Temam (1977, 1983).

We will assume the forcing term $f$ in (2.4) to be a time-varying function in the space $L^{\infty}\left((0, \infty), L^{2}\left(Q_{3}\right)\right)$, and we define the norm $\|f\|_{\infty}$ by

$$
\|f\|_{\infty} \stackrel{\text { def }}{=} \underset{0<t<\infty}{\operatorname{ess} \sup }\|f(t)\|_{L^{2}\left(Q_{3}\right)} \text {. }
$$

For some applications, we will assume that $f \in W^{1, \infty}\left([0, \infty), L^{2}\left(Q_{3}\right)\right)$, in which case the function $f$ is absolutely continuous and the mapping $t \rightarrow f(t)$ is uniformly continuous.

We shall say that $u(t)$ is a strong solution of $(2.5)$ on an interval $[0, T)$, where $0<T \leq \infty$, if for every $\tau, 0<\tau<T$, one has

$$
u(\cdot) \in C^{0}\left([0, \tau], V_{\varepsilon}^{1}\right) \cap L^{2}\left((0, \tau), V_{\varepsilon}^{2}\right) .
$$

Recall that if $u(t)$ is a strong solution of $(2.5)$ on $[0, T)$, then it is uniquely determined; see Temam $(1977,1983)$ or Constantin and Foiaş (1988). Furthermore, if $u(t)$ is a solution of $(2.5)$ on an interval $[0, T)$, where $0<T \leq \infty$, and satisfies $u(\cdot) \in C^{0}\left([0, \tau], V_{\varepsilon}^{1}\right)$ for every $\tau, 0<\tau<T$, then $u(\cdot) \in$ $C^{0}\left((0, \tau], V_{\varepsilon}^{2}\right)$. (See $\S 4$. $)$

A strong solution $u(t)$ on an interval $[0, T)$ is said to be maximally defined if $u(t)$ does not have a proper extension to a strong solution of (2.5) on a larger interval. Recall that if $u(t)$ is a maximally defined strong solution of (2.5) on an interval $[0, T)$ and if $T<\infty$, then one has

$$
\left\|A_{\varepsilon}^{1 / 2} u(t)\right\| \rightarrow \infty \quad \text { as } t \rightarrow T^{-}
$$

see Temam $(1977,1983)$, or Constantin and Foiaş (1988).

2.3. Fourier series. The spaces $V_{\varepsilon}^{m}$ can also be described in terms of the Fourier series expansion for functions $u \in L^{2}\left(Q_{3}\right)$. For $k$ in the integer lattice $\mathbb{Z}^{3}$, we define

$$
k a \stackrel{\text { def }}{=}\left(k_{1} a_{1}, k_{2} a_{2}, k_{3} a_{3}\right) .
$$

Then the Fourier series expansion for $u \in L^{2}\left(Q_{3}\right)$ is given by

$$
u(x)=\sum_{k \in \mathbb{Z}^{3}} c^{k} e^{2 \pi i k a \cdot x}
$$

where $c^{k} \in \mathbb{C}^{3}, \overline{c^{k}}=c^{-k}$, and

$$
c^{k}=a_{1} a_{2} a_{3} \int_{Q_{3}} u(x) e^{-2 \pi i k a \cdot x} d x, \quad k \in \mathbb{Z}^{3} .
$$

Consequently, one has $u \in V_{\varepsilon}^{0}=H_{\varepsilon}$ if and only if $c^{0}=0$ and

$$
k_{1} a_{1} c_{1}^{k}+k_{2} a_{2} c_{2}^{k}+\varepsilon^{-1} k_{3} a_{3} c_{3}^{k}=0 \text { for all } k \in \mathbb{Z}^{3} \text {. }
$$


Similarly one has $u \in V_{\varepsilon}^{m}$ for $m \geq 0$ if and only if $c^{0}=0$, condition (2.12) holds, and

$$
\sum_{k \in \mathbb{Z}^{3}}|k|^{2 m}\left|c^{k}\right|^{2}<\infty
$$

where $|k|^{2}=k_{1}^{2}+k_{2}^{2}+k_{3}^{2}$. Furthermore, it follows from the Parseval equality that

$$
\|u\|_{H_{p}^{m}\left(Q_{3}\right)}^{2}=\left(a_{1} a_{2} a_{3}\right)^{-1} \sum_{|\alpha| \leq m} \sum_{k \in \mathbb{Z}^{3}}\left|(2 \pi)^{|\alpha|}(k a)^{\alpha} \cdot c^{k}\right|^{2}, \quad u \in H_{p}^{m}\left(Q_{3}\right),
$$

where $\alpha=\left(\alpha_{1}, \alpha_{2}, \alpha_{3}\right) \in \mathbb{N}^{3}, \mathbb{N}=\{0,1,2, \ldots\}$, and

$$
(k a)^{\alpha} \cdot c^{k}=\left(k_{1} a_{1}\right)^{\alpha_{1}} c_{1}^{k}+\left(k_{2} a_{2}\right)^{\alpha_{2}} c_{2}^{k}+\left(k_{3} a_{3}\right)^{\alpha_{3}} c_{3}^{k} \text {. }
$$

The eigenvalues of $A_{\varepsilon}$ are given by

$$
\lambda=4 \pi^{2}\left[\left(k_{1} a_{1}\right)^{2}+\left(k_{2} a_{2}\right)^{2}+\varepsilon^{-2}\left(k_{3} a_{3}\right)^{2}\right],
$$

where $k \in \mathbb{Z}^{3}-\{(0,0,0)\}$. If $u \in V_{\varepsilon}^{2}=D\left(A_{\varepsilon}\right)$, then one has

$$
A_{\varepsilon} u=4 \pi^{2} \sum_{k \in \mathbb{Z}^{3}}\left[\left(k_{1} a_{1}\right)^{2}+\left(k_{2} a_{2}\right)^{2}+\varepsilon^{-2}\left(k_{3} a_{3}\right)^{2}\right] c^{k} e^{2 \pi i k a \cdot x} .
$$

By using the Fourier series representation, it is easily verified that if $u \in V_{\varepsilon}^{2}$, then $\nabla_{\varepsilon} \cdot \Delta_{\varepsilon} u=0$. This implies that $A_{\varepsilon} u=-\Delta_{\varepsilon} u$ for all $u \in D\left(A_{\varepsilon}\right)$.

The Navier-Stokes equations $(2.1)$ on $\Omega_{\varepsilon}$ can be written in the abstract form

$$
U^{\prime}+\nu A U+B(U, U)=\mathbb{P}_{3} F,
$$

where $\mathbb{P}_{3}$ is the orthogonal projection of $L^{2}\left(\Omega_{\varepsilon}\right)$ onto the space of divergencefree vector fields, $A U=-\mathbb{P}_{3} \Delta U$, and $B(U, V)=\mathbb{P}_{3}(U \cdot \nabla) V$; cf. Temam (1983). One can use the operator $J_{\varepsilon}$ given by (2.2) to compare the solutions of (2.14) with those of (2.5). For example, if $U=J_{\varepsilon} u$, where $u$ is given by (2.11), then $U$ has the Fourier expansion

$$
U(y)=\sum_{k \in \mathbb{Z}^{3}} c^{k} e^{2 \pi i\left(k_{1} a_{1}, k_{2} a_{2}, \varepsilon^{-1} k_{3} a_{3}\right) \cdot\left(y_{1}, y_{2}, y_{3}\right)} .
$$

The following identities are easily verified:

$$
\frac{\partial}{\partial y_{i}} J_{\varepsilon} u=J_{\varepsilon} \varepsilon^{-\{i\}} \frac{\partial}{\partial x_{i}} u, \quad i=1,2,3, \text { for } u \in W^{1, p}\left(Q_{3}\right),
$$

where $\{1\}=\{2\}=0,\{3\}=1$. Also one has

$$
\Delta J_{\varepsilon} u=J_{\varepsilon} \Delta_{\varepsilon} u, \quad A J_{\varepsilon} u=J_{\varepsilon} A_{\varepsilon} u \quad \text { for } u \in D\left(A_{\varepsilon}\right) .
$$

As a consequence of (2.3) one then obtains

$$
\begin{cases}\left\|\partial U / \partial y_{i}\right\|_{L^{2}\left(\Omega_{\varepsilon}\right)}^{2}=\varepsilon\left\|\varepsilon^{-\{i\}} \partial u / \partial x_{i}\right\|_{L^{2}\left(Q_{3}\right)}^{2}, & u \in H^{1}\left(Q_{3}\right) \\ \left\|A^{r} U\right\|_{L^{2}\left(\Omega_{\varepsilon}\right)}^{2}=\varepsilon\left\|A_{\varepsilon}^{r} u\right\|_{L^{2}\left(Q_{3}\right)}^{2}, & u \in D\left(A_{\varepsilon}^{r}\right), \quad r \geq 0\end{cases}
$$


If $u$ is given by $(2.11)$ and belongs to $D\left(A_{\varepsilon}^{1 / 2}\right)$, we have

$$
\left\|A_{\varepsilon}^{1 / 2} u\right\|^{2}=\left(A_{\varepsilon} u, u\right)_{0}=4 \pi^{2}\left(a_{1} a_{2} a_{3}\right)^{-1} \sum_{k \in \mathbb{Z}^{3}}\left(k_{1}^{2} a_{1}^{2}+k_{2}^{2} a_{2}^{2}+\varepsilon^{-2} k_{3}^{2} a_{3}^{2}\right)\left|c^{k}\right|^{2} \text {. }
$$

Moreover, if $u$ belongs to $D\left(A_{\varepsilon}\right)$, one has

$$
\left\|A_{\varepsilon} u\right\|^{2}=16 \pi^{4}\left(a_{1} a_{2} a_{3}\right)^{-1} \sum_{k \in \mathbb{Z}^{3}}\left(k_{1}^{2} a_{1}^{2}+k_{2}^{2} a_{2}^{2}+\varepsilon^{-2} k_{3}^{2} a_{3}^{2}\right)^{2}\left|c^{k}\right|^{2} .
$$

From the Parseval equality (2.13), we conclude that there exist two positive constants $C_{6}$ and $C_{7}$, which are independent of $\varepsilon$, such that

$$
\begin{aligned}
C_{6}\left(\|u\|_{H^{1}\left(Q_{3}\right)}+\varepsilon^{-1}\left\|D_{3} u\right\|_{L^{2}\left(Q_{3}\right)}\right) & \leq\left\|A_{\varepsilon}^{1 / 2} u\right\|_{L^{2}\left(Q_{3}\right)} \\
& \leq C_{7}\left(\|u\|_{H^{1}\left(Q_{3}\right)}+\varepsilon^{-1}\left\|D_{3} u\right\|_{L^{2}\left(Q_{3}\right)}\right)
\end{aligned}
$$

and

$$
\begin{aligned}
& C_{6}\left(\|u\|_{H^{2}\left(Q_{3}\right)}+\varepsilon^{-1}\left\|D_{3} u\right\|_{H^{1}\left(Q_{3}\right)}+\varepsilon^{-2}\left\|D_{3}^{2} u\right\|_{L^{2}\left(Q_{3}\right)}\right) \\
& \quad \leq\left\|A_{\varepsilon} u\right\|_{L^{2}\left(Q_{3}\right)} \\
& \quad \leq C_{7}\left(\|u\|_{H^{2}\left(Q_{3}\right)}+\varepsilon^{-1}\left\|D_{3} u\right\|_{H^{1}\left(Q_{3}\right)}+\varepsilon^{-2}\left\|D_{3}^{2} u\right\|_{L^{2}\left(Q_{3}\right)}\right) .
\end{aligned}
$$

From (2.15) and (2.18), we deduce that $U=J_{\varepsilon} u$ satisfies

$$
C_{6}\|U\|_{H^{2}\left(\Omega_{\varepsilon}\right)} \leq\|A U\|_{L^{2}\left(\Omega_{\varepsilon}\right)} \leq 3 C_{7}\|U\|_{H^{2}\left(\Omega_{\varepsilon}\right)} .
$$

2.4. The projection $M$. For any $u \in L^{2}\left(Q_{3}\right)$ we define $v=M u$ by

$$
v\left(x_{1}, x_{2}\right)=\int_{0}^{1} u\left(x_{1}, x_{2}, s\right) d s
$$

and set $w=(I-M) u$. Since $w=(I-M) u$, one has $M w=0$, and $M$ is an orthogonal projection on $L^{2}\left(Q_{3}\right)$ which satisfies

$$
\begin{gathered}
M D_{i} u=D_{i} M u, \quad i=1,2, \text { for all } u \in W^{1,1}\left(Q_{3}\right), \\
M D_{3} u=D_{3} M u=0 \quad \text { for all } u \in H_{p}^{1}\left(Q_{3}\right),
\end{gathered}
$$

and, therefore,

$$
\nabla_{\varepsilon} \cdot M u=M \nabla_{\varepsilon} \cdot u \text { for all } u \in H_{p}^{1}\left(Q_{3}\right)
$$

as well as

$$
A_{\varepsilon} M u=M A_{\varepsilon} u \text { for all } u \in D\left(A_{\varepsilon}\right) \text {. }
$$

As a consequence of all these properties, we conclude that $M\left(V_{\varepsilon}^{m}\right) \subset V_{\varepsilon}^{m}$ and that $M$ is an orthogonal projection in $V_{\varepsilon}^{m}$ for all integers $m \geq 0$. In particular, we have

$$
\left\|A_{\varepsilon}^{r} u\right\|^{2}=\left\|A_{\varepsilon}^{r} v\right\|^{2}+\left\|A_{\varepsilon}^{r} w\right\|^{2}, \quad r=0, \frac{1}{2}, 1
$$


In terms of the Fourier series

$$
w(x)=\sum_{k \in \mathbb{Z}^{3}} c^{k} e^{2 \pi i k a \cdot x}
$$

one has $M w=0$ if and only if

$$
c^{\left(k_{1}, k_{2}, 0\right)}=0 \text { for all }\left(k_{1}, k_{2}\right) \in \mathbb{Z}^{2} .
$$

To put it another way, if $c^{k}$ is any nonzero Fourier coefficient for $w$, where $M w=0$, then $k=\left(k_{1}, k_{2}, k_{3}\right)$ satisfies $k_{3} \neq 0$. Consequently, one has the Poincaré inequality

$$
\|w\|^{2} \leq C_{5}^{2} \varepsilon^{2}\left\|A_{\varepsilon}^{1 / 2} w\right\|^{2}, \quad w \in V_{\varepsilon}^{1}, M w=0
$$

where $C_{5}$ does not depend on $\varepsilon$. Indeed, from the Parseval equality (2.13) with $m=0$ and from (2.16) one obtains

$$
\begin{aligned}
\left\|A_{\varepsilon}^{1 / 2} w\right\|_{L^{2}\left(Q_{3}\right)}^{2} & =4 \pi^{2}\left(a_{1} a_{2} a_{3}\right)^{-1} \sum_{k \in \mathbb{Z}^{3}}\left(k_{1}^{2} a_{1}^{2}+k_{2}^{2} a_{2}^{2}+\varepsilon^{-2} k_{3}^{2} a_{3}^{2}\right)\left|c^{k}\right|^{2} \\
& =4 \pi^{2}\left(a_{1} a_{2} a_{3}\right)^{-1} \sum_{k_{3} \neq 0}\left(k_{1}^{2} a_{1}^{2}+k_{2}^{2} a_{2}^{2}+\varepsilon^{-2} k_{3}^{2} a_{3}^{2}\right)\left|c^{k}\right|^{2} \\
& \geq 4 \pi^{2} \varepsilon^{-2}\left(a_{1} a_{2} a_{3}\right)^{-1} \sum_{k \in \mathbb{Z}^{3}}\left|c^{k}\right|^{2}=4 \pi^{2} \varepsilon^{-2}\|w\|_{L^{2}\left(Q_{3}\right)}^{2}
\end{aligned}
$$

which completes the proof of (2.22). As we shall see, (2.22) plays a critical role in the theory presented here.

2.5. The $v$ and $w$ equations. We now apply the projections $M$ and $(I-M)$ to the equation (2.5) where $v=M u$ and $w=(I-M) u$. Since one has $M B_{\varepsilon}(v, v)=B_{\varepsilon}(v, v)$, it follows from (2.20) that one obtains the system (2.23)

$$
\left\{\begin{array}{l}
v^{\prime}+\nu A_{\varepsilon} v+B_{\varepsilon}(v, v)=M \mathbb{P}_{\varepsilon} f-M\left(B_{\varepsilon}(v, w)+B_{\varepsilon}(w, v)+B_{\varepsilon}(w, w)\right), \\
w^{\prime}+\nu A_{\varepsilon} w=(I-M) \mathbb{P}_{\varepsilon} f-(I-M)\left(B_{\varepsilon}(v, w)+B_{\varepsilon}(w, v)+B_{\varepsilon}(w, w)\right) .
\end{array}\right.
$$

Since $v$ does not depend on $x_{3}$, one has $A_{\varepsilon} v=D_{1}^{2} v+D_{2}^{2} v$, i.e., $A_{\varepsilon} v$ is independent of $\varepsilon$. Similarly $B_{\varepsilon}(v, v)$ is independent of $\varepsilon$. The initial condition $u(0)=u_{0}=v_{0}+w_{0}$ also splits into a $v$ and $w$ component. We will be studying solutions $(v, w)=(v(t), w(t))$, where $v(0)=v_{0}=M u_{0}$ and $w(0)=w_{0}=(I-M) u_{0}$.

2.6. Reduced 3D Navier-Stokes evolutionary equation. The system (2.23) has an invariant set which occurs when

$$
(I-M) \mathbb{P}_{\varepsilon} f=0, \quad w_{0}=0,
$$

i.e., both the forcing term $\mathbb{P}_{\varepsilon} f$ and the initial condition $u_{0}$ depend only on $x_{1}$ and $x_{2}$. In this case $w(t) \equiv 0$ for all $t \geq 0$ and $\bar{v}=v(t)$ satisfies

$$
\bar{v}^{\prime}+\nu A_{\varepsilon} \bar{v}+B_{\varepsilon}(\bar{v}, \bar{v})=M \mathbb{P}_{\varepsilon} f
$$


with $\bar{v}(0)=v_{0}$. We refer to (2.24) as the reduced 3D Navier-Stokes evolutionary equation. Note that $\bar{v}=\left(\bar{v}_{1}, \bar{v}_{2}, \bar{v}_{3}\right)$ is a three dimensional vector field on $Q_{3}$, and $\bar{v}$ does not depend on $x_{3}$.

The reduced 3D Navier-Stokes evolutionary equation incorporates the 2DNS on $Q_{2}$. In order to see this, we let $L^{2}\left(Q_{2}, \mathbb{R}^{2}\right)$ denote the $L^{2}$ space of 2dimensional vector fields $m=\left(m_{1}, m_{2}\right)$ which depend on $\left(x_{1}, x_{2}\right) \in Q_{2}$. Let $\mathbb{P}_{2}$ denote the orthogonal projection of $L^{2}\left(Q_{2}, \mathbb{R}^{2}\right)$ onto $H\left(Q_{2}\right)$, where $H\left(Q_{2}\right)$ is the closure in $L^{2}\left(Q_{2}, \mathbb{R}^{2}\right)$ of those smooth functions $m$ that are periodic on $Q_{2}$ and satisfy $\int_{Q_{2}} m d x=0$ and $\left(D_{1} m_{1}+D_{2} m_{2}\right)=0$. One then has

$$
\mathbb{P}_{\varepsilon}\left(\begin{array}{c}
\left(\bar{v}_{1} D_{1}+\bar{v}_{2} D_{2}\right) \bar{v}_{1} \\
\left(\bar{v}_{1} D_{1}+\bar{v}_{2} D_{2}\right) \bar{v}_{2} \\
\left(\bar{v}_{1} D_{1}+\bar{v}_{2} D_{2}\right) \bar{v}_{3}
\end{array}\right)=\left(\begin{array}{c}
\left.\mathbb{P}_{2}\left(\begin{array}{c}
\left(\bar{v}_{1} D_{1}+\bar{v}_{2} D_{2}\right) \bar{v}_{1} \\
\left(\bar{v}_{1} D_{1}+\bar{v}_{2} D_{2}\right) \bar{v}_{2}
\end{array}\right)\right) \\
\left(\bar{v}_{1} D_{1}+\bar{v}_{2} D_{2}\right) \bar{v}_{3}
\end{array}\right)
$$

and

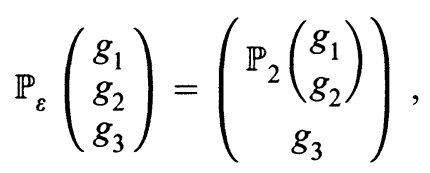

where $g=\left(g_{1}, g_{2}, g_{3}\right) \in M L^{2}\left(Q_{2}, \mathbb{R}^{3}\right)$. Furthermore, $\bar{v}$ is a solution of the reduced 3D Navier-Stokes evolutionary equation (2.24) if and only if $m=$ $\left(\bar{v}_{1}, \bar{v}_{2}\right)$ is a solution of the 2D Navier-Stokes evolutionary equation

$$
\frac{d}{d t} m-\nu\left(D_{1}^{2}+D_{2}^{2}\right) m+\mathbb{P}_{2}(m \cdot \nabla) m=\left(g_{1}, g_{2}\right),
$$

and $\bar{v}_{3}$ is a solution of the linear equation

$$
\frac{d}{d t} \bar{v}_{3}-\nu\left(D_{1}^{2}+D_{2}^{2}\right) \bar{v}_{3}+\left(\bar{v}_{1} D_{1}+\bar{v}_{2} D_{2}\right) \bar{v}_{3}=g_{3},
$$

where $g=\left(g_{1}, g_{2}, g_{3}\right)=M \mathbb{P}_{\varepsilon} f$.

If we want to emphasize that the terms in (2.24) do not depend on $\varepsilon$ or $x_{3}$, we introduce the following notation. For $i=1,2,3$ we set $V_{0}^{i}=M V_{\varepsilon}^{i}$, $H_{0}=V_{0}^{0}=M H_{\varepsilon}\left(Q_{3}\right)$. We denote by $A_{0}$ the restriction of $A_{\varepsilon}$ to $V_{0}^{2}$. If $\bar{v}$ is in $V_{0}^{2}$, then $A_{0} \bar{v}=-\left(\partial^{2} / \partial x_{1}^{2}+\partial^{2} / \partial x_{2}^{2}\right) \bar{v}$. We also set $B_{0}(\bar{v}, \bar{v})=\mathbb{P}_{\varepsilon}(\bar{v} \cdot \nabla) \bar{v}$ if $\bar{v}$ is in $V_{0}^{1}$. Note that $B_{0}(\bar{v}, \bar{v})=B_{\varepsilon}(\bar{v}, \bar{v})$. The reduced 3D Navier-Stokes evolutionary equation (2.24) now becomes

$$
\bar{v}^{\prime}+\nu A_{0} \bar{v}+B_{0}(\bar{v}, \bar{v})=g
$$

with $\bar{v}(0)=v_{0}$ in $V_{0}^{1}$ and $g=M \mathbb{P}_{\varepsilon} f$.

2.7. The trilinear form $b_{\varepsilon}$. The trilinear form $b_{\varepsilon}(u, v, w)$ is defined by

$$
b_{\varepsilon}(u, v, w)=\sum_{i, j=1}^{3} \int_{Q_{3}} \varepsilon^{-\{i\}} u_{i}\left(D_{i} v_{j}\right) w_{j} d x,
$$


provided the integrals are all defined, where $\{1\}=\{2\}=0$ and $\{3\}=1$. If $u, v, w \in V_{\varepsilon}^{1}$, then (2.25) is well defined, and since $\mathbb{P}_{\varepsilon}$ is an orthogonal projection with $\mathbb{P}_{\varepsilon} w=w$, one has

$$
\begin{aligned}
\left\langle B_{\varepsilon}(u, v), w\right\rangle & =\left\langle\mathbb{P}_{\varepsilon}\left(u \cdot \nabla_{\varepsilon}\right) v, w\right\rangle=\left\langle\left(u \cdot \nabla_{\varepsilon}\right) v, \mathbb{P}_{\varepsilon} w\right\rangle \\
& =\left\langle\left(u \cdot \nabla_{\varepsilon}\right) v, w\right\rangle=b_{\varepsilon}(u, v, w) .
\end{aligned}
$$

Note that

$$
b_{\varepsilon}\left(v^{1}, w, v^{2}\right)=-b_{\varepsilon}\left(v^{1}, v^{2}, w\right)=0, \quad b_{\varepsilon}\left(w, v^{1}, v^{2}\right)=0,
$$

whenever $M v^{i}=v^{i}, i=1,2$, and $M w=0$.

2.8. Statement of regularity theorems. Theorem $A$, which is stated in $\S 1$, gives a sufficient condition for the nonlinear evolutionary equation (2.5) to have a strong solution $u(t)$ that remains in $V_{\varepsilon}^{1}$ for all $t \geq 0$ and in $V_{\varepsilon}^{2}$ for all $t>0$. In a moment we shall define the sets $\mathscr{R}(\varepsilon)$ and $\mathscr{S}(\varepsilon)$, and in $\S 2.10$ we explain why these are large sets. The key to this is the following Hypothesis $\mathrm{H}(\mathrm{a}, \mathrm{b})$ :

We shall say that the bounded monotone functions $\eta_{i}(\varepsilon)$ defined for $0<\varepsilon \leq$ $1, i=1,2,3,4$, and constants $r$ and $p$ satisfy Hypothesis $\mathbf{H}(\mathbf{a}, \mathbf{b})$, where $a$ and $b$ are positive, provided:

(1) $p \geq-1, r>-2$.

(2) $\varepsilon^{1 / 4} \eta_{i}^{-1} \rightarrow 0$ as $\varepsilon \rightarrow 0, i=1,2$.

(3) $\varepsilon^{1 / 8} \eta_{i}^{-1} \rightarrow 0$ as $\varepsilon \rightarrow 0, i=3,4$.

(4) $\varepsilon^{1 / 4} Q(\varepsilon)$ is bounded for $0<\varepsilon \leq 1$, where

$$
Q(\varepsilon)=\left|\log \left(2 C_{5}^{2} \nu^{-2} \varepsilon^{2+r-p} \eta_{4}^{-2} \eta_{3}^{2}\right)\right|
$$

(5) Let $a>0$ be fixed. Then one has

$$
\left\{\begin{array}{l}
\varepsilon^{5 / 8} \eta^{-2} \exp \left(a \eta^{-4}\right) \rightarrow 0 \\
\eta^{-2} \rightarrow \infty
\end{array}\right.
$$

as $\varepsilon \rightarrow 0^{+}$, where

$$
\eta^{-2} \stackrel{\text { def }}{=} \max \left(4 \eta_{1}^{-2}+k_{1}^{2} \eta_{3}^{-4}+k_{2}^{2} \varepsilon^{2+r} \eta_{4}^{-2}, 1\right)
$$

and $\varepsilon^{5 / 8} \exp \left(2 a \eta_{2}^{-4}\right)$ is bounded for $0<\varepsilon \leq 1$. (The constants $k_{1}$ and $k_{2}$ are defined in Lemma 3.1.)

(6) Let $b>0$ be fixed. Then for any $\lambda, 0<\lambda<1$, there is an $\varepsilon_{4}=$ $\varepsilon_{4}(b, \lambda)>0$ such that one has

$$
\eta_{2}^{-2} \exp \left(b \eta_{2}^{-4}\right) \leq \lambda\left(4 \eta_{1}^{-2}+k_{1}^{2} \eta_{3}^{-4}\right), \quad 0<\varepsilon<\varepsilon_{4} .
$$

(7) The function $\varepsilon^{4+2 r} \eta_{4}^{-4}\left(\log \eta^{-4}+1\right)$ is bounded as $\varepsilon \rightarrow 0^{+}$.

Remarks. 1. The choice of the function $\eta_{i}$ may depend on the parameters $p, r, a$, and $b$.

2. Here is an example where Hypothesis $H(a, b)$ is satisfied for any given choice of $a>0$ and $b>0$. We begin with statement (5). If $\eta$ is given by (2.28), then

$$
1 \leq \eta^{-4} \leq\left(48 \eta_{1}^{-4}+3 k_{1}^{4} \eta_{3}^{-8}+3 k_{2}^{4} \varepsilon^{2(2+r)} \eta_{4}^{-4}\right) .
$$


Next fix $p \geq-1$ and set

$$
\left\{\begin{array}{l}
r=-2+\delta, \quad \delta>0 \\
\eta_{4}^{-1}=-\log \varepsilon ; \\
\eta_{3}^{-2}=\eta_{1}^{-1}
\end{array}\right.
$$

If $\eta_{1}^{-2}=\left(-\log \varepsilon^{\alpha}\right)^{1 / 2}$, where $\alpha>0$, then conditions (1), (2), and (3) hold. Furthermore (4) and (7) are valid. In addition, one has

$$
\eta^{-4} \leq\left(48+3 k_{1}^{4}\right)\left(-\log \varepsilon^{\alpha}\right)+3 k_{2}^{4} \varepsilon^{2(2+r)}(-\log \varepsilon)^{4} .
$$

Consequently (2.27) is valid provided

$$
\varepsilon^{5 / 8}\left(-\log \varepsilon^{\alpha}\right)^{1 / 2} \varepsilon^{-a\left(48+3 k_{1}^{4}\right) \alpha} \rightarrow 0 \quad \text { as } \varepsilon \rightarrow 0^{+} .
$$

In other words, if $\alpha$ is chosen such that $\frac{5}{8}>\left(48+3 k_{1}^{4}\right) a \alpha$, then (2.27) is satisfied. Likewise, by choosing $\eta_{2}^{-2}$ so that

$$
\eta_{2}^{-2}=\left(\log \left(-\log \varepsilon^{\alpha}\right)^{\beta}\right)^{1 / 2}, \quad \text { where } \beta>0 \text { and } 2 b \beta<1,
$$

we see that statement $(6)$ is valid. $\left(\eta_{2}^{-2}=\log (\log (-\log \varepsilon))\right.$ is another possible choice of $\eta_{2}^{-2}$.) Also note that this example satisfies condition (2.55) below, provided $-1 \leq p<0$.

3. Another example of interest occurs when $\eta_{2}$ and $\eta_{4}$ are positive constants and $\eta_{1}$ and $\eta_{3}$ are as in Remark 2. This situation arises in the study of the semicontinuity of the attractors, see $\S 6$.

In order to prove Theorem A we shall analyze the dilated Navier-Stokes evolutionary equation on $Q_{3}$. This analysis consists of two major steps. The first step, which we call the $H^{1}$-Regularity Theorem, is to show that there is a constant $K_{1}$ such that $\left\|A_{\varepsilon}^{1 / 2} u(t)\right\|^{2} \leq K_{1}^{2}$ for all $t \geq 0$ and that there is a constant $L_{5}$, which does not depend on the initial data, such that $\lim _{\sup } \operatorname{si\infty }_{t \rightarrow A_{\varepsilon}} u(t) \|^{2}$ $\leq L_{5}^{2}$. The second step, which we call the $H^{2}$-Regularity Theorem, is to show that $u(t) \in D\left(A_{\varepsilon}\right)$ for all $t>0$ and that there is a constant $L_{6}$, which does not depend on the initial data, such that $\limsup _{t \rightarrow \infty}\left\|A_{\varepsilon} u(t)\right\|^{2} \leq L_{6}^{2}$.

Theorem 1. $H^{1}$-Regularity. Let $\eta_{i}, i=1,2,3,4, r$, and $p$ satisfying $H y$ pothesis $H(a, b)$, where $a$ and $b$ are sufficiently large. Then there exist $\varepsilon_{0}>0$, $k_{2}>0$, a continuous function $\Gamma \in C([0, \infty), \mathbb{R})$, and for all $\varepsilon, 0<\varepsilon \leq \varepsilon_{0}, a$ time $\widehat{T}_{1}=\widehat{T}_{1}(\varepsilon)>0$ such that for all $\varepsilon, 0<\varepsilon \leq \varepsilon_{0}$, whenever $u_{0} \in D\left(A_{\varepsilon}^{1 / 2}\right)$ and $f \in L^{\infty}\left((0, \infty), L^{2}\left(Q_{3}\right)\right)$ satisfy

$$
\begin{cases}\left\|A_{\varepsilon}^{1 / 2} v_{0}\right\|^{2} \leq \eta_{1}^{-2}, & \left\|M \mathbb{P}_{\varepsilon} f\right\|_{\infty}^{2} \leq \eta_{2}^{-2}, \\ \left\|A_{\varepsilon}^{1 / 2} w_{0}\right\|^{2} \leq \varepsilon^{p} \eta_{3}^{-2}, & \left\|(I-M) \mathbb{P}_{\varepsilon} f\right\|_{\infty}^{2} \leq \varepsilon^{r} \eta_{4}^{-2},\end{cases}
$$

then (2.5) has a solution $u$ that belongs to $C^{0}\left([0, \infty), V_{\varepsilon}^{1}\right) \cap L^{\infty}\left((0, \infty), V_{\varepsilon}^{1}\right)$, i.e., one has

$$
\left\|A_{\varepsilon}^{1 / 2} u(t)\right\|^{2} \leq K_{1}^{2}, \quad t \geq 0
$$


where $K_{1}$ depends on $\nu, \lambda_{1}$, and $\eta_{i}, i=1,2,3,4$, but not on $t \geq 0$. Moreover, the components of $u=v+w$ satisfy

$$
\left\|A_{\varepsilon}^{1 / 2} v(t)\right\|^{2} \leq \Gamma\left(\eta_{2}^{-2}\right), \quad t \geq \widehat{T}_{1},
$$

where $\Gamma$ is given by (3.84), and

$$
\left\|A_{\varepsilon}^{1 / 2} w(t)\right\|^{2} \leq \max \left(\varepsilon^{2}, k_{2}^{2} \varepsilon^{2+r} \eta_{4}^{-2}\right), \quad t \geq \widehat{T}_{1} .
$$

Remarks. 1. The principal objective in any study of the global regularity of solutions of the 3DNS is to show that the conclusions of Theorem 1 apply for all $u_{0} \in V_{\varepsilon}^{1}$ and $\mathbf{P}_{\varepsilon} f \in L^{\infty}\left((0, \infty), L^{2}\left(Q_{3}\right)\right)$. Since the techniques developed in this paper seem to fall short of achieving this goal, we seek, instead, to find the largest possible $u_{0}$ and $f$ (see (2.29)) for which we can prove global regularity.

2. It follows from (2.21), (2.31), and (2.32) that

$$
\left\|A_{\varepsilon}^{1 / 2} u(t)\right\|^{2} \leq L_{5}^{2}, \quad t \geq \widehat{T}_{1}
$$

where $L_{5}^{2}=\Gamma\left(\eta_{2}^{-2}\right)+\max \left(\varepsilon^{2}, k_{2}^{2} \varepsilon^{2+r} \eta_{4}^{-2}\right)$. Since $L_{5}^{2}$ does not depend on $\eta_{1}$, $\eta_{3}$, or $p$, it is independent of the initial condition $u_{0}$. Furthermore, if $\eta_{2}^{-2}$ and $\varepsilon^{2+r} \eta_{4}^{-2}$ are bounded for $0<\varepsilon \leq 1$, it follows from Theorem 1 that $L_{5}^{2}$ can be chosen independent of $\varepsilon$.

Theorem 2. $H^{2}$-Regularity. Let $r, p$, and $\eta_{i}, i=1,2,3,4$, satisfy Hypothesis $H(a, b)$, where $a$ and $b$ are sufficiently large. If $u_{0} \in V_{\varepsilon}^{1}$ and

$$
\mathbb{P}_{\varepsilon} f \in C^{0}\left([0, \infty), H_{\varepsilon}\right) \cap L^{\infty}\left((0, \infty), H_{\varepsilon}\right) \cap W^{1, \infty}\left((0, \infty), D\left(A_{\varepsilon}^{-1 / 2}\right)\right)
$$

satisfy (2.29), then for $0<\varepsilon \leq \varepsilon_{0}$, where $\varepsilon_{0}$ is given by Theorem 1 , the solution $u(t)$ of $(2.5)$ belongs to $C^{0}\left((0, \infty), V_{\varepsilon}^{2}\right)$. Furthermore there exist three positive constants $K_{2}, K_{3}$ and $K_{4}$, which depend on $\nu, \lambda_{1}, \eta_{i}, i=1,2,3,4$, and $K_{1}$, where $K_{1}$ is given by (2.30), such that

$$
\begin{cases}\left\|A_{\varepsilon} u(t)\right\|^{2} \leq K_{2}^{2}+K_{3}^{2}\left\|A_{\varepsilon}^{-1 / 2} \mathbb{P}_{\varepsilon} f^{\prime}\right\|_{\infty}^{2}+K_{4}^{2} t^{-1} & \text { for } 0<t \leq 1, \\ \left\|A_{\varepsilon} u(t)\right\|^{2} \leq K_{2}^{2}+K_{3}^{2}\left\|A_{\varepsilon}^{-1 / 2} \mathbb{P}_{\varepsilon} f^{\prime}\right\|_{\infty}^{2} & \text { for } t \geq 1 .\end{cases}
$$

Moreover, there is a positive continuous function $\Gamma_{2}$ on $[0, \infty)$ given by (4.22), such that

$$
\left\|A_{\varepsilon} u(t)\right\|^{2} \leq L_{6}^{2}, \quad t \geq \widehat{T}_{1}+1
$$

where $\widehat{T}_{1}$ is given by Theorem $1, L_{6}^{2}=\Gamma_{2}\left(L_{5}^{2}\right)$, and $L_{5}^{2}$ is given in (2.33).

If, in addition, $u_{0}$ belongs to $D\left(A_{\varepsilon}\right)$, then the solution $u$ of $(2.5)$ belongs to the space $\left.C^{0}([0, \infty)), V_{\varepsilon}^{2}\right)$, and one has

$$
\left\|A_{\varepsilon} u(t)\right\|^{2} \leq K_{5}^{2}+K_{6}^{2}\left\|A_{\varepsilon} u_{0}\right\|^{2}+K_{7}^{2}\left\|A_{\varepsilon}^{-1 / 2} \mathbb{P}_{\varepsilon} f^{\prime}\right\|_{\infty}^{2}, \quad 0 \leq t \leq 1,
$$

where $K_{5}, K_{6}$, and $K_{7}$ are positive constants depending on $\nu, \lambda_{1}, \eta_{i}, i=$ $1,2,3,4$, and $K_{1}$. 
Let $\mathscr{B}_{\varepsilon}^{0}, \mathscr{B}_{\varepsilon}{ }^{1}$, and $\mathscr{B}_{\varepsilon}{ }^{2}$ denote the following bounded sets in $V_{\varepsilon}^{1}=D\left(A_{\varepsilon}^{1 / 2}\right)$ :

$$
\begin{aligned}
& \mathscr{B}_{\varepsilon} \stackrel{\text { def }}{=}\left\{u=v+w:\left\|A_{\varepsilon}^{1 / 2} v\right\|^{2} \leq 4 \eta_{1}^{-2}+k_{1}^{2} \eta_{3}^{-4},\left\|A_{\varepsilon}^{1 / 2} w\right\|^{2} \leq k_{2}^{2} \varepsilon^{2+r} \eta_{4}^{-2}\right\}, \\
& \mathscr{B}_{\varepsilon}^{2} \stackrel{\text { def }}{=} \bigcup_{t \geq 0} S_{\varepsilon}\left(\mathbb{P}_{\varepsilon} f, t\right)\left(\mathscr{B}_{\varepsilon}^{0} \cup \mathscr{B}_{\varepsilon}^{1}\right) \text {, }
\end{aligned}
$$

where $u(t)=S_{\varepsilon}\left(\mathbb{P}_{\varepsilon} f, t\right) u_{0}$ is the strong solution of the equation (2.5) with initial data $u_{0}$. Due to the Lemmas 3.1 and $3.2, \mathscr{B}_{\varepsilon}^{2}$ is well defined and is a bounded set in $V_{\varepsilon}^{1}$.

Remarks. 1. Since $L_{5}^{2}$ does not depend on the initial condition $u_{0}$, it follows from Theorem 2 that the bound $L_{6}^{2}$ does not depend on $u_{0}$. Furthermore, if $f$ is chosen so that $\left\|\mathbb{P}_{\varepsilon} f\right\|_{\infty}$ and $\left\|A_{\varepsilon}^{-1 / 2} \mathbb{P}_{\varepsilon} f^{\prime}\right\|_{\infty}$ are bounded for $0<\varepsilon \leq 1$, then $L_{6}^{2}$ can be chosen to be independent of $\varepsilon$.

2. One can obtain other $H^{2}$-regularity results if one assumes that $f$ has more spatial regularity, e.g., $\mathbb{P}_{\varepsilon} f \in L^{\infty}\left((0, \infty) ; V_{\varepsilon}^{1}\right)$ instead of satisfying (2.34). (See Foiaş, Guillopé, and Temam (1981).)

3. If in addition to the hypotheses of Theorem 2 , the function $f$ belongs to $W^{1, \infty}\left((0, \infty), H_{\varepsilon}\right)$, then from (2.6) and (2.22) one finds that

$$
\left\|A_{\varepsilon}^{-1 / 2} \mathbb{P}_{\varepsilon} f^{\prime}\right\|_{\infty}^{2} \leq \lambda_{1}^{-1}\left\|M \mathbb{P}_{\varepsilon} f^{\prime}\right\|_{\infty}^{2}+C_{5}^{2} \varepsilon^{2}\left\|(I-M) \mathbb{P}_{\varepsilon} f^{\prime}\right\|_{\infty}^{2},
$$

which can be used in (2.35) (2.36), and (2.37).

2.9. Small data regularity. As mentioned in the introduction, it is known that the 3DNS has a globally regular solution whenever the data of the problem are small. The global regularity with small data, which is valid for any reasonable bounded 3-dimensional region, is a simple consequence of the Stable Manifold Theorem. We emphasize that our theorems, which are valid for thin 3-dimensional regions, are not consequences of the small data arguments. Before showing this though, it will be useful to recall one of these small data arguments at this time. ${ }^{2}$

The argument we give here will be for the Navier-Stokes evolutionary equation (2.14) on an arbitrary bounded region $\Omega$ in $\mathbb{R}^{3}$. We will not exploit, at this time, the assumption that $\Omega=\Omega_{\varepsilon}$ is a thin domain, an assumption which is of special interest elsewhere in this paper. We assume here, for simplicity, that $F \in L^{2}(\Omega)$ does not depend on time.

We will use the standard $3 \mathrm{D}$ estimate for $B\left(U^{1}, U^{2}\right)$ :

$$
\left|\left\langle B\left(U^{1}, U^{2}\right), U^{3}\right\rangle\right| \leq C_{8}\left\|A^{1 / 2} U^{1}\right\|_{L^{2}(\Omega)}\left\|A^{1 / 2} U^{2}\right\|_{L^{2}(\Omega)}^{1 / 2}\left\|A U^{2}\right\|_{L^{2}(\Omega)}^{1 / 2}\left\|U^{3}\right\|_{L^{2}(\Omega)} ;
$$

see Temam $(1977,1983)$ or Constantin and Foiaş $(1988)$. The constant $C_{8}=$ $C_{8}(\Omega)$ depends on $\Omega$.

\footnotetext{
${ }^{2}$ There are several approaches to proving the global regularity of solutions with small data. For all practical purposes, these arguments all lead to the same conclusions described here.
} 
By taking the scalar product of (2.14) with $A U$ we find that

$$
\begin{aligned}
\frac{1}{2} \frac{d}{d t} & \left\|A^{1 / 2} U\right\|_{L^{2}(\Omega)}^{2}+\nu\|A U\|_{L^{2}(\Omega)}^{2} \\
& \leq\left\|\mathbb{P}_{3} F\right\|_{L^{2}(\Omega)}\|A U\|_{L^{2}(\Omega)}+C_{8}\left\|A^{1 / 2} U\right\|_{L^{2}(\Omega)}^{3 / 2}\|A U\|_{L^{2}(\Omega)}^{3 / 2} \\
& \leq \frac{\nu}{2}\|A U\|_{L^{2}(\Omega)}^{2}+\frac{1}{\nu}\left\|\mathbb{P}_{3} F\right\|_{L^{2}(\Omega)}^{2}+\frac{27}{4 \nu^{3}} C_{8}^{4}\left\|A^{1 / 2} U\right\|_{L^{2}(\Omega)}^{6}
\end{aligned}
$$

We then get

$$
\frac{d}{d t}\left\|A^{1 / 2} U\right\|_{L^{2}(\Omega)}^{2}+\nu\|A U\|_{L^{2}(\Omega)}^{2} \leq \frac{2}{\nu}\left\|\mathbb{P}_{3} F\right\|_{L^{2}(\Omega)}^{2}+\frac{27}{2 \nu^{3}} C_{8}^{4}\left\|A^{1 / 2} U\right\|_{L^{2}(\Omega)}^{6},
$$

which in turn implies that

$$
\frac{d}{d t}\left\|A^{1 / 2} U\right\|_{L^{2}(\Omega)}^{2}+\lambda_{1} \nu\left\|A^{1 / 2} U\right\|_{L^{2}(\Omega)}^{2} \leq \frac{2}{\nu}\left\|\mathbb{P}_{3} F\right\|_{L^{2}(\Omega)}^{2}+\frac{27}{2 \nu^{3}} C_{8}^{4}\left\|A^{1 / 2} U\right\|_{L^{2}(\Omega)}^{6} .
$$

Now set $R_{0}^{2}=\left\|A^{1 / 2} U_{0}\right\|_{L^{2}(\Omega)}^{2}+\left\|\mathbb{P}_{3} F\right\|_{L^{2}(\Omega)}^{2}$ and $N>\max \left(1,4 \lambda_{1}^{-1} \nu^{-2}\right)$. Since $R_{0}^{2} \geq\left\|A^{1 / 2} U_{0}\right\|_{L^{2}(\Omega)}^{2}$ and $N>1$, it follows from Lemma 3.0 below that there is a $T^{N}, 0<T^{N} \leq \infty$, such that

$$
\left\|A^{1 / 2} U(t)\right\|_{L^{2}(\Omega)}^{2} \leq N R_{0}^{2}, \quad 0 \leq t<T^{N} .
$$

We assume, without loss of generality, that $\left[0, T^{N}\right)$ is the maximal time interval for which (2.43) is valid.

Next assume that

$$
\frac{27 C_{8}^{4}}{2 \nu^{3}} N^{2} R_{0}^{4} \leq \frac{\lambda_{1} \nu}{2}
$$

Inequality $(2.44)$ is the precise assumption that the data for (2.14) are small. Because of (2.43) and (2.44), it follows from (2.42) that

$$
\frac{d}{d t}\left\|A^{1 / 2} U\right\|_{L^{2}(\Omega)}^{2}+\frac{\lambda_{1} \nu}{2}\left\|A^{1 / 2} U\right\|_{L^{2}(\Omega)}^{2} \leq \frac{2}{\nu}\left\|\mathbb{P}_{3} F\right\|_{L^{2}(\Omega)}^{2}
$$

By applying the Gronwall inequality to $(2.45)$ we get

$$
\left\|A^{1 / 2} U(t)\right\|_{L^{2}(\Omega)}^{2} \leq \exp \left(-\frac{\lambda_{1} \nu}{2} t\right)\left\|A^{1 / 2} U_{0}\right\|_{L^{2}(\Omega)}^{2}+\frac{4}{\lambda_{1} \nu^{2}}\left\|\mathbb{P}_{3} F\right\|_{L^{2}(\Omega)}^{2}<N R_{0}^{2}
$$

for $0<t \leq T^{N}$. Consequently (2.10) implies that $T^{N}=\infty$, which completes the proof of global regularity of solutions for small data.

Remark. In the case of a thin domain $\Omega_{\varepsilon}$, one has $C_{8}=C \varepsilon^{-1 / 2}$, where $C$ is independent of $\varepsilon$. As a result, inequality (2.44) can be rewritten as

$$
\left\|A^{1 / 2} U_{0}\right\|_{L^{2}\left(\Omega_{\varepsilon}\right)}^{2}+\left\|\mathbb{P}_{3} F\right\|_{L^{2}\left(\Omega_{\varepsilon}\right)}^{2} \leq C^{*} \varepsilon
$$

where $C^{*}$ depends on $\nu$ and $\lambda_{1}$ but not on $\varepsilon$. 
2.10. Large data regularity. We now show that the inequalities (2.29) describe large data conditions on both $Q_{3}$ and the thin domain $\Omega_{\varepsilon}$. The inequalities (2.29) describe the norms of the data for (2.5) in the space $L^{2}\left(Q_{3}\right)$. We now set $p=r=-1$ and assume that $\eta_{i}(\varepsilon) \rightarrow 0$ as $\varepsilon \rightarrow 0^{+}$for $1 \leq i \leq 4$. By using the mapping $J_{\varepsilon}$ together with (2.3), (2.15), and (2.21) one finds that

$$
\begin{aligned}
\left\|A^{1 / 2} U_{0}\right\|_{L^{2}\left(\Omega_{\varepsilon}\right)}^{2} & =\left\|A^{1 / 2} V_{0}\right\|_{L^{2}\left(\Omega_{\varepsilon}\right)}^{2}+\left\|A^{1 / 2} W_{0}\right\|_{L^{2}\left(\Omega_{\varepsilon}\right)}^{2} \\
& =\varepsilon\left\|A_{\varepsilon}^{1 / 2} v_{0}\right\|_{L^{2}\left(Q_{3}\right)}^{2}+\varepsilon\left\|A_{\varepsilon}^{1 / 2} w_{0}\right\|_{L^{2}\left(Q_{3}\right)}^{2} \\
& \leq \varepsilon \eta_{1}^{-2}+\eta_{3}^{-2} .
\end{aligned}
$$

Similarly one has

$$
\left\|\mathbb{P}_{3} F\right\|_{L^{2}\left(\Omega_{\varepsilon}\right)}^{2}=\varepsilon\left\|\mathbb{P}_{\varepsilon} f\right\|_{L^{2}\left(Q_{3}\right)}^{2} \leq \varepsilon \eta_{2}^{-2}+\eta_{4}^{-2} .
$$

Inequalities (2.47) and (2.48) imply that

$$
\left\|A^{1 / 2} U_{0}\right\|_{L^{2}\left(\Omega_{\varepsilon}\right)}^{2}+\left\|\mathbb{P}_{3} F\right\|_{L^{2}\left(\Omega_{\varepsilon}\right)}^{2} \leq \varepsilon\left(\eta_{1}^{-2}+\eta_{2}^{-2}\right)+\eta_{3}^{-2}+\eta_{4}^{-2} .
$$

Assume that we choose $\eta_{3}(\varepsilon)=\eta_{4}(\varepsilon)=1$. Then, even in this case, condition (2.49) is much weaker than condition (2.46) since we allow $\eta_{1}^{-2}(\varepsilon)$ and $\eta_{2}^{-2}(\varepsilon)$ to go to $\infty$ as $\varepsilon \rightarrow 0^{+}$. To put it another way, assume that $F$ satisfies $(2.46)$ and really depends on the three variables $\left(y_{1}, y_{2}, y_{3}\right)$, and let $U_{1}=U_{1}\left(y_{1}, y_{2}\right)$ satisfy $U_{1} \in H^{2}\left(Q_{2}, \mathbb{R}^{3}\right)$, with periodic boundary conditions, divergence free, and $\left\|U_{1}\right\|_{L^{2}\left(Q_{2}\right)}=1$. Then

$$
U_{0}\left(y_{1}, y_{2}, y_{3}\right)=\eta_{1}^{-1}(\varepsilon) U_{1}\left(y_{1}, y_{2}\right)
$$

will satisfy $(2.49)$, but not $(2.46)$, for small $\varepsilon$, whenever $\eta_{1}^{-1}(\varepsilon) \rightarrow \infty$ as $\varepsilon \rightarrow$ $0^{+}$.

For $0<\varepsilon \leq \varepsilon_{0}$ we define $R_{1}(\varepsilon)$ to be the collection of $v_{0} \in M V_{\varepsilon}^{1}$ such that

$$
\left\|A_{\varepsilon}^{1 / 2} v_{0}\right\|_{L^{2}\left(Q_{3}\right)}^{2}=\left\|v_{0}\right\|_{V_{\varepsilon}^{1}}^{2} \leq \eta_{1}^{-2}
$$

and $R_{2}(\varepsilon)$ to be the collection of $w_{0} \in(I-M) V_{\varepsilon}^{1}$ such that

$$
\left\|A_{\varepsilon}^{1 / 2} w_{0}\right\|_{L^{2}\left(Q_{3}\right)}^{2}=\left\|w_{0}\right\|_{V_{\varepsilon}^{1}}^{2} \leq \varepsilon^{-1} \eta_{3}^{-2} \text {. }
$$

Set $R(\varepsilon)=R_{1}(\varepsilon)+R_{2}(\varepsilon)$, and let $\mathscr{R}_{1}(\varepsilon)=J_{\varepsilon} R_{1}(\varepsilon), \mathscr{R}_{2}(\varepsilon)=J_{\varepsilon} R_{2}(\varepsilon)$, and $\mathscr{R}(\varepsilon)=J_{\varepsilon} R(\varepsilon)$ denote the corresponding sets in $H^{1}\left(\Omega_{\varepsilon}\right)$. The sets $R_{1}(\varepsilon)$ and $R_{2}(\varepsilon)$ are bounded sets in $M V_{\varepsilon}^{1}$ and $(I-M) V_{\varepsilon}^{1}$ with $V_{\varepsilon}^{1}$-radius being $\eta_{1}^{-1}$ and $\varepsilon^{-1 / 2} \eta_{3}^{-1}$, respectively. From (2.3) we see that $V_{0}=J_{\varepsilon} v_{0} \in \mathscr{R}_{1}(\varepsilon)$ and $W_{0}=J_{\varepsilon} w_{0} \in \mathscr{R}_{2}(\varepsilon)$ if and only if

$$
\left\|A^{1 / 2} V_{0}\right\|_{L^{2}\left(\Omega_{\varepsilon}\right)}^{2} \leq \varepsilon \eta_{1}^{-2} \text { and }\left\|A^{1 / 2} W_{0}\right\|_{L^{2}\left(\Omega_{\varepsilon}\right)}^{2} \leq \eta_{3}^{-2}
$$

Thus $\mathscr{R}_{1}(\varepsilon)$ and $\mathscr{R}_{2}(\varepsilon)$ contain bounded sets in $M H^{1}\left(\Omega_{\varepsilon}\right)$ and $(I-M) H^{1}\left(\Omega_{\varepsilon}\right)$ with $H^{1}\left(\Omega_{\varepsilon}\right)$-radius being $C \varepsilon^{1 / 2} \eta_{1}^{-1}$ and $C \eta_{3}^{-1}$, respectively. The example 
constructed in $\S 2.8$ gives information on the size of these radii as $\varepsilon \rightarrow 0^{+}$. The point to note in this example is that $\eta_{3}^{-1}=\left(-\log \varepsilon^{\alpha}\right)^{1 / 8}$. The assertion in Theorem A that $\mathscr{R}(\varepsilon)$ is large is a heuristic formulation of the fact that $\eta_{i}^{-1} \rightarrow \infty$ as $\varepsilon \rightarrow 0^{+}, i=1,3$.

Similarly we define $S(\varepsilon)$ to be the collection of $f \in L^{\infty}\left((0, \infty), L^{2}\left(Q_{3}\right)\right)$ that satisfy

$$
\left\|M \mathbb{P}_{\varepsilon} f(t)\right\|_{L^{2}\left(Q_{3}\right)}^{2} \leq \eta_{2}^{-2} \text { and }\left\|(I-M) \mathbb{P}_{\varepsilon} f(t)\right\|_{L^{2}\left(Q_{3}\right)}^{2} \leq \varepsilon^{r} \eta_{4}^{-2}
$$

for $0<t<\infty$ and set $\mathscr{S}(\varepsilon)=J_{\varepsilon} S(\varepsilon)$, where $r=-2+\delta$, say $0<\delta \leq \frac{1}{2}$. One then has $F=J_{\varepsilon} f \in \mathscr{S}(\varepsilon)$ if and only if

$$
\left\|M \mathbb{P}_{3} F(t)\right\|_{L^{2}\left(\Omega_{\varepsilon}\right)}^{2} \leq \varepsilon \eta_{2}^{-2} \text { and }\left\|(I-M) \mathbb{P}_{3} F(t)\right\|_{L^{2}\left(\Omega_{\varepsilon}\right)}^{2} \leq \varepsilon^{r+1} \eta_{4}^{-2} .
$$

Once again, the assertion that $\mathscr{S}(\varepsilon)$ is large is a heuristic formulation of the fact that $\eta_{i}^{-1} \rightarrow \infty$ as $\varepsilon \rightarrow 0^{+}, i=2,4$. The example in $\S 2.8$ shows that one can choose $\eta_{4}^{-1}=(-\log \varepsilon)$.

If the initial condition $u_{0}$ belongs to $D\left(A_{\varepsilon}\right)$, as in the case of Theorem 2 , then one has $v_{0}, w_{0} \in D\left(A_{\varepsilon}\right)$, and (2.6) and (2.22) imply that

$$
\left\{\begin{array}{l}
\left\|A_{\varepsilon}^{1 / 2} v_{0}\right\|_{L^{2}\left(Q_{3}\right)}^{2} \leq \lambda_{1}^{-1}\left\|A_{\varepsilon} v_{0}\right\|_{L^{2}\left(Q_{3}\right)}^{2}, \\
\left\|A_{\varepsilon}^{1 / 2} w_{0}\right\|_{L^{2}\left(Q_{3}\right)}^{2} \leq C_{5}^{2} \varepsilon^{2}\left\|A_{\varepsilon} w_{0}\right\|_{L^{2}\left(Q_{3}\right)}^{2} .
\end{array}\right.
$$

This means that $u_{0}=v_{0}+w_{0}$ will satisfy (2.29) provided one has

$$
\left\{\begin{array}{l}
\left\|A_{\varepsilon} v_{0}\right\|_{L^{2}\left(Q_{3}\right)}^{2} \leq \lambda_{1} \eta_{1}^{-2}, \\
\left\|A_{\varepsilon} w_{0}\right\|_{L^{2}\left(Q_{3}\right)}^{2} \leq C_{5}^{-2} \varepsilon^{p-2} \eta_{3}^{-2} .
\end{array}\right.
$$

By using the mapping $J_{\varepsilon}$ and (2.3), we see that (2.50) can be written in the equivalent form

$$
\left\{\begin{array}{l}
\left\|A V_{0}\right\|_{L^{2}\left(\Omega_{\varepsilon}\right)}^{2} \leq \lambda_{1} \varepsilon \eta_{1}^{-2}, \\
\left\|A W_{0}\right\|_{L^{2}\left(\Omega_{\varepsilon}\right)}^{2} \leq C_{5}^{-2} \varepsilon^{p-1} \eta_{3}^{-2} .
\end{array}\right.
$$

Thus $\mathscr{R}_{2}(\varepsilon)$ contains a bounded set in $(I-M) H^{2}\left(\Omega_{\varepsilon}\right)$ of $H^{2}\left(\Omega_{\varepsilon}\right)$-radius $\geq$ $C \varepsilon^{p-1}$, where $p \geq 1$.

2.11. Skew-Product dynamics. We will review here some aspects of the theory of skew-product flows in order to describe the (local) attractors for the NavierStokes equations with a time-varying forcing function $f$. We will formulate this general theory for the Navier-Stokes equations on an arbitrary bounded domain $\Omega$ in $\mathbb{R}^{n}$, where $n=2,3$. For simplicity ${ }^{3}$ we will consider forcing functions $f$ in the space

$$
f \in W(\Omega) \stackrel{\text { def }}{=} C^{0}\left(\mathbb{R}, L^{2}(\Omega)\right) \cap L^{\infty}\left(\mathbb{R}, L^{2}(\Omega)\right),
$$

\footnotetext{
${ }^{3}$ By using other topologies one can describe attractors when the forcing function $f$ is discontinuous. See Miller and Sell (1970) or Sacker and Sell (1977) for details.
} 
where $L^{2}(\Omega)=L^{2}\left(\Omega, \mathbb{R}^{n}\right)$. For any linear operator $T$ on $L^{2}(\Omega)$ we let

$$
T W(\Omega)=C^{0}\left(\mathbb{R}, T L^{2}(\Omega)\right) \cap L^{\infty}\left(\mathbb{R}, T L^{2}(\Omega)\right) .
$$

A metrizable topology is introduced in the space $W(\Omega)$ by defining sequential convergence $f_{n} \rightarrow f$ to mean that for any compact set $K \subset \mathbb{R}$ one has

$$
\sup _{t \in K}\left\|f_{n}(t)-f(t)\right\|_{L^{2}(\Omega)} \rightarrow 0 \quad \text { as } n \rightarrow \infty .
$$

We will denote the associated metric by $\operatorname{dist}_{W(\Omega)}$.

For any $f$ satisfying $(2.51)$ and any $\tau \in \mathbb{R}$ we define the translate $f_{\tau}(t) \stackrel{\text { def }}{=}$ $f(\tau+t)$. Note that $f_{\tau} \in W(\Omega)$, and the mapping $(f, \tau) \rightarrow f_{\tau}$ is a continuous mapping of $W(\Omega) \times \mathbb{R}$ onto itself. This means that $f_{\tau}$ defines a (two-sided) flow on $W(\Omega)$. For each $f$ satisfying (2.51) we define the positive hull $\mathrm{H}^{+}(f)$ as

$$
H^{+}(f)=\text { Closure }_{W(\Omega)}\left\{f_{\tau}: \tau \geq 0\right\}
$$

and the hull $H(f)$ as

$$
H(f)=\text { Closure }_{W(\Omega)}\left\{f_{\tau}: \tau \in \mathbb{R}\right\} .
$$

If $f \in W(\Omega)$, then $H^{+}(f)$ and $H(f)$ lie in $W(\Omega)$. The omega-limit set $\omega(f)$ is defined by

$$
\omega(f)=\bigcap_{\tau \geq 0} H^{+}\left(f_{\tau}\right)
$$

Note that $\omega(f)$ is an invariant set in the translational flow on $W(\Omega)$.

Without further assumptions on the forcing function $f$, the omega-limit set $\omega(f)$ can be empty. However, if $H^{+}(f)$ is a compact set, then so is $H^{+}\left(f_{\tau}\right)$ for every $\tau \geq 0$. Since $H^{+}\left(f_{\tau}\right) \subset H^{+}(f)$ for $\tau \geq 0$, we see that if $H^{+}(f)$ is compact, then the omega-limit set $\omega(f)$ is nonempty and compact.

The question of the compactness of $\mathrm{H}^{+}(f)$ can be resolved by using the Ascoli-Arzelá Theorem; see Sell (1967a, 1967b). In particular, if there is a compact set $\mathscr{K} \subseteq L^{2}(\Omega)$ such that $f(t) \in \mathscr{K}$ for all $t \geq 0$ and the mapping $t \rightarrow f(t)$ is a uniformly continuous mapping of $[0, \infty)$ into $L^{2}(\Omega)$, then $H^{+}(f)$ is a nonempty compact set; furthermore, the omega-limit set $\omega(f)$ is nonempty, compact, and invariant under the translational flow. We list here five examples of functions $f$ for which $H^{+}(f)$ is compact:

(1) $f \in W^{1, \infty}\left((0, \infty), L^{2}(\Omega)\right)$, and there is a compact set $\mathscr{K} \subseteq L^{2}(\Omega)$ such that $f(t) \in \mathscr{K}$ for all $t \geq 0$.

(2) $f \in W^{1, \infty}\left((0, \infty), H^{1}(\Omega)\right)$.

(3) $f(t)$ is continuous and Bohr almost-periodic, or periodic, in $t$.

(4) $f=g+h$, where $g$ and $h$ satisfy (2.51), $\|h(t)\|_{L^{2}(\Omega)} \rightarrow 0$ as $t \rightarrow \infty$, and $g(t)$ is Bohr almost-periodic, or periodic, in $t$.

(5) $f \in L^{2}(\Omega)$ is independent of $t$.

The evaluation mapping $\operatorname{Ev}: W(\Omega) \rightarrow L^{2}(\Omega)$ given by $\operatorname{Ev}(f)=f(0)$ is a continuous mapping of $W(\Omega)$ in $L^{2}(\Omega)$. Therefore if $H^{+}(f)$ is a compact 
set in $W(\Omega)$, then

$$
\operatorname{Ev}\left(H^{+}(f)\right)=\left\{g(0): g \in H^{+}(f)\right\}
$$

is a compact set in $L^{2}(\Omega)$, and one has $g(t) \in \operatorname{Ev}\left(H^{+}(f)\right)$ for all $t \geq 0$ and $g \in H^{+}(f)$.

Let $H$ be a Banach space with a norm $\|\cdot\|_{H}$, and let $\mathscr{F}$ be any positively invariant subset of $W(\Omega)$. Let $\mathscr{O}$ be an open set in $H \times \mathscr{F} \times[0, \infty)$ with

$$
\{(u, f, 0):(u, f) \in H \times \mathscr{F}\} \subset \mathscr{O},
$$

and let $\pi: \mathscr{O} \rightarrow H \times \mathscr{F}$ be a mapping of the form

$$
\pi(u, f ; \tau)=\left(S(f, \tau) u, f_{\tau}\right), \quad(u, f, \tau) \in \mathscr{O} .
$$

For each $(u, f) \in H \times \mathscr{F}$, let $I_{(u, f)}=[0, \tau)$, where $\tau=\tau(u, f)$, denote the maximal time interval for which $(u, f, t) \in \mathscr{O}$ for $0 \leq t<\tau$. We say that $\pi$ is a skew-product semiflow on $H \times \mathscr{F}$ if the following properties are satisfied:

(1) $S(f, 0) u=u$, for all $(u, f) \in H \times \mathscr{F}$.

(2) Whenever $t \in I_{(u, f)}$ and $s \in I_{\left(S(f, t) u, f_{t}\right)}$, then $(t+s) \in I_{(u, f)}$ and one has

$$
S\left(f_{t}, s\right) S(f, t) u=S(f, t+s) u .
$$

(3) The mapping $(u, f, t) \rightarrow \pi(u, f ; t)$ is continuous in $(u, f) \in H \times \mathscr{F}$ for $t$ fixed and continuous in $t$ for $(u, f)$ fixed.

(4) If $(u, f) \in H \times \mathscr{F}$ and $\tau(u, f)<\infty$, then one has

$$
\limsup _{t \rightarrow \tau^{-}}\|S(f, t) u\|_{H}=\infty \text {. }
$$

It is a consequence of (2) that one has

$$
\pi(u, f ; t+s)=\pi(\pi(u, f ; t) ; s) .
$$

Let $\mathscr{K}$ be a subset of $H \times \mathscr{F}$ and assume that $t$ satisfies $0 \leq t<\tau(u, f)$ for all $(u, f) \in \mathscr{K}$. For this $t$ we define $\pi(\mathscr{K} ; t)$ to be the collection of all $\pi(u, f ; t)$ with $(u, f) \in \mathscr{K}$. A subset $\mathscr{K}$ in $H \times \mathscr{F}$ is said to be invariant for $\pi$ if one has $\tau(u, f)=\infty$ for all $(u, f) \in \mathscr{K}$ and $\pi(\mathscr{K} ; t)=\mathscr{K}$ for all $t \geq 0$. If $\mathscr{K}$ is any subset of $H \times \mathscr{F}$ with $\tau(u, f)=\infty$ for all $(u, f) \in \mathscr{K}$, we define the omega-limit set of $\mathscr{K}$ as $\omega(\mathscr{K})$ where

$$
\omega(\mathscr{K})=\bigcap_{\tau \geq 0} \text { Closure }_{H \times \mathscr{F}}\left(\bigcup_{t \geq \tau} \pi(\mathscr{K} ; t)\right) .
$$

In the case of the dilated Navier-Stokes evolutionary equation on $Q_{3}$ with periodic boundary conditions, for $U_{0} \in V_{\varepsilon}^{1}$ and $F \in W\left(Q_{3}\right)$, we set

$$
S(F, t) U_{0}=U(t), \quad 0 \leq t<\tau\left(U_{0}, F\right),
$$

where $U(t)$ is the maximally defined strong solution on $\left[0, \tau\left(U_{0}, F\right)\right)$ that satisfies $U(0)=U_{0}$. One can show that

$$
\mathscr{O} \stackrel{\text { def }}{=}\left\{(U, F, t) \in V_{\varepsilon}^{1} \times W\left(Q_{3}\right) \times[0, \infty): 0 \leq t<\tau(U, F)\right\}
$$


is an open set, and the mapping $\pi$ defined by (2.52) and (2.53) generates a skew-product semiflow on $V_{\varepsilon}^{1} \times W\left(Q_{3}\right)$.

The same construction generates a skew-product semiflow for the NavierStokes equations on any reasonable bounded domain $\Omega$ in $\mathbb{R}^{n}$, for $n=2,3$, and under other homogeneous boundary conditions; see Constantin and Foiaş (1988). In this case, the semiflow is on the space $V^{1} \times W(\Omega)$, where $V^{1}=$ $D\left(A^{1 / 2}\right)$. For the 2DNS, one has $\tau(u, f)=\infty$, for all $(u, f) \in V^{1} \times W(\Omega)$, i.e., $\pi$ is a global semiflow in this case. Furthermore, by using the Leray solutions of the 2DNS instead of the strong solutions, this global semiflow extends to a global semiflow on $H \times H(\Omega)$, where $H=\mathbb{P}_{2} L^{2}(\Omega)$; see Constantin and Foiaş (1988).

For the Navier-Stokes equations we will be studying the semiflow generated by (2.53) on $V_{\varepsilon}^{1} \times \mathscr{F}$, where $\mathscr{F}$ is a compact invariant set in $W\left(Q_{3}\right)$. For example, with equation (2.5) one might assume either $\mathscr{F}=H^{\dagger}(f)$ to be compact, or $\mathscr{F}=H^{+}\left(\mathbb{P}_{\varepsilon} f\right)$ to be compact. Either assumption leads to a reasonable dynamical theory for (2.5). The stronger condition that $H^{+}(f)$ be compact is important primarily in the study of the original system $(2.4)$ where $\left(I-\mathbb{P}_{\varepsilon}\right) f$ is used. Similarly for the reduced 3D Navier-Stokes evolutionary system (2.24), one gets a good dynamical theory by assuming any one of the following three sets to be compact: $H^{+}(f), H^{+}\left(\mathbb{P}_{\varepsilon} f\right)$, or $H^{+}\left(M \mathbb{P}_{\varepsilon} f\right)$.

If the forcing term $f$ is time-independent and in $L^{2}(\Omega)$, then the hull $H(f)$ consists of a single point $\{f\}$, and the Navier-Stokes equations generate (local) semiflows on appropriate Hilbert spaces. For the 3DNS equations the strong solutions $S(t) u_{0}$ generate a semiflow on the Hilbert space $\mathbb{P}_{3}\left(H^{1}(\Omega)\right)$. The weak solutions of the 2DNS generate a semiflow on $\mathbb{P}_{2}\left(L^{2}(\Omega)\right)$.

2.12. Local and global attractors. We will continue to use the notation introduced in $\S 2.11$. Let $\pi$ be the skew-product semiflow on $H \times \mathscr{F}$ given by (2.52), where $\mathscr{F}$ is a compact, positively invariant subset of $W\left(Q_{3}\right)$. A subset $\mathfrak{A}$ in $H \times \mathscr{F}$ is said to be a (local) attractor for $\pi$ if $\mathfrak{A}$ is a compact, invariant set for $\pi$, and $\mathfrak{A}=\omega(U)$ is the omega-limit set of some bounded neighborhood $U$ of $\mathfrak{A}$ in $H \times \mathscr{F}$. The basin of attraction $B(\mathfrak{A})$ defined to be the collection of all $(u, f) \in H \times \mathscr{F}$ with the property that

$$
\operatorname{dist}_{H \times \mathscr{F}}(\pi(u, f ; t), \mathfrak{A}) \rightarrow 0 \text { as } t \rightarrow \infty .
$$

If it happens that $\mathfrak{A}$ is an attractor with $B(\mathfrak{A})=H \times \mathscr{F}$, then $\mathfrak{A}$ is said to be a global attractor for $\pi$. Note that any attractor $\mathfrak{A}$ attracts the bounded set $U$, as well as every compact set $K$ in the basin $B(\mathfrak{A})$. Recall that $\mathfrak{A}$ attracts a set $B \subset H \times \mathscr{F}$ provided that for every $\delta>0$ there is a time $T=T(B, \delta) \geq 0$ such that

$$
\pi(u, f, t) \in N_{(H \times \mathscr{F}, \delta)}(\mathfrak{A}), \quad \text { for all }(u, f) \in B \text { and all } t \geq T,
$$

where $N_{(H \times \mathscr{F}, \delta)}(\mathfrak{A})$ denotes the $\delta$-neighborhood of $\mathfrak{A}$ in $H \times \mathscr{F}$. Recall that the skew-product semiflow $\pi$ is said to be compact, if for every $\tau>0$, the mapping

$$
\pi_{\tau}: \mathscr{O} \rightarrow H \times \mathscr{F}
$$


given by $\pi_{\tau}(u, f)=\pi(u, f, \tau)$, maps bounded sets into compact sets. For a compact semiflow, the attractor $\mathfrak{A}$ attracts all bounded sets in the basin $B(\mathfrak{A})$, see Hale (1988) and Sell and You (1993).

We note that the flow generated by the strong solutions of the 2DNS is compact and always has a global attractor, provided the positive hull $H^{+}(f)$ of the forcing function $f$ is compact. As we shall see, the reduced 3DNS also has a global attractor, when $H^{+}(f)$ is compact. The theorems, which we describe in the next section, effectively state that when $\varepsilon$ is small the full 3DNS has a local attractor $\mathfrak{A}_{\varepsilon}$ and that $\mathfrak{A}_{\varepsilon}$ has a large basin of attraction.

2.13. Statement of theorems about attractors. In this section we assume that $f \in W\left(Q_{3}\right)$ is chosen so that $H^{+}(f)$ is compact; see $\S 2.11$. This includes the case where $f \in \mathrm{L}^{2}\left(Q_{3}\right)$ is time-independent. We assume that Hypothesis $\mathrm{H}(\mathrm{a}$, b) is satisfied, where $a$ and $b$ are sufficiently large. Let $\mathscr{B}_{\varepsilon}^{0}, \mathscr{B}_{\varepsilon}{ }^{1}$, and $\mathscr{B}_{\varepsilon}{ }^{2}$ be given by (2.38), (2.39), and (2.40). It is an immediate consequence of Theorems 1 and 2 that for $u_{0} \in \mathscr{B}_{\varepsilon}^{0} \cup \mathscr{B}_{\varepsilon}{ }^{1}$ the solution $S_{\varepsilon}(f, t) u_{0}=S_{\varepsilon}\left(\mathbb{P}_{\varepsilon} f, t\right) u_{0}$ lies in a bounded set in $V_{\varepsilon}^{2}$ for $t \geq \widehat{T}_{1}$. Therefore, $S_{\varepsilon}(f, t) u_{0}$ lies in a compact set in $V_{\varepsilon}^{1}$ for $t \geq \widehat{T}_{1}$. As a matter of fact, we are able to prove the following compactness result:

Theorem 3. Let $\eta_{i}, i=1,2,3,4, r$, and $p$ satisfy Hypothesis $H(a, b)$, where $a$ and $b$ are sufficiently large. Assume that $f \in W\left(Q_{3}\right)$ is chosen so that $\mathbb{P}_{\varepsilon} f \in$ $W^{1, \infty}\left((0, \infty), H_{\varepsilon}\right), H^{+}(f)$ is compact, and (2.29) is satisfied. Let $S_{\varepsilon}(f, t) u_{0}$ denote the strong solution of (2.5) with initial data $u_{0} \in V_{\varepsilon}^{1}$. Then for any $\tau>0$ there is a compact subset $\mathscr{K}(\tau)$ of $V_{\varepsilon}^{2}$ such that

$$
S_{\varepsilon}(f, t)\left(\mathscr{B}_{\varepsilon}^{0} \cup \mathscr{B}_{\varepsilon}^{1}\right) \subset \mathscr{K}(\tau), \quad t \geq \tau .
$$

The proof of Theorem 3 is given in $\S 4$. If we do not assume $H^{+}(f)$ to be compact in Theorem 3 , then we can only prove, for $t>0, S_{\varepsilon}(t)\left(\mathscr{B}_{\varepsilon}^{0} \cup \mathscr{B}_{\varepsilon}{ }^{1}\right)$ belongs to a compact set $\widehat{\mathscr{K}}(t)$ which may depend on $t$.

The following theorems are proved in $\S 6$. Let $u(t)=S_{\varepsilon}\left(\mathbb{P}_{\varepsilon} f, t\right) u_{0}$ denote the strong solution of the equation (2.5) with initial data $u_{0}$ in $V_{\varepsilon}^{1}$, and let $\pi_{\varepsilon}\left(u_{0}, \mathbb{P}_{\varepsilon} f ; \tau\right)=\left(S_{\varepsilon}\left(\mathbb{P}_{\varepsilon} f, \tau\right) u_{0},\left(\mathbb{P}_{\varepsilon} f\right)_{\tau}\right)$ denote the skew-product semiflow generated by the strong solutions of the dilated 3D Navier-Stokes evolutionary equation $(2.5)$.

Theorem 4. Let $\eta_{i}, i=1,2,3,4, r$, and $p$ satisfy Hypothesis $H(a, b)$, where $a$ and $b$ are sufficiently large. Assume that $f \in W\left(Q_{3}\right)$ is chosen so that $\mathbb{P}_{\varepsilon} f \in W^{1, \infty}\left((0, \infty), H_{\varepsilon}\right), H^{+}(f)$ is compact, and one has

$$
\left\|M \mathbb{P}_{\varepsilon} f\right\|_{\infty}^{2} \leq \eta_{2}^{-2}, \quad\left\|(I-M) \mathbb{P}_{\varepsilon} f\right\|_{\infty}^{2} \leq \varepsilon^{r} \eta_{4}^{-2} .
$$

Let $\varepsilon_{0}>0$ be given by Theorem 1. Then, for $0<\varepsilon \leq \varepsilon_{0}$, the skew-product flow $\pi_{\varepsilon}$ generated by the strong solutions of the dilated 3D Navier-Stokes evolutionary equation (2.5) has a unique, maximal, compact (local) attractor $\mathfrak{A}_{\varepsilon}$ included in 
$\mathscr{B}_{\varepsilon}^{2} \times \omega\left(\mathbb{P}_{\varepsilon} f\right)$, which attracts $\mathscr{B}_{\varepsilon}^{2} \times H^{+}\left(\mathbb{P}_{\varepsilon} f\right)$ in the space $V_{\varepsilon}^{1} \times \mathbb{P}_{\varepsilon} W\left(Q_{3}\right)$, where $\mathscr{B}_{\varepsilon}^{2}$ is given by (2.40). Furthermore, one has

$$
\mathfrak{A}_{\varepsilon} \subset\left\{u=v+w:\left\|A_{\varepsilon}^{1 / 2} v\right\|^{2} \leq \Gamma\left(\eta_{2}^{-2}\right),\left\|A_{\varepsilon}^{1 / 2} w\right\|^{2} \leq k_{2}^{2} \varepsilon^{2+r} \eta_{4}^{-2}\right\} \times \omega\left(\mathbb{P}_{\varepsilon} f\right) .
$$

Moreover, $\mathfrak{A}_{\varepsilon}$ is bounded and compact in $V_{\varepsilon}^{2} \times \omega\left(\mathbb{P}_{\varepsilon} f\right)$ and attracts the bounded set $\left(\mathscr{B}_{\varepsilon}^{2} \cap V_{\varepsilon}^{2}\right) \times H^{+}\left(\mathbb{P}_{\varepsilon} f\right)$ in the space $V_{\varepsilon}^{2} \times \mathbb{P}_{\varepsilon} W\left(Q_{3}\right)$.

In the next result we show that, under an added condition on $\eta_{i}, i=$ $1,2,3,4$, see (2.55) below, the attractor $\mathfrak{A}_{\varepsilon}$ is the global attractor for the Leray solutions of the dilated 3D Navier-Stokes evolutionary equation (2.5), i.e., the weak solutions that satisfy the energy inequality (3.35); see Foias and Temam (1987). ${ }^{4}$ Note that the example given in Remark 2 prior to the statement of Theorem 1 satisfies (2.55).

Corollary 4.1. Let the hypotheses of Theorem 4 be satisfied. Assume in addition that for every $\lambda>0$ there is an $\varepsilon_{10}=\varepsilon_{10}(\lambda)>0$ such that

$$
\eta_{2}^{-2}+\varepsilon^{2+r} \eta_{4}^{-2} \leq \lambda \min \left(\eta_{1}^{-2}, \varepsilon^{p} \eta_{3}^{-2}\right), \quad 0<\varepsilon \leq \min \left(\varepsilon_{0}, \varepsilon_{10}\right) .
$$

Then for any $\varepsilon$ satisfying $0<\varepsilon \leq \min \left(\varepsilon_{0}, \varepsilon_{10}\right)$ and any $\rho>0$, there is a time $T=T(\rho, \varepsilon)>0$, and, for every weak (Leray) solution $u(t)$ of $(2.5)$ with $\|u(0)\| \leq \rho$, there is a time $t_{0}$ satisfying $0<t_{0} \leq T(\rho, \varepsilon)$ and $u\left(t_{0}\right) \in \mathscr{B}_{\varepsilon}^{0}$. In particular, $u(t)$ is a regular solution of (2.5) for $t \geq t_{0}$, and the attractor $\mathfrak{A}_{\varepsilon}$ given in Theorem 4 is the global attractor for the Leray solutions of (2.5), provided $0<\varepsilon \leq \min \left(\varepsilon_{0}, \varepsilon_{10}\right)$.

Let us now consider the reduced 3D Navier-Stokes evolutionary equation (2.24), and let us denote by $S_{0}(g, t) \bar{v}_{0}$ the strong solution of (2.24) with initial data $\bar{v}_{0}$ in $M V_{\varepsilon}^{1}$, where $g=M \mathbb{P}_{\varepsilon} f$. We denote by $\pi_{0}\left(\bar{v}_{0}, g ; \tau\right)=$ $\left(S_{0}(g, \tau) \bar{v}_{0}, g_{\tau}\right)$ the skew-product semiflow generated by the strong solutions of (2.24). As noted in $\S 2.6$, the terms in (2.24) do not depend on $x_{3}$ and $\varepsilon$. We have the following result:

Corollary 4.2. Assume that $f \in W\left(Q_{3}\right)$ is chosen so that $\mathbb{P}_{\varepsilon} f \in W^{1, \infty}\left((0, \infty) ; H_{\varepsilon}\right)$ and $H^{+}(f)$ is compact. Then $\pi_{0}$ admits a global attractor $\mathfrak{A}_{0}=\mathfrak{A}_{0}(g)$ in $M V_{\varepsilon}^{1} \times H^{+}(g)$. Furthermore, if $\left\|M \mathbb{P}_{\varepsilon} f\right\|_{\infty}^{2} \leq \eta_{2}^{-2}$, where $\eta_{2}$ is given by Hypothesis $H(a, b)$, then

(2.56) $\mathfrak{A}_{0}(g) \subset\left\{u=v+w: v \in M V_{\varepsilon}^{1},\left\|A_{\varepsilon}^{1 / 2} v\right\|^{2} \leq \Gamma\left(\eta_{2}^{-2}\right), w=0\right\} \times \omega(g)$.

If, in addition, one has

$$
(I-M) \mathbb{P}_{\varepsilon} f=0,
$$

then the attractors $\mathfrak{A}_{\varepsilon}$ and $\mathfrak{A}_{0}(g)$ coincide for $0<\varepsilon \leq \varepsilon_{0}$, where $\varepsilon_{0}$ is given by Theorem 1.

If $(I-M) \mathbb{P}_{\varepsilon} f \neq 0$, a comparison of the two attractors $\mathfrak{A}_{\varepsilon}$ and $\mathfrak{A}_{0}(g)$ is more difficult. Nevertheless, we are able to derive some results establishing the upper semicontinuity of $\mathfrak{A}_{\varepsilon}$ at $\varepsilon=0$.

\footnotetext{
${ }^{4}$ Recall that the 3DNS can have weak solutions that do not satisfy the energy inequality (3.35); see Temam (1983).
} 
Let us consider a sequence of positive numbers $\varepsilon_{n} \rightarrow 0$ when $n \rightarrow \infty$. We introduce a sequence of functions $f_{n}$ in $W\left(Q_{3}\right) \cap W^{1, \infty}\left((0, \infty), L^{2}\left(Q_{3}\right)\right)$ such that $f_{n} \rightarrow f_{0}$ in $W\left(Q_{3}\right)$, where $f_{0} \in M W\left(Q_{3}\right) \cap W^{1, \infty}\left((0, \infty) ; M L^{2}\left(Q_{3}\right)\right)$. We set $g_{n}=\mathbb{P}_{\varepsilon_{n}} f_{n}$ and $g_{0}=M \mathbb{P}_{\varepsilon_{n}} f_{0}$. According to the comments made in $\S 2.6, \mathbb{P}_{\varepsilon_{n}} f_{0}(t)$ belongs to $M H_{\varepsilon_{n}}$, for every $t$, and consequently

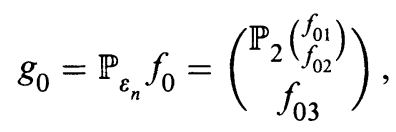

where $f_{0}=\left(f_{01}, f_{02}, f_{03}\right)$. It follows from the above convergence hypothesis and from (2.57) that

$$
\lim _{n \rightarrow \infty}\left\|g_{n}-g_{0}\right\|_{\infty}=0
$$

We consider next the reduced 3D Navier-Stokes evolutionary equation

$$
\bar{v}^{\prime}+\nu A_{0} \bar{v}+B_{0}(\bar{v}, \bar{v})=g_{0}
$$

with initial data $\bar{v}(0)=\bar{v}_{0}$ in $V_{0}^{1}$, and we let $S_{0}\left(g_{0}, t\right) \bar{v}_{0}$ denote the strong solutions of (2.58) with initial data $\bar{v}_{0}$ in $V_{0}^{1}$. As a consequence of Corollary 4.2, the skew-product semiflow $\pi_{0}\left(\bar{v}_{0}, g_{0} ; \tau\right)=\left(S_{0}\left(g_{0}, \tau\right) \bar{v}_{0}, g_{0 \tau}\right)$ admits a global compact attractor $\mathfrak{A}_{0}=\mathfrak{A}_{0}\left(g_{0}\right)$ in $V_{0}^{1} \times \omega\left(g_{0}\right)$, which is also the global compact attractor in $V_{0}^{2} \times \omega\left(g_{0}\right)$.

Let $E$ be a subset of $V_{\varepsilon_{n}}^{1} \times W\left(Q_{3}\right)$. For any $\delta>0$, we denote by $\mathscr{N}_{V_{\varepsilon_{n}}^{1} \times W\left(Q_{3}\right)}(E, \delta)$ the $\delta$-neighborhood of $E$ in $V_{\varepsilon_{n}}^{1} \times W\left(Q_{3}\right)$. We will prove the following result:

Theorem 5. Let $\eta_{i}, i=1,2,3,4, r$, and $p$ satisfy Hypothesis $H(a, b)$, where $a$ and $b$ are sufficiently large, and assume that

$$
\varepsilon^{4+2 r} \eta_{4}^{-4}(\varepsilon) \rightarrow 0 \text { as } \varepsilon \rightarrow 0^{+} .
$$

Let $\varepsilon_{n}$ be a sequence of positive numbers with $\varepsilon_{n} \rightarrow 0$ as $n \rightarrow \infty$. Let $\mathscr{F}$ be any positively invariant compact subset of $W\left(Q_{3}\right) \cap W^{1, \infty}\left((0, \infty), L^{2}\left(Q_{3}\right)\right)$, and let $f_{n}$ be a sequence of functions in $\mathscr{F}$ that satisfies

$$
\lim _{n \rightarrow \infty}\left\|f_{n}-f_{0}\right\|_{\infty}=0
$$

where $f_{0} \in M \mathscr{F}$. Assume further that

$$
\left\|M \mathbb{P}_{\varepsilon_{n}} f_{n}\right\|_{\infty}^{2}<\eta_{2}^{-2}, \quad\left\|(I-M) \mathbb{P}_{\varepsilon_{n}} f_{n}\right\|_{\infty}^{2} \leq \varepsilon_{n}^{r} \eta_{4}^{-2} .
$$

Then the attractors $\mathfrak{A}_{\varepsilon_{n}}$, given in Theorem 4 , are upper semicontinuous in $V_{\varepsilon_{n}}^{1} \times \mathscr{F}$ at $\varepsilon=0$, i.e., for any $\delta>0$, there is an $n_{0}>0$ such that

$$
\mathfrak{A}_{\varepsilon_{n}} \subset \mathscr{N}_{V_{\varepsilon_{n}}^{1} \times W\left(Q_{3}\right)}\left(\mathfrak{A}_{0}\left(g_{0}\right), \delta\right)
$$

for $n \geq n_{0}$, where $g_{0}=M \mathbb{P}_{\varepsilon_{n}} f_{0}=\mathbb{P}_{\varepsilon_{n}} f_{0}$.

Theorem 5 has some interesting extensions. The following result, which we formulate in the case where the forcing terms $f_{n}$ are independent of time $t$, 
allows for the possibility that $f_{n}$ can be chosen so that

$$
\left\|(I-M) \mathbb{P}_{\varepsilon_{n}} f_{n}\right\|^{2} \rightarrow \infty \quad \text { as } n \rightarrow \infty .
$$

If $f$ is independent of $t$, the mapping $S_{\varepsilon}(t) \stackrel{\text { def }}{=} S_{\varepsilon}\left(\mathbb{P}_{\varepsilon} f, t\right)$ is a (local) $C^{0}$ semigroup on $V_{\varepsilon}^{1}$. We then deduce from Theorem 4 that $S_{\varepsilon}(t)$ admits a unique maximal compact (local) attractor $\widetilde{\mathfrak{A}}_{\varepsilon}$ included in $\mathscr{B}_{\varepsilon}^{2}$ which attracts $\mathscr{B}_{\varepsilon}^{2}$ in the space $V_{\varepsilon}^{1}$. Actually, we have

$$
\mathfrak{A}_{\varepsilon}=\widetilde{\mathfrak{A}}_{\varepsilon} \times\left\{\mathbb{P}_{\varepsilon} f\right\} .
$$

Likewise, $S_{0}(t) \stackrel{\text { def }}{=} S_{0}(g, t)$ is a $C^{0}$-semigroup on $V_{0}^{1}=M V_{\varepsilon}^{1}$, where $g=$ $M \mathbb{P}_{\varepsilon} f$. We deduce from Corollary 4.2 that $S_{0}(t)$ admits a global, compact attractor $\widetilde{\mathfrak{A}}_{0}=\widetilde{\mathfrak{A}}_{0}(g)$ in $V_{0}^{1}$; and we have

$$
\mathfrak{A}_{0}=\tilde{\mathfrak{A}}_{0} \times\{g\} .
$$

The following result is proved in $\S 6$.

Corollary 5.1. Let $\eta_{i}, i=1,2,3,4, r$, and $p$ satisfy Hypothesis $H(a, b)$, where $a$ and $b$ are sufficiently large, and assume that (2.59) holds. Let $\varepsilon_{n}$ be a sequence of positive numbers with $\varepsilon_{n} \rightarrow 0$ as $n \rightarrow \infty$. Let $f_{n}$ be a sequence in $L^{2}\left(Q_{3}\right)$ that satisfies

$$
\lim _{n \rightarrow \infty}\left\|M \mathbb{P}_{\varepsilon_{n}} f_{n}-g_{0}\right\|=0
$$

for some $g_{0} \in H_{0}$. Assume further that

$$
\left\|M \mathbb{P}_{\varepsilon_{n}} f_{n}\right\|^{2} \leq \eta_{2}^{-2}, \quad\left\|(I-M) \mathbb{P}_{\varepsilon_{n}} f_{n}\right\|^{2} \leq \varepsilon_{n}^{r} \eta_{4}^{-2} .
$$

Then the attractors $\widetilde{\mathfrak{A}}_{\varepsilon_{n}}$ of (2.5) with forcing term $\mathbb{P}_{\varepsilon_{n}} f_{n}$ are upper semicontinuous at $\varepsilon=0$ in $V_{\varepsilon_{n}}^{1}$, i.e.,

$$
\sup _{u_{n} \in \widetilde{\mathfrak{A}}_{\varepsilon_{n}}} \inf _{v \in \widetilde{\mathfrak{A}}_{0}}\left\|A_{\varepsilon_{n}}^{1 / 2}\left(u_{n}-v\right)\right\| \rightarrow 0 \text { as } \varepsilon=\varepsilon_{n} \rightarrow 0,
$$

where $\widetilde{\mathfrak{A}}_{0}=\widetilde{\mathfrak{A}}_{0}\left(g_{0}\right)$ is the global attractor of $(2.58)$.

Using the fact that $\mathfrak{A}_{0}=\mathfrak{A}_{0}\left(g_{0}\right)$ is also the global compact attractor of the skew-product semiflow $\pi_{0}\left(\cdot, g_{0} ; \tau\right)$ in $V_{0}^{2} \times H^{+}\left(g_{0}\right)$, one also obtains the result:

Corollary 5.2. Assume that the hypotheses of Theorem 5 hold and that

$$
\lim _{n \rightarrow \infty}\left\|f_{n}^{\prime}-f_{0}^{\prime}\right\|_{\infty}=0 \text {. }
$$

Then the attractors $\mathfrak{A}_{\varepsilon_{n}}$ are also upper semicontinuous at $\varepsilon=0$ in $V_{\varepsilon}^{2} \times W\left(Q_{3}\right)$, i.e., for any $\delta>0$, there exists an integer $n_{1}>0$ such that, for $n \geq n_{1}$,

$$
\mathfrak{A}_{\varepsilon_{n}} \subset \mathscr{N}_{V_{\varepsilon_{n}}^{2} \times W\left(Q_{3}\right)}\left(\mathfrak{A}_{0}\left(g_{0}\right), \delta\right) .
$$


Remark. We now give an example where the condition (2.60) is satisfied. Let $F=F(t, y)$ be given, where $F \in C^{0}\left(\mathbb{R}, W^{1, \infty}\left(Q_{3}\right)\right) \cap L^{\infty}\left(\mathbb{R}, W^{1, \infty}\left(Q_{3}\right)\right)$. As in $\S 2.1$, we introduce the mapping $f_{\varepsilon}$ by setting $F=J_{\varepsilon} f_{\varepsilon}$

$$
f_{\varepsilon}\left(t, x_{1}, x_{2}, x_{3}\right)=F\left(t, x_{1}, x_{2}, \varepsilon x_{3}\right), \quad\left(x_{1}, x_{2}, x_{3}\right) \in Q_{3} .
$$

Next set $f_{0}\left(t, x_{1}, x_{2}, x_{3}\right)=F\left(t, x_{1}, x_{2}, 0\right)$. By applying the integral Taylor formula, we obtain

$$
\begin{aligned}
f_{\varepsilon}(t, x)-f_{0}(t, x) & =F\left(t, x_{1}, x_{2}, \varepsilon x_{3}\right)-F\left(t, x_{1}, x_{2}, 0\right) \\
& =\varepsilon \int_{0}^{1} \frac{\partial F}{\partial x_{3}}\left(t, x_{1}, x_{2}, s x_{3}\right) x_{3} d s
\end{aligned}
$$

and therefore

$$
\left\|f_{\varepsilon}-f_{0}\right\|_{C^{0}\left([0, \infty) ; L^{\infty}\left(Q_{3}\right)\right)} \leq \varepsilon\left\|D_{3} F\right\|_{C^{0}\left([0, \infty) ; L^{\infty}\left(Q_{3}\right)\right)} .
$$

In particular, we have

$$
\left\|f_{\varepsilon}-f_{0}\right\|_{\infty} \leq C \varepsilon
$$

If, in addition, $F$ belongs to $C^{1}\left(\mathbb{R}, W^{1, \infty}\left(Q_{3}\right)\right) \cap L^{\infty}\left(\mathbb{R}, W^{1, \infty}\left(Q_{3}\right)\right)$, then the condition (2.62) is also satisfied; more precisely, we have

$$
\left\|f_{\varepsilon}^{\prime}-f_{0}^{\prime}\right\|_{\infty} \leq \widetilde{C} \varepsilon
$$

\section{3. $H^{1}$-Regularity: THEOREM 1}

In this section we prove Theorem 1 , the $H^{1}$-Regularity Theorem, which gives the global regularity of the Navier-Stokes equations on $\Omega_{\varepsilon}$ in the Sobolev space $H^{1}$. We assume that the forcing function $f$ is in $L^{\infty}\left((0, \infty), L^{2}\left(Q_{3}\right)\right)$ and that the initial condition $u_{0}$ satisfies $u_{0} \in D\left(A_{\varepsilon}^{1 / 2}\right)$. Also we assume that one has

$$
\begin{cases}\left\|A_{\varepsilon}^{1 / 2} v_{0}\right\|^{2} \leq \eta_{1}^{-2}, & \left\|M \mathbb{P}_{\varepsilon} f\right\|_{\infty}^{2} \leq \eta_{2}^{-2}, \\ \left\|A_{\varepsilon}^{1 / 2} w_{0}\right\|^{2} \leq \varepsilon^{p} \eta_{3}^{-2}, & \left\|(I-M) \mathbb{P}_{\varepsilon} f\right\|_{\infty}^{2} \leq \varepsilon^{r} \eta_{4}^{-2},\end{cases}
$$

where $\eta_{i}(\varepsilon)$ is bounded and monotone for $0<\varepsilon \leq 1, i=1,2,3,4$. (We are primarily interested in the case where $\eta_{i}(\varepsilon) \rightarrow 0$ as $\varepsilon \rightarrow 0, i=1,2,3,4$.) Throughout this section we let $D_{1}, D_{2}, \ldots$ denote positive functions of the viscosity $\nu$ and $\lambda_{1}$, the first eigenvalue of $A_{\varepsilon}$. These functions will not depend on $\varepsilon$ for $0<\varepsilon \leq 1$.

The proof of the $H^{1}$-Regularity Theorem is done in two steps. In the first lemma, which is the Short Time Argument, we show that the $w(t)$-term becomes small very rapidly. The second lemma is referred to as the Long Time Argument. This is the induction step needed for the proof of the $H^{1}$-Regularity Theorem. (See $\S \S 3.2$ and 3.3.)

We will use the following auxiliary estimates concerning the trilinear form $b_{\varepsilon}$ : If $v^{1}, v^{2}, v^{3} \in R(M)$, then these functions depend only on $x_{1}$ and $x_{2}$, and one has

$$
\left|b_{\varepsilon}\left(v^{1}, v^{2}, v^{3}\right)\right| \leq C_{1}\left\|v^{1}\right\|^{1 / 2}\left\|A_{\varepsilon}^{1 / 2} v^{1}\right\|^{1 / 2}\left\|A_{\varepsilon}^{1 / 2} v^{2}\right\|^{1 / 2}\left\|A_{\varepsilon} v^{2}\right\|^{1 / 2}\left\|v^{3}\right\| .
$$


The proof of (3.2) is accomplished by using 2D Sobolev embeddings; see Temam (1983) or Constantin and Foiaş (1988) for details. If one has $v \in R(M)$ and $M w^{1}=M w^{2}=M w=0$, then the following inequalities hold:

$$
\left\{\begin{array}{l}
\left|b_{\varepsilon}\left(w^{1}, w^{2}, u\right)\right| \leq C_{2} \varepsilon^{1 / 2}\left\|A_{\varepsilon}^{1 / 2} w^{1}\right\|\left\|A_{\varepsilon}^{1 / 2} w^{2}\right\|^{1 / 2}\left\|A_{\varepsilon} w^{2}\right\|^{1 / 2}\|u\| \\
\left|b_{\varepsilon}\left(w, u^{2}, u^{3}\right)\right| \leq C_{3} \varepsilon^{5 / 32}\left\|A_{\varepsilon}^{1 / 2} w\right\|^{15 / 32}\left\|A_{\varepsilon} w\right\|^{17 / 32}\left\|A_{\varepsilon}^{1 / 2} u^{2}\right\|\left\|u^{3}\right\| \\
\left|b_{\varepsilon}(v, w, u)\right| \leq C_{4} \varepsilon^{1 / 4}\left\|A_{\varepsilon}^{1 / 2} v\right\|\left\|A_{\varepsilon}^{1 / 2} w\right\|^{1 / 2}\left\|A_{\varepsilon} w\right\|^{1 / 2}\|u\| .
\end{array}\right.
$$

The proof of (3.3) is given in the appendix, $\S 8$. It is important to note that the constants $C_{1}, C_{2}, C_{3}$, and $C_{4}$ above do not depend on $\varepsilon$, for $0<\varepsilon \leq 1$.

We shall also use the Young inequality

$$
a b \leq \frac{c^{p} a^{p}}{p}+\frac{b^{q}}{q c^{q}}=\delta a^{p}+c_{\delta} b^{q},
$$

where $a, b, c, \delta$, and $c_{\delta}$ are positive, $1 \leq p, q, p^{-1}+q^{-1}=1$, as well as

$$
(a+b)^{3} \leq 4\left(a^{3}+b^{3}\right), \quad a, b \geq 0 .
$$

The proof of the following result can easily be derived from the theory presented in Constantin and Foias (1988), Temam (1977, Chapter III, Lemma 1.2 and Theorem 3.11), Temam (1983, §3, Theorem 3.2), as well as the other references cited above.

Lemma 3.0. Let $u_{0} \in D\left(A_{\varepsilon}^{1 / 2}\right)$ and $f \in L^{\infty}\left(0, T ; H_{\varepsilon}\right)$. Then there exists $a$ time $T_{*}, 0<T_{*} \leq \infty$, such that there exists a unique solution $u$ of $(2.5)$ on $\left(0, T_{*}\right)$. Moreover, $u$ satisfies: $u \in C^{0}\left(\left[0, T_{*}\right] ; V_{\varepsilon}^{1}\right) \cap L^{2}\left(0, T_{*} ; V_{\varepsilon}^{2}\right)$, and $u_{t} \in$ $L^{2}\left(0, T_{*} ; H_{\varepsilon}\right)$. Assume furthermore that $R_{0}^{2} \geq\left\|A_{\varepsilon}^{1 / 2} u_{0}\right\|^{2}$ and $N>1$. Then there exists a positive time $T^{N}, 0<T^{N} \leq T_{*}$, such that $\left\|A_{\varepsilon}^{1 / 2} u(t)\right\|^{2} \leq N R_{0}^{2}$ for $0 \leq t<T^{N}$.

3.1. The short time argument. We shall say that Hypothesis $\mathbf{H 1}$ is satisfied if one has:

(1) $p \geq-1, r>-2$.

(2) $\varepsilon^{1 / 4} \eta_{i}^{-1} \rightarrow 0$ as $\varepsilon \rightarrow 0, i=1,2$.

(3) $\varepsilon^{1 / 8} \eta_{i}^{-1} \rightarrow 0$ as $\varepsilon \rightarrow 0, i=3,4$.

(4) $\varepsilon^{1 / 4} Q(\varepsilon)$ is bounded for $0<\varepsilon \leq 1$, where

$$
Q(\varepsilon)=\left|\log \left(2 C_{5}^{2} \nu^{-2} \varepsilon^{2+r-p} \eta_{4}^{-2} \eta_{3}^{2}\right)\right|
$$

Here $\eta_{i}(\varepsilon)$ denote bounded monotone functions defined for $0<\varepsilon \leq 1$, $i=$ $1,2,3,4$, and $r$ and $p$ are negative constants.

We now prove the following result.

Lemma 3.1. Assume that Hypothesis $H 1$ is satisfied and that (3.1) is valid. Then there are positive constants $k_{1}$ and $k_{2}$ and an $\varepsilon_{1}>0$ such that for all $0<\varepsilon \leq \varepsilon_{1}$ there exists a time $T_{1}=T_{1}(\varepsilon)>0$ such that $u(t) \in D\left(A_{\varepsilon}^{1 / 2}\right)$ for $0 \leq t \leq T_{1}$ and

$$
\left\{\begin{array}{l}
\left\|A_{\varepsilon}^{1 / 2} v\left(T_{1}\right)\right\|^{2} \leq 4 \eta_{1}^{-2}+k_{1}^{2} \eta_{3}^{-4} \\
\left\|A_{\varepsilon}^{1 / 2} w\left(T_{1}\right)\right\|^{2} \leq k_{2}^{2} \varepsilon^{2+r} \eta_{4}^{-2}
\end{array}\right.
$$


Proof. Define $R_{0}^{2}$ by

$$
R_{0}^{2} \stackrel{\text { def }}{=} \eta_{1}^{-2}+\varepsilon^{p} \eta_{3}^{-2}+\eta_{3}^{-4}+\eta_{2}^{-2}+\varepsilon^{r / 3} \eta_{4}^{-2}
$$

Since $R_{0}^{2} \geq\left\|A_{\varepsilon}^{1 / 2} u_{0}\right\|^{2}$, it follows from Lemma 3.0 that for any $N>1$ there is a time $T^{N}>0$ such that

$$
\left\|A_{\varepsilon}^{1 / 2} u(t)\right\|^{2} \leq N R_{0}^{2}, \quad 0 \leq t<T^{N}
$$

Without loss of generality we let $\left[0, T^{N}\right)$ denote the maximal time interval for which (3.9) is valid. If $T^{N}<\infty$, then one must have

$$
\left\|A_{\varepsilon}^{1 / 2} u\left(T^{N}\right)\right\|^{2}=N R_{0}^{2}
$$

For the remainder of the proof of this lemma we restrict our attention to $t \in$ $\left(0, T^{N}\right)$.

The equation satisfied by $w=(I-M) u$ in $(2.23)$ is

$$
\text { 1) } \frac{d w}{d t}+\nu A_{\varepsilon} w=(I-M) \mathbb{P}_{\varepsilon} f-(I-M)\left(B_{\varepsilon}(w, v)+B_{\varepsilon}(v, w)+B_{\varepsilon}(w, w)\right)
$$

since $(I-M) B_{\varepsilon}(v, v)=0$. By taking the scalar product of (3.11) with $A_{\varepsilon} w$, we obtain

$$
\begin{aligned}
\frac{1}{2} \frac{d}{d t}\left\|A_{\varepsilon}^{1 / 2} w\right\|^{2}+\nu\left\|A_{\varepsilon} w\right\|^{2} \leq & \left|\left\langle(I-M) \mathbb{P}_{\varepsilon} f, A_{\varepsilon} w\right\rangle\right|+\left|b_{\varepsilon}\left(w, v, A_{\varepsilon} w\right)\right| \\
& +\left|b_{\varepsilon}\left(v, w, A_{\varepsilon} w\right)\right|+\left|b_{\varepsilon}\left(w, w, A_{\varepsilon} w\right)\right| .
\end{aligned}
$$

By using (3.3) and the Young inequality (3.4) we obtain

$$
\begin{aligned}
\frac{1}{2} \frac{d}{d t}\left\|A_{\varepsilon}^{1 / 2} w\right\|^{2}+\nu\left\|A_{\varepsilon} w\right\|^{2} \leq & \frac{\nu}{2}\left\|A_{\varepsilon} w\right\|^{2}+\frac{1}{2 \nu}\left\|(I-M) \mathbb{P}_{\varepsilon} f\right\|_{\infty}^{2} \\
& +C_{3} \varepsilon^{5 / 32}\left\|A_{\varepsilon}^{1 / 2} v\right\|\left\|A_{\varepsilon}^{1 / 2} w\right\|^{15 / 32}\left\|A_{\varepsilon} w\right\|^{49 / 32} \\
& +C_{4} \varepsilon^{1 / 4}\left\|A_{\varepsilon}^{1 / 2} v\right\|\left\|A_{\varepsilon}^{1 / 2} w\right\|^{1 / 2}\left\|A_{\varepsilon} w\right\|^{3 / 2} \\
& +C_{2} \varepsilon^{1 / 2}\left\|A_{\varepsilon}^{1 / 2} w\right\|^{3 / 2}\left\|A_{\varepsilon} w\right\|^{3 / 2} .
\end{aligned}
$$

Since $M w=0$, one can use (2.22) together with (3.12) to find

$$
\begin{aligned}
\frac{d}{d t}\left\|A_{\varepsilon}^{1 / 2} w\right\|^{2}+\nu\left\|A_{\varepsilon} w\right\|^{2} \leq & \frac{1}{\nu}\left\|(I-M) \mathbb{P}_{\varepsilon} f\right\|_{\infty}^{2}+2 C_{5}^{15 / 32} C_{3} \varepsilon^{5 / 8}\left\|A_{\varepsilon}^{1 / 2} v\right\|\left\|A_{\varepsilon} w\right\|^{2} \\
& +2 C_{5}^{1 / 2} C_{4} \varepsilon^{3 / 4}\left\|A_{\varepsilon}^{1 / 2} v\right\|\left\|A_{\varepsilon} w\right\|^{2} \\
& +2 C_{5}^{1 / 2} C_{2} \varepsilon\left\|A_{\varepsilon}^{1 / 2} w\right\|\left\|A_{\varepsilon} w\right\|^{2} .
\end{aligned}
$$

From the Pythagorean relation (2.21) one obtains

$$
\frac{d}{d t}\left\|A_{\varepsilon}^{1 / 2} w\right\|^{2}+\left(\nu-D_{1} \varepsilon^{5 / 8}\left\|A_{\varepsilon}^{1 / 2} u\right\|\right)\left\|A_{\varepsilon} w\right\|^{2} \leq \frac{1}{\nu}\left\|(I-M) \mathbb{P}_{\varepsilon} f\right\|_{\infty}^{2},
$$

where $D_{1}=2\left(C_{5}^{15 / 32} C_{3}+C_{5}^{1 / 2} C_{4}+C_{5}^{1 / 2} C_{2}\right)$. From Hypothesis $H 1$ we see that for $0 \leq t<T^{N}$ one has

$$
D_{1} \varepsilon^{5 / 8}\left\|A_{\varepsilon}^{1 / 2} u\right\| \leq D_{1} \varepsilon^{5 / 8} N^{1 / 2} R_{0} \leq D_{1} N^{1 / 2} \varepsilon^{5 / 8}\left(\eta_{1}^{-1}+\varepsilon^{p / 2} \eta_{3}^{-1}+\eta_{3}^{-2}+\eta_{2}^{-1}+\varepsilon^{r / 6} \eta_{4}^{-1}\right)
$$


which goes to 0 , as $\varepsilon \rightarrow 0^{+}$. Consequently there is a positive number $\varepsilon_{2}=$ $\varepsilon_{2}(N)$ that satisfies

$$
D_{1} N^{1 / 2} \varepsilon^{5 / 8} R_{0} \leq \frac{\nu}{2}, \quad 0<\varepsilon \leq \varepsilon_{2} .
$$

(Later $N$ will be fixed, and it will depend only on $\nu$ and $\lambda_{1}$.) For $0<\varepsilon \leq \varepsilon_{2}$ and $0 \leq t<T^{N}$ it follows from (3.13), (3.14), and (3.15) that

$$
\frac{d}{d t}\left\|A_{\varepsilon}^{1 / 2} w\right\|^{2}+\frac{\nu}{2}\left\|A_{\varepsilon} w\right\|^{2} \leq \frac{1}{\nu}\left\|(I-M) \mathbb{P}_{\varepsilon} f\right\|_{\infty}^{2}
$$

and from (2.22) that

$$
\frac{d}{d t}\left\|A_{\varepsilon}^{1 / 2} w\right\|^{2}+\frac{\nu C_{5}^{-2} \varepsilon^{-2}}{2}\left\|A_{\varepsilon}^{1 / 2} w\right\|^{2} \leq \frac{1}{\nu}\left\|(I-M) \mathbb{P}_{\varepsilon} f\right\|_{\infty}^{2} .
$$

We then apply the Gronwall inequality to (3.17) to obtain

$$
\left\|A_{\varepsilon}^{1 / 2} w(t)\right\|^{2} \leq \exp \left(\frac{-\nu C_{5}^{-2} \varepsilon^{-2}}{2} t\right)\left\|A_{\varepsilon}^{1 / 2} w_{0}\right\|^{2}+\frac{2 C_{5}^{2} \varepsilon^{2}}{\nu^{2}}\left\|(I-M) \mathbb{P}_{\varepsilon} f\right\|_{\infty}^{2}
$$

for $0 \leq t<T^{N}$ and $0<\varepsilon \leq \varepsilon_{2}$. By integrating (3.16) we also obtain

$$
\int_{0}^{t}\left\|A_{\varepsilon} w(s)\right\|^{2} d s \leq \frac{2 t}{\nu^{2}}\left\|(I-M) \mathbb{P}_{\varepsilon} f\right\|_{\infty}^{2}+\frac{2}{\nu}\left\|A_{\varepsilon}^{1 / 2} w_{0}\right\|^{2}
$$

for $0 \leq t<T^{N}$ and $0<\varepsilon \leq \varepsilon_{2}$.

For the remainder of the proof we shall restrict our attention to $0<\varepsilon \leq \varepsilon_{2}$. We will need an estimate of $\int_{0}^{t}\left\|A_{\varepsilon}^{1 / 2} w\right\|^{3}\left\|A_{\varepsilon} w\right\| d s$. From (3.5) and (3.18) we obtain

$$
\begin{aligned}
\int_{0}^{t}\left\|A_{\varepsilon}^{1 / 2} w\right\|^{6} d s \\
\quad \leq 4 \int_{0}^{t}\left[\exp \left(\frac{-3 \nu C_{5}^{-2} \varepsilon^{-2}}{2} s\right)\left\|A_{\varepsilon}^{1 / 2} w_{0}\right\|^{6}+\frac{8 C_{5}^{6} \varepsilon^{6}}{\nu^{6}}\left\|(I-M) \mathbb{P}_{\varepsilon} f\right\|_{\infty}^{6}\right] d s \\
\quad \leq 4\left(\frac{2 C_{5}^{2} \varepsilon^{2}}{3 \nu}\left\|A_{\varepsilon}^{1 / 2} w_{0}\right\|^{6}+\frac{8 C_{5}^{6} \varepsilon^{6} t}{\nu^{6}}\left\|(I-M) \mathbb{P}_{\varepsilon} f\right\|_{\infty}^{6}\right) .
\end{aligned}
$$

By using the Schwarz inequality with (3.19) and (3.20) we next obtain

$$
\begin{aligned}
\int_{0}^{t}\left\|A_{\varepsilon}^{1 / 2} w\right\|^{3}\left\|A_{\varepsilon} w\right\| d s \leq\left(\int_{0}^{t}\left\|A_{\varepsilon}^{1 / 2} w\right\|^{6} d s\right)^{1 / 2}\left(\int_{0}^{t}\left\|A_{\varepsilon} w\right\|^{2} d s\right)^{1 / 2} \\
\leq 4 C_{5} \nu^{-1} \varepsilon\left(\frac{1}{\sqrt{3}}\left\|A_{\varepsilon}^{1 / 2} w_{0}\right\|^{3}+\frac{2 C_{5}^{2} \varepsilon^{2} t^{1 / 2}}{\nu^{5 / 2}}\left\|(I-M) \mathbb{P}_{\varepsilon} f\right\|_{\infty}^{3}\right) \\
\times\left(\left\|A_{\varepsilon}^{1 / 2} w_{0}\right\|+\frac{t^{1 / 2}}{\nu^{1 / 2}}\left\|(I-M) \mathbb{P}_{\varepsilon} f\right\|_{\infty}\right) .
\end{aligned}
$$

Let us return to inequality (3.18). Note that there is a time $T_{1}=T_{1}(\varepsilon)>0$ such that

$$
\varepsilon^{p} \eta_{3}^{-2} \exp \left(\frac{-\nu C_{5}^{-2} \varepsilon^{-2}}{2} T_{1}\right)=\frac{2 C_{5}^{2} \varepsilon^{2}}{\nu^{2}} \varepsilon^{r} \eta_{4}^{-2}
$$


Indeed, this time $T_{1}$ is given by

$$
T_{1} \stackrel{\text { def }}{=} 2 C_{5}^{2} \varepsilon^{2} \nu^{-1} Q(\varepsilon),
$$

where $Q(\varepsilon)$ is given by (3.6). It follows from (3.18) that if $T_{1}<T^{N}$, then one has

$$
\left\|A_{\varepsilon}^{1 / 2} w(t)\right\|^{2} \leq k_{2}^{2} \varepsilon^{2+r} \eta_{4}^{-2}, \quad T_{1} \leq t<T^{N},
$$

where $k_{2}^{2}=4 C_{5}^{2} \nu^{-2}$.

The next step is to return to (2.23) and the equation satisfied by $v=M u$ : (3.24)

$$
\frac{d v}{d t}+\nu A_{\varepsilon} v=M \mathbb{P}_{\varepsilon} f-M B_{\varepsilon}(v, v)-M B_{\varepsilon}(v, w)-M B_{\varepsilon}(w, v)-M B_{\varepsilon}(w, w)
$$

By taking the scalar product of (3.24) with $A_{\varepsilon} v$ we obtain (3.25)

$$
\frac{1}{2} \frac{d}{d t}\left\|A_{\varepsilon}^{1 / 2} v\right\|^{2}+\nu\left\|A_{\varepsilon} v\right\|^{2} \leq\left|\left\langle M \mathbb{P}_{\varepsilon} f, A_{\varepsilon} v\right\rangle-b_{\varepsilon}\left(v, v, A_{\varepsilon} v\right)-b_{\varepsilon}\left(w, w, A_{\varepsilon} v\right)\right|
$$

since $b_{\varepsilon}\left(v, w, A_{\varepsilon} v\right)=b_{\varepsilon}\left(w, v, A_{\varepsilon} v\right)=0$ from (2.26). By using the Young inequality (3.4) with (3.2) and (3.3) we obtain

$$
\begin{aligned}
\frac{1}{2} \frac{d}{d t}\left\|A_{\varepsilon}^{1 / 2} v\right\|^{2}+\nu\left\|A_{\varepsilon} v\right\|^{2} \leq & \frac{\nu}{2}\left\|A_{\varepsilon} v\right\|^{2}+\frac{1}{2 \nu}\left\|M \mathbb{P}_{\varepsilon} f\right\|_{\infty}^{2} \\
& +C_{1}\|v\|^{1 / 2}\left\|A_{\varepsilon}^{1 / 2} v\right\|\left\|A_{\varepsilon} v\right\|^{3 / 2} \\
& +C_{2} \varepsilon^{1 / 2}\left\|A_{\varepsilon}^{1 / 2} w\right\|^{3 / 2}\left\|A_{\varepsilon} w\right\|^{1 / 2}\left\|A_{\varepsilon} v\right\|,
\end{aligned}
$$

which implies that

$$
\begin{aligned}
\frac{d}{d t}\left\|A_{\varepsilon}^{1 / 2} v\right\|^{2}+\nu\left\|A_{\varepsilon} v\right\|^{2} \leq & \frac{1}{\nu}\left\|M \mathbb{P}_{\varepsilon} f\right\|_{\infty}^{2}+\frac{\nu}{2}\left\|A_{\varepsilon} v\right\|^{2}+\frac{27}{2 \nu^{3}} C_{1}^{4}\|v\|^{2}\left\|A_{\varepsilon}^{1 / 2} v\right\|^{4} \\
& +\frac{\nu}{2}\left\|A_{\varepsilon} v\right\|^{2}+\frac{2}{\nu} C_{2}^{2} \varepsilon\left\|A_{\varepsilon}^{1 / 2} w\right\|^{3}\left\|A_{\varepsilon} w\right\| .
\end{aligned}
$$

Consequently

$$
\begin{aligned}
\frac{d}{d t}\left\|A_{\varepsilon}^{1 / 2} v\right\|^{2} \leq & \left(\frac{27}{2 \nu^{3}} C_{1}^{4}\|v\|^{2}\left\|A_{\varepsilon}^{1 / 2} v\right\|^{2}\right)\left\|A_{\varepsilon}^{1 / 2} v\right\|^{2} \\
& +\frac{1}{\nu}\left\|M \mathbb{P}_{\varepsilon} f\right\|_{\infty}^{2}+\frac{2 C_{2}^{2} \varepsilon}{\nu}\left\|A_{\varepsilon}^{1 / 2} w\right\|^{3}\left\|A_{\varepsilon} w\right\| .
\end{aligned}
$$

By using the Gronwall inequality with (3.28) one finds that

$$
\left\|A_{\varepsilon}^{1 / 2} v(t)\right\|^{2} \leq e^{G(t)}\left(\left\|A_{\varepsilon}^{1 / 2} v_{0}\right\|^{2}+H(t)\right), \quad t \geq 0
$$

where

$$
\begin{gathered}
H(t)=\int_{0}^{t} h(s) d s, \quad G(t)=\int_{0}^{t} g(s) d s, \\
h(t)=\frac{1}{\nu}\left\|M \mathbb{P}_{\varepsilon} f\right\|_{\infty}^{2}+\frac{2 C_{2}^{2} \varepsilon}{\nu}\left\|A_{\varepsilon}^{1 / 2} w\right\|^{3}\left\|A_{\varepsilon} w\right\|, \\
g(t)=\frac{27}{2 \nu^{3}} C_{1}^{4}\|v\|^{2}\left\|A_{\varepsilon}^{1 / 2} v\right\|^{2} .
\end{gathered}
$$


Restricting to $t \leq \min \left(T_{1}, T^{N}\right)$ and using (3.21) we see that (3.33)

$$
\begin{aligned}
\frac{2 C_{2}^{2} \varepsilon}{\nu} \int_{0}^{t}\left\|A_{\varepsilon}^{1 / 2} w\right\|^{3}\left\|A_{\varepsilon} w\right\| d s \leq & D_{2} \varepsilon^{2}\left(\left\|A_{\varepsilon}^{1 / 2} w_{0}\right\|+t^{1 / 2}\left\|(I-M) \mathbb{P}_{\varepsilon} f\right\|_{\infty}\right) \\
& \times\left(\left\|A_{\varepsilon}^{1 / 2} w_{0}\right\|^{3}+\varepsilon^{2} t^{1 / 2}\left\|(I-M) \mathbb{P}_{\varepsilon} f\right\|_{\infty}^{3}\right),
\end{aligned}
$$

where $D_{2}=8 C_{2}^{2} C_{5} \nu^{-2} \max \left(3^{-1 / 2}, 2 C_{5}^{2} \nu^{-5 / 2}\right) \max \left(1, \nu^{-1 / 2}\right)$. By using (3.1) and (3.33) one obtains

$$
H(t) \leq T_{1} \nu^{-1} \eta_{2}^{-2}+D_{2} \varepsilon^{2}\left(\varepsilon^{3 p / 2} \eta_{3}^{-3}+T_{1}^{1 / 2} \varepsilon^{2+3 r / 2} \eta_{4}^{-3}\right)\left(\varepsilon^{p / 2} \eta_{3}^{-1}+T_{1}^{1 / 2} \varepsilon^{r / 2} \eta_{4}^{-1}\right)
$$

for $0 \leq t \leq \min \left(T_{1}, T^{N}\right)$. Consequently from (3.22), the fact that $p \geq-1$, $r>-2$, and the Young inequality (3.4) one deduces that

$$
H(t) \leq E_{1}(\varepsilon)+\frac{7}{4} D_{2} \eta_{3}^{-4}, \quad 0 \leq t \leq \min \left(T_{1}, T^{N}\right),
$$

where

$$
E_{1}(\varepsilon) \stackrel{\text { def }}{=} D_{3}\left(\varepsilon^{2} Q \eta_{2}^{-2}+\varepsilon^{2} Q \eta_{4}^{-4}+\varepsilon^{2} Q^{2} \eta_{4}^{-4}+\varepsilon^{3 / 2} Q^{1 / 2} \eta_{3}^{-1} \eta_{4}^{-3}\right)
$$

and

$$
D_{3} \stackrel{\text { def }}{=} \max \left(2 C_{5}^{2} \nu^{-2}, 2 D_{2} C_{5}^{2} \nu^{-1}, \sqrt{2} D_{2} C_{5} \nu^{-1 / 2}, D_{2} C_{5}^{4} \nu^{-2}\right) \text {. }
$$

By using Hypothesis $\mathrm{H} 1$ we see that

$$
E_{1}(\varepsilon) \rightarrow 0 \quad \text { as } \varepsilon \rightarrow 0^{+} .
$$

It follows from (3.1) and (3.29) that

$$
\left\|A_{\varepsilon}^{1 / 2} v(t)\right\|^{2} \leq e^{G(t)}\left(\eta_{1}^{-2}+E_{1}(\varepsilon)+\frac{7}{4} D_{2} \eta_{3}^{-4}\right), \quad 0 \leq t \leq \min \left(T_{1}, T^{N}\right) .
$$

The next objective is to show that $G(t)$ is small. By taking the scalar product of (2.5) with $u$ and using the fact that $b_{\varepsilon}(u, u, u)=0$, we obtain

$$
\begin{aligned}
\frac{1}{2} \frac{d}{d t}\|u\|^{2}+\nu\left\|A_{\varepsilon}^{1 / 2} u\right\|^{2} & \leq\left|\left\langle\mathbb{P}_{\varepsilon} f, u\right\rangle\right|=\left|\left\langle A_{\varepsilon}^{-1 / 2} \mathbb{P}_{\varepsilon} f, A_{\varepsilon}^{1 / 2} u\right\rangle\right| \\
& \leq \frac{\nu}{2}\left\|A_{\varepsilon}^{1 / 2} u\right\|^{2}+\frac{1}{2 \nu}\left\|A_{\varepsilon}^{-1 / 2} \mathbb{P}_{\varepsilon} f\right\|_{\infty}^{2},
\end{aligned}
$$

which implies that

$$
\begin{gathered}
\|u(t)\|^{2}-\left\|u\left(t_{0}\right)\right\|^{2}+\nu \int_{t_{0}}^{t}\left\|A_{\varepsilon}^{1 / 2} u(s)\right\|^{2} d s \leq \frac{t-t_{0}}{\nu}\left\|A_{\varepsilon}^{-1 / 2} \mathbb{P}_{\varepsilon} f\right\|_{\infty}^{2} \\
\leq \frac{2\left(t-t_{0}\right)}{\nu}\left(\left\|A_{\varepsilon}^{-1 / 2} M \mathbb{P}_{\varepsilon} f\right\|_{\infty}^{2}+\left\|A_{\varepsilon}^{-1 / 2}(I-M) \mathbb{P}_{\varepsilon} f\right\|_{\infty}^{2}\right) .
\end{gathered}
$$

By using (2.6), (2.22), and the Gronwall inequality one finds

$$
\|v(t)\|^{2} \leq\|u(t)\|^{2} \leq\left\|u_{0}\right\|^{2}+2 \lambda_{1}^{-2} \nu^{-2}\left\|M \mathbb{P}_{\varepsilon} f\right\|_{\infty}^{2}+2 C_{5}^{2} \lambda_{1}^{-1} \nu^{-2} \varepsilon^{2}\left\|(I-M) \mathbb{P}_{\varepsilon} f\right\|_{\infty}^{2} .
$$

Since $v_{0}, w_{0} \in D\left(A_{\varepsilon}^{1 / 2}\right)$, one has from (2.6) and (2.22) that

$$
\left\|u_{0}\right\|^{2}=\left\|v_{0}\right\|^{2}+\left\|w_{0}\right\|^{2} \leq \lambda_{1}^{-1}\left\|A_{\varepsilon}^{1 / 2} v_{0}\right\|^{2}+C_{5}^{2} \varepsilon^{2}\left\|A_{\varepsilon}^{1 / 2} w_{0}\right\|^{2} .
$$


Now putting (3.36) and (3.37) together we obtain

$$
\|v(t)\|^{2} \leq D_{4}\left(\left\|A_{\varepsilon}^{1 / 2} v_{0}\right\|^{2}+\varepsilon^{2}\left\|A_{\varepsilon}^{1 / 2} w_{0}\right\|^{2}+\left\|M \mathbb{P}_{\varepsilon} f\right\|_{\infty}^{2}+\varepsilon^{2}\left\|(I-M) \mathbb{P}_{\varepsilon} f\right\|_{\infty}^{2}\right)
$$

where $D_{4}=\max \left(\lambda_{1}^{-1}, C_{5}^{2}, 2 \lambda_{1}^{-2} \nu^{-2}, 2 C_{5}^{2} \lambda_{1}^{-1} \nu^{-2}\right)$. From (3.9) one has

$$
\left\|A_{\varepsilon}^{1 / 2} v(t)\right\|^{2} \leq\left\|A_{\varepsilon}^{1 / 2} u(t)\right\|^{2} \leq N R_{0}^{2}, \quad 0 \leq t<T^{N} .
$$

Next we use (3.1), (3.22), (3.38), and (3.39) to observe that

$$
G(t) \leq E_{2}(\varepsilon), \quad 0 \leq t \leq \min \left(T_{1}, T^{N}\right),
$$

where

$$
E_{2}(\varepsilon) \stackrel{\text { def }}{=} \varepsilon^{2} Q D_{5}\left(\eta_{1}^{-2}+\varepsilon^{2+p} \eta_{3}^{-2}+\eta_{2}^{-2}+\varepsilon^{2+r} \eta_{4}^{-2}\right) N R_{0}^{2}
$$

and $D_{5}=27 C_{1}^{4} C_{5}^{2} \nu^{-4} D_{4}$. After using (3.8) and expanding, one observes that the right-hand side of (3.41) contains 13 terms of the form

$$
c \varepsilon^{b_{0}} \eta_{i}^{-2} \eta_{j}^{-2} \varepsilon^{1 / 4} Q(\varepsilon)
$$

3 terms of the form

$$
c \varepsilon^{3 / 4} \eta_{i}^{-2} \eta_{3}^{-2} \varepsilon^{1 / 4} Q(\varepsilon)
$$

and 4 terms of the form

$$
c \varepsilon^{b_{1}} \eta_{i}^{-2} \eta_{3}^{-4} \varepsilon^{1 / 4} Q(\varepsilon)
$$

where $b_{0} \geq 1, b_{1} \geq \frac{7}{4}, i, j=1,2,3,4$, and where $c$ denotes positive constants which are bounded as $\varepsilon \rightarrow 0^{+}$. By using Hypothesis $\mathrm{H} 1$, it is a straight-forward verification to see that each of these terms goes to 0 as $\varepsilon \rightarrow 0$. In other words, $E_{2}(\varepsilon) \rightarrow 0$ as $\varepsilon \rightarrow 0^{+}$. By combining (3.34) and (3.40) one obtains

$$
\left\|A_{\varepsilon}^{1 / 2} v(t)\right\|^{2} \leq e^{E_{2}(\varepsilon)}\left(\eta_{1}^{-2}+E_{1}(\varepsilon)+\frac{7}{4} D_{2} \eta_{3}^{-4}\right), \quad 0 \leq t \leq \min \left(T_{1}, T^{N}\right),
$$

provided $0<\varepsilon \leq \varepsilon_{2}$.

Now set $N \stackrel{\text { def }}{=} 1+\max \left(4, \frac{7}{2} D_{2}\right)$, where $D_{2}$ is given above, and choose $\varepsilon_{3}$ so that $0<\varepsilon_{3} \leq \varepsilon_{2}(N)$ and

$$
e^{E_{2}(\varepsilon)} \leq 2, \quad E_{1}(\varepsilon) \leq \eta_{1}^{-2}, \quad 2 C_{5}^{2} \varepsilon^{2 / 3} \leq \nu^{2}
$$

for $0<\varepsilon \leq \varepsilon_{3}$.

We claim that for $0<\varepsilon \leq \varepsilon_{3}$ one has $T_{1}<T^{N}$. To prove this we assume on the contrary that $T^{N} \leq T_{1}<\infty$. Then (3.1) and (3.18) imply that

$$
\left\|A_{\varepsilon}^{1 / 2} w\left(T^{N}\right)\right\|^{2} \leq\left\|A_{\varepsilon}^{1 / 2} w_{0}\right\|^{2}+\frac{1}{2} k_{2}^{2} \varepsilon^{2}\left\|(I-M) \mathbb{P}_{\varepsilon} f\right\|_{\infty}^{2} \leq \varepsilon^{p} \eta_{3}^{-2}+\frac{1}{2} k_{2}^{2} \varepsilon^{2+r} \eta_{4}^{-2},
$$

where $k_{2}^{2} \stackrel{\text { def }}{=} 4 C_{5}^{2} \nu^{-2}$. Since $r>-2$, we have $2+r \geq \frac{2}{3}+\frac{r}{3}$, and consequently (3.43) implies that

$$
\left\|A_{\varepsilon}^{1 / 2} w\left(T^{N}\right)\right\|^{2} \leq \varepsilon^{p} \eta_{3}^{-2}+\varepsilon^{r / 3} \eta_{4}^{-2}, \quad 0<\varepsilon \leq \varepsilon_{3} .
$$

On the other hand, (3.42) and (3.43) imply that for $0<\varepsilon \leq \varepsilon_{3}$ one has

$$
\left\|A_{\varepsilon}^{1 / 2} v(t)\right\|^{2} \leq 2\left(2 \eta_{1}^{-2}+\frac{7}{4} D_{2} \eta_{3}^{-4}\right), \quad 0 \leq t \leq \min \left(T_{1}, T^{N}\right) .
$$


By adding (3.44) and (3.45) we obtain

$$
\left\|A_{\varepsilon}^{1 / 2} u\left(T^{N}\right)\right\|^{2} \leq\left(4 \eta_{1}^{-2}+\frac{7}{2} D_{2} \eta_{3}^{-4}+\varepsilon^{p} \eta_{3}^{-2}+\varepsilon^{r / 3} \eta_{4}^{-2}\right)<N R_{0}^{2}, \quad 0<\varepsilon \leq \varepsilon_{3},
$$

which contradicts (3.10). Hence one has $T_{1}<T^{N}$ for $0<\varepsilon \leq \varepsilon_{3}$.

Finally we set $k_{1}^{2} \stackrel{\text { def }}{=} \frac{7}{2} D_{2}$ and $\varepsilon_{1} \stackrel{\text { def }}{=} \varepsilon_{3}$. It then follows from (3.45) that

and from (3.23) that

$$
\left\|A_{\varepsilon}^{1 / 2} v\left(T_{1}\right)\right\|^{2} \leq 4 \eta_{1}^{-2}+k_{1}^{2} \eta_{3}^{-4}
$$

which completes the proof of Lemma 3.1 .

$$
\left\|A_{\varepsilon}^{1 / 2} w\left(T_{1}\right)\right\|^{2} \leq k_{2}^{2} \varepsilon^{2+r} \eta_{4}^{-2}
$$

Remark. The proof of Lemma 3.1 still works if we take $r=-2$. However, the result of Lemma 3.1 is interesting only in the case where $\varepsilon^{2+r} \eta_{4}^{-2}$ is bounded as $\varepsilon \rightarrow 0^{+}$; see also Hypothesis $\mathrm{H} 2(\mathrm{a}, \mathrm{b})$ given below. If $\eta_{4}^{-2} \rightarrow \infty$ as $\varepsilon \rightarrow 0^{+}$, this implies that $r$ must satisfy $r>-2$. In Theorems 7, 8, and 9 we will impose a stronger requirement, viz. that $\varepsilon^{2+r} \eta_{4}^{-2} \rightarrow 0$ as $\varepsilon \rightarrow 0^{+}$. This is the reason why we impose the requirement that $r>-2$ in Hypothesis $\mathrm{H} 1$.

3.2. Strategy of proof. The argument of Lemma 3.1 can of course be repeated with the new initial conditions satisfying (3.7) instead of (3.1). By making the realistic assumption that

$$
k_{2}^{2} \varepsilon^{2+r} \eta_{4}^{-2} \leq \varepsilon^{p} \eta_{3}^{-2}
$$

one needs only to replace $\eta_{1}^{-2}$ with $\left(4 \eta_{1}^{-2}+k_{1}^{2} \eta_{3}^{-4}\right)$, and the entire argument carries through. Unfortunately, this is not a good strategy, because one is forced to choose a smaller value for $\varepsilon_{2}$, and thereby a smaller value for $\varepsilon_{1}$. It is important to take advantage of the fact that $k_{2}^{2} \varepsilon^{2+r} \eta_{4}^{-2}$ can be made small instead of using the crude bound (3.46). As a result of Lemma 3.1 we can now assume the initial condition $\left\|A_{\varepsilon}^{1 / 2} w_{0}\right\|^{2}$ to be small for $0<\varepsilon \leq \varepsilon_{1}$.

For the Long Time Argument we begin by assuming $\left\|A_{\varepsilon}^{1 / 2} w_{0}\right\|^{2}$ is small, i.e., $\left\|A_{\varepsilon}^{1 / 2} w_{0}\right\|^{2} \leq k_{2}^{2} \varepsilon^{2+r} \eta_{4}^{-2}$ and $\left\|A_{\varepsilon}^{1 / 2} v_{0}\right\|^{2} \leq 4 \eta_{1}^{-2}+k_{1}^{2} \eta_{3}^{-4}$. In the course of the argument we show that if $\varepsilon$ is sufficiently small, then the dilated 3D Navier-Stokes evolutionary equation $(2.5)$ has a strong solution on a suitable interval $\left[0,2 T_{0}\right]$ where $T_{0}=T_{0}(\varepsilon)$ is finite but large. We also show that $\left\|A_{\varepsilon}^{1 / 2} w(t)\right\|^{2} \leq k_{2}^{2} \varepsilon^{2+r} \eta_{4}^{-2}$ and $\left\|A_{\varepsilon}^{1 / 2} v(t)\right\|^{2} \leq \frac{1}{2}\left(4 \eta_{1}^{-2}+k_{1}^{2} \eta_{3}^{-4}\right)$ on the halfinterval $t \in\left[T_{0}, 2 T_{0}\right]$. This, of course, permits one to prove the $H^{1}$-Regularity Theorem by using the Long Time Argument with induction.

3.3. Long-Time argument. In this section we continue the analysis of the ShortTime Argument. The terms $\eta_{i}(\varepsilon), 1 \leq i \leq 4, r$, and $p$ will be assumed to satisfy Hypothesis H1. In addition, we will assume that the following Hypothesis H2(a, b) is satisfied, where $a$ and $b$ are sufficiently large:

(1) Let $a>0$ be fixed. Then one has

$$
\left\{\begin{array}{l}
\varepsilon^{5 / 8} \eta^{-2} \exp \left(a \eta^{-4}\right) \rightarrow 0 \\
\eta^{-2} \rightarrow \infty
\end{array}\right.
$$


as $\varepsilon \rightarrow 0^{+}$, where

$$
\eta^{-2} \stackrel{\text { def }}{=} \max \left(4 \eta_{1}^{-2}+k_{1}^{2} \eta_{3}^{-4}+k_{2}^{2} \varepsilon^{2+r} \eta_{4}^{-2}, 1\right)
$$

are $\varepsilon^{5 / 8} \exp \left(2 a \eta_{2}^{-4}\right)$ is bounded for $0<\varepsilon \leq 1$. (The constants $k_{1}$ and $k_{2}$ are given in Lemma 3.1.)

(2) Let $b>0$ be fixed. Then for any $\lambda, 0<\lambda<1$, there is an $\varepsilon_{4}=$ $\varepsilon_{4}(b, \lambda)>0$ such that one has

$$
\eta_{2}^{-2} \exp \left(b \eta_{2}^{-4}\right) \leq \lambda\left(4 \eta_{1}^{-2}+k_{1}^{2} \eta_{3}^{-4}\right), \quad 0<\varepsilon<\varepsilon_{4} .
$$

(3) The function $\varepsilon^{4+2 r} \eta_{4}^{-4}\left(\log \eta^{-4}+1\right)$ is bounded as $\varepsilon \rightarrow 0^{+}$. Our objective now is to prove the following result:

Lemma 3.2. Assume that both Hypotheses $H 1$ and $H 2(a, b)$ are satisfied, where $a$ and $b$ are sufficiently large. Then there is an $\varepsilon_{0}>0$ such that for every $\varepsilon$, $0<\varepsilon \leq \varepsilon_{0}$, there is a time $T_{0}=T_{0}(\varepsilon)>0$ with the property that, for $0<\varepsilon \leq \varepsilon_{0}$, whenever the initial conditions

$$
\begin{cases}\left\|A_{\varepsilon}^{1 / 2} v_{0}\right\|^{2} \leq 4 \eta_{1}^{-2}+k_{1}^{2} \eta_{3}^{-4}, & \left\|M \mathbb{P}_{\varepsilon} f\right\|_{\infty}^{2} \leq \eta_{2}^{-2}, \\ \left\|A_{\varepsilon}^{1 / 2} w_{0}\right\|^{2} \leq k_{2}^{2} \varepsilon^{2+r} \eta_{4}^{-2}, & \left\|(I-M) \mathbb{P}_{\varepsilon} f\right\|_{\infty}^{2} \leq \varepsilon^{r} \eta_{4}^{-2}\end{cases}
$$

are satisfied, then the solution $u(t)$ of (2.5) satisfies $u(t) \in D\left(A_{\varepsilon}^{1 / 2}\right)$ for $0 \leq t \leq$ $2 T_{0}$ and

$$
\left\{\begin{array}{l}
\left\|A_{\varepsilon}^{1 / 2} v(t)\right\|^{2} \leq \frac{1}{2}\left(4 \eta_{1}^{-2}+k_{1}^{2} \eta_{3}^{-4}\right) \\
\left\|A_{\varepsilon}^{1 / 2} w(t)\right\|^{2} \leq k_{2}^{2} \varepsilon^{2+r} \eta_{4}^{-2}
\end{array}\right.
$$

for $T_{0} \leq t \leq 2 T_{0}$.

Proof. The proof begins as in Lemma 3.1. For any positive numbers $d_{1}$ and $d_{2}$ we define $R_{0}^{2}=R_{0}^{2}\left(\varepsilon, d_{1}, d_{2}\right)$ by

$$
R_{0}^{2}=1+\left(\eta^{-2}+\eta_{2}^{-2}+d_{1}\right)\left[1+\exp \left(d_{2} \eta^{-4}\right) \exp \left(2 d_{2} \eta_{2}^{-4}\right)\right]
$$

The values of $d_{1}$ and $d_{2}$ will be fixed later. Note that $R_{0}^{2} \geq \eta^{-2} \geq\left\|A_{\varepsilon}^{1 / 2} u_{0}\right\|^{2}$. Therefore, it follows from Lemma 3.0 that for any $N>1$ there is a time $T^{N}$, $0<T^{N} \leq \infty$, such that

$$
\left\|A_{\varepsilon}^{1 / 2} u(t)\right\|^{2} \leq N R_{0}^{2}, \quad 0 \leq t<T^{N}
$$

Without loss of generality we let $\left[0, T^{N}\right)$ denote the maximal time interval for which (3.50) is valid. Therefore if $T^{N}<\infty$ one must have

$$
\left\|A_{\varepsilon}^{1 / 2} u\left(T^{N}\right)\right\|^{2}=N R_{0}^{2} .
$$

By taking the scalar product of the $w$-equation (3.11) with $A_{\varepsilon} w$, we then obtain (3.13) with the same value for $D_{1}$. For $0 \leq t<T^{N}$ one has

$$
D_{1}^{2} \varepsilon^{5 / 4}\left\|A_{\varepsilon}^{1 / 2} u\right\|^{2} \leq D_{1}^{2} N \varepsilon^{5 / 4} R_{0}^{2}
$$


where $R_{0}^{2}$ is given by (3.49). From Hypotheses $\mathrm{H} 1$ and $\mathrm{H} 2(\mathrm{a}, \mathrm{b})$, the righthand side of (3.52) goes to 0 as $\varepsilon \rightarrow 0^{+}$, provided $a \geq d_{2}$ and $b \geq 2 d_{2}$. Consequently there is an $\varepsilon_{5}=\varepsilon_{5}\left(N, d_{1}, d_{2}\right), 0<\varepsilon_{5} \leq \varepsilon_{1}$, where $\varepsilon_{1}$ is given by Lemma 3.1, such that

$$
D_{1} N^{1 / 2} \varepsilon^{5 / 8} R_{0} \leq \nu / 2, \quad 0<\varepsilon \leq \varepsilon_{5} .
$$

As a result, (3.16), (3.17), (3.18), and (3.19) are valid for $0 \leq t<T^{N}$ and $0<\varepsilon \leq \varepsilon_{5}$. We now restrict to $0<\varepsilon \leq \varepsilon_{5}$ for the remainder of the argument. By using (3.47) we see that for $0<\varepsilon \leq \varepsilon_{5}$ inequality (3.18) now assumes the form

$$
\left\|A_{\varepsilon}^{1 / 2} w(t)\right\|^{2} \leq\left[k_{2}^{2} \exp \left(\frac{-\nu C_{5}^{-2} \varepsilon^{-2}}{2} t\right)+\frac{1}{2} k_{2}^{2}\right] \varepsilon^{2+r} \eta_{4}^{-2} \leq \frac{3}{2} k_{2}^{2} \varepsilon^{2+r} \eta_{4}^{-2}
$$

for $0 \leq t<T^{N}$. By using (3.47) once again, (3.19) becomes

$$
\begin{aligned}
\int_{0}^{t}\left\|A_{\varepsilon} w(s)\right\|^{2} d s & \leq \frac{2}{\nu}\left\|A_{\varepsilon}^{1 / 2} w_{0}\right\|^{2}+\frac{2 t}{\nu^{2}}\left\|(I-M) \mathbb{P}_{\varepsilon} f\right\|_{\infty}^{2} \\
& \leq D_{9}^{2}\left(\varepsilon^{2}+t\right) \varepsilon^{r} \eta_{4}^{-2}
\end{aligned}
$$

for $0 \leq t<T^{N}$, where $D_{9}^{2}=\max \left(2 k_{2}^{2} \nu^{-1}, 2 \nu^{-2}\right)$. In addition, by integrating (3.16) and using (3.53) we have for $0<\varepsilon \leq \varepsilon_{5} \leq 1$

$$
\int_{t-1}^{t}\left\|A_{\varepsilon} w\right\|^{2} d s \leq \frac{2}{\nu}\left\|A_{\varepsilon}^{1 / 2} w(t-1)\right\|^{2}+\frac{2}{\nu^{2}}\left\|(I-M) \mathbb{P}_{\varepsilon} f\right\|_{\infty}^{2} \leq D_{10}^{2} \varepsilon^{r} \eta_{4}^{-2},
$$

for $1 \leq t<T^{N}$, where $D_{10}^{2}=3 k_{2}^{2} \nu^{-1}+2 \nu^{-2}$. It follows from (3.5) and (3.53) that

$$
\begin{aligned}
\int_{0}^{t}\left\|A_{\varepsilon}^{1 / 2} w\right\|^{6} d s & \leq 4 \int_{0}^{t}\left[k_{2}^{6} \exp \left(\frac{-3 \nu C_{5}^{-2} \varepsilon^{-2}}{2} s\right)+\frac{1}{8} k_{2}^{6}\right] \varepsilon^{6+3 r} \eta_{4}^{-6} d s \\
& \leq D_{11}^{2}\left(\varepsilon^{2}+t\right) \varepsilon^{6+3 r} \eta_{4}^{-6}
\end{aligned}
$$

for $0 \leq t<T^{N}$, where $D_{11}^{2}=4 k_{2}^{6} \max \left(2 C_{5}^{2}(3 \nu)^{-1}, \frac{1}{8}\right)$. Using $0<\varepsilon \leq 1$ one has

$$
\int_{t-1}^{t}\left\|A_{\varepsilon}^{1 / 2} w\right\|^{6} d s \leq 2 D_{11}^{2} \varepsilon^{6+3 r} \eta_{4}^{-6}
$$

for $1 \leq t<T^{N}$. Using the argument in (3.21) one then obtains

$$
\int_{0}^{t}\left\|A_{\varepsilon}^{1 / 2} w\right\|^{3}\left\|A_{\varepsilon} w\right\| d s \leq D_{9} D_{11}\left(\varepsilon^{2}+t\right) \varepsilon^{3+2 r} \eta_{4}^{-4}, \quad 0 \leq t<T^{N},
$$

and, since $0<\varepsilon \leq 1$,

$$
\int_{t-1}^{t}\left\|A_{\varepsilon}^{1 / 2} w\right\|^{3}\left\|A_{\varepsilon} w\right\| d s \leq 2 D_{10} D_{11} \varepsilon^{3+2 r} \eta_{4}^{-4}, \quad 1 \leq t<T^{N} .
$$

Next we return to the $v$-equation (3.24). By taking the scalar product of (3.24) with $v$ and using $b_{\varepsilon}(v, v, v)=0$ together with (2.26) and $(3.3)$ we 
obtain

$$
\begin{aligned}
\frac{1}{2} \frac{d}{d t}\|v\|^{2}+\lambda_{1} \nu\|v\|^{2} & \leq \frac{1}{2} \frac{d}{d t}\|v\|^{2}+\nu\left\|A_{\varepsilon}^{1 / 2} v\right\|^{2} \\
& \leq\left(\left\|M \mathbb{P}_{\varepsilon} f\right\|_{\infty}+C_{2} \varepsilon^{1 / 2}\left\|A_{\varepsilon}^{1 / 2} w\right\|^{3 / 2}\left\|A_{\varepsilon} w\right\|^{1 / 2}\right)\|v\| \\
& \leq \lambda_{1}^{-1 / 2}\left(\left\|M \mathbb{P}_{\varepsilon} f\right\|_{\infty}+C_{2} \varepsilon^{1 / 2}\left\|A_{\varepsilon}^{1 / 2} w\right\|^{3 / 2}\left\|A_{\varepsilon} w\right\|^{1 / 2}\right)\left\|A_{\varepsilon}^{1 / 2} v\right\| .
\end{aligned}
$$

By using the Young inequality we get

$$
\frac{d}{d t}\|v\|^{2}+\lambda_{1} \nu\|v\|^{2} \leq \frac{1}{\lambda_{1} \nu}\left(\left\|M \mathbb{P}_{\varepsilon} f\right\|_{\infty}^{2}+C_{2}^{2} \varepsilon\left\|A_{\varepsilon}^{1 / 2} w\right\|^{3}\left\|A_{\varepsilon} w\right\|\right)
$$

and

$$
\frac{d}{d t}\|v\|^{2}+\nu\left\|A_{\varepsilon}^{1 / 2} v\right\|^{2} \leq \frac{1}{\lambda_{1} \nu}\left(\left\|M \mathbb{P}_{\varepsilon} f\right\|_{\infty}^{2}+C_{2}^{2} \varepsilon\left\|A_{\varepsilon}^{1 / 2} w\right\|^{3}\left\|A_{\varepsilon} w\right\|\right) .
$$

By using the Gronwall inequality for (3.56) one finds that

$$
\begin{aligned}
\|v(t)\|^{2} \leq & e^{-\nu \lambda_{1} t}\left\|v_{0}\right\|^{2}+\lambda_{1}^{-2} \nu^{-2}\left\|M \mathbb{P}_{\varepsilon} f\right\|_{\infty}^{2} \\
& +\lambda_{1}^{-1} \nu^{-1} C_{2}^{2} \varepsilon \int_{0}^{t}\left\|A_{\varepsilon}^{1 / 2} w\right\|^{3}\left\|A_{\varepsilon} w\right\| d s
\end{aligned}
$$

for $0 \leq t<T^{N}$. Next by using (2.6), (3.47), and (3.54) we find

$$
\|v(t)\|^{2} \leq D_{12} \gamma(\varepsilon, t), \quad 0 \leq t<T^{N},
$$

where $D_{12}=\max \left(\lambda_{1}^{-1}, \lambda_{1}^{-2} \nu^{-2}, \lambda_{1}^{-1} \nu^{-1} C_{2}^{2} D_{9} D_{11}\right)$ and

$$
\gamma=\gamma(\varepsilon, t) \stackrel{\text { def }}{=}\left(e^{-\nu \lambda_{1} t} \eta^{-2}+\eta_{2}^{-2}+\left[\varepsilon^{2}+t\right] \varepsilon^{4+2 r} \eta_{4}^{-4}\right) \text {. }
$$

Similarly by integrating (3.57) and using (3.47) and (3.54) we obtain

$$
\begin{aligned}
\int_{0}^{t}\left\|A_{\varepsilon}^{1 / 2} v\right\|^{2} d s \leq & \nu^{-1}\left\|v_{0}\right\|^{2}+\lambda_{1}^{-1} \nu^{-2} t\left\|M \mathbb{P}_{\varepsilon} f\right\|_{\infty}^{2} \\
& +\lambda_{1}^{-1} \nu^{-2} C_{2}^{2} \varepsilon \int_{0}^{t}\left\|A_{\varepsilon}^{1 / 2} w\right\|^{3}\left\|A_{\varepsilon} w\right\| d s \\
\leq & \lambda_{1}^{-1} \nu^{-1}\left(4 \eta_{1}^{-2}+k_{1}^{2} \eta_{3}^{-4}\right)+\lambda_{1}^{-1} \nu^{-2} t \eta_{2}^{-2} \\
& +\lambda_{1}^{-1} \nu^{-2} C_{2}^{2} D_{9} D_{11}\left(\varepsilon^{2}+t\right) \varepsilon^{4+2 r} \eta_{4}^{-4}
\end{aligned}
$$

for $0 \leq t<T^{N}$. It follows that

$$
\int_{0}^{t}\left\|A_{\varepsilon}^{1 / 2} v\right\|^{2} d s \leq D_{13} \gamma(\varepsilon, t), \quad 0 \leq t<\min \left(1, T^{N}\right),
$$

where $D_{13}=\max \left(\lambda_{1}^{-1} \nu^{-1} e^{\nu \lambda_{1}}, \lambda_{1}^{-1} \nu^{-2}, \lambda_{1}^{-1} \nu^{-2} C_{2}^{2} D_{9} D_{11}\right)$. Furthermore, by integrating (3.57) once again we find

$$
\begin{aligned}
\int_{t-1}^{t}\left\|A_{\varepsilon}^{1 / 2} v\right\|^{2} d s \leq & \nu^{-1}\|v(t-1)\|^{2}+\lambda_{1}^{-1} \nu^{-2}\left\|M \mathbb{P}_{\varepsilon} f\right\|_{\infty}^{2} \\
& +\lambda_{1}^{-1} \nu^{-2} C_{2}^{2} \varepsilon \int_{t-1}^{t}\left\|A_{\varepsilon}^{1 / 2} w\right\|^{3}\left\|A_{\varepsilon} w\right\| d s
\end{aligned}
$$


From (3.47), (3.55), (3.59), and (3.60) we get

$$
\int_{t-1}^{t}\left\|A_{\varepsilon}^{1 / 2} v\right\|^{2} d s \leq D_{14} \gamma(\varepsilon, t), \quad 1 \leq t<T^{N},
$$

where

$$
D_{14}=\nu^{-1} \max \left(D_{12}+\lambda_{1}^{-1} \nu^{-1}, D_{12} e^{\nu \lambda_{1}}+2 D_{10} D_{11} \lambda_{1}^{-1} \nu^{-1} C_{2}^{2}\right) .
$$

By combining (3.59), (3.60), (3.62), and (3.64) we find

$$
\begin{aligned}
\int_{0}^{t}\|v\|^{2}\left\|A_{\varepsilon}^{1 / 2} v\right\|^{2} d s & \leq \sup _{0 \leq s \leq t}\|v(s)\|^{2} \int_{0}^{t}\left\|A_{\varepsilon}^{1 / 2} v\right\|^{2} d s \\
& \leq e^{\nu \lambda_{1}} D_{12} D_{13} \gamma(\varepsilon, t)^{2}
\end{aligned}
$$

for $0 \leq t<\min \left(1, T^{N}\right)$ and

$$
\int_{t-1}^{t}\|v\|^{2}\left\|A_{\varepsilon}^{1 / 2} v\right\|^{2} d s \leq e^{\nu \lambda_{1}} D_{12} D_{14} \gamma(\varepsilon, t)^{2}, \quad 1 \leq t<T^{N} .
$$

Next by taking the scalar product of (3.24) with $A_{\varepsilon} v$ we obtain (3.25), (3.26) (3.27), and (3.28). For $0 \leq t<\min \left(1, T^{N}\right)$ we apply the Gronwall inequality to $(3.28)$ to obtain

$$
\left\|A_{\varepsilon}^{1 / 2} v(t)\right\|^{2} \leq e^{G(t)}\left(\left\|A_{\varepsilon}^{1 / 2} v_{0}\right\|^{2}+H(t)\right), \quad 0 \leq t<\min \left(1, T^{N}\right),
$$

where $H(t)$ and $G(t)$ are given by (3.30), (3.31), and (3.32). From (3.47), (3.54), and (3.65) we find

$$
\left\|A_{\varepsilon}^{1 / 2} v(t)\right\|^{2} \leq D_{16} \gamma(\varepsilon, t) \exp \left(D_{17} \gamma(\varepsilon, t)^{2}\right), \quad 0 \leq t<\min \left(1, T^{N}\right),
$$

where

$$
D_{16}=\max \left(e^{\nu \lambda_{1}}, \nu^{-1}, 2 \nu^{-1} C_{2}^{2} D_{9} D_{11}\right) \quad \text { and } \quad D_{17}=2^{-1} 27 \nu^{-3} C_{1}^{4} e^{\nu \lambda_{1}} D_{12} D_{13} .
$$

For $1 \leq t<T^{N}$ we use the uniform Gronwall inequality (see Foias, et al. $(1987))^{5}$ on $(3.28)$ to obtain

$$
\left\|A_{\varepsilon}^{1 / 2} v(t)\right\|^{2} \leq\left(\int_{t-1}^{t}\left\|A_{\varepsilon}^{1 / 2} v\right\|^{2} d s+\int_{t-1}^{t} h(s) d s\right) \exp \left(\int_{t-1}^{t} g(s) d s\right)
$$

where $h$ and $g$ are given by (3.31) and (3.32). For $t \geq 1$ we use (3.55), (3.66), and (3.64) to derive an inequality similar to (3.67). This can be combined with (3.67) to obtain

$$
\left\|A_{\varepsilon}^{1 / 2} v(t)\right\|^{2} \leq D_{18} \gamma(\varepsilon, t) \exp \left(D_{19} \gamma(\varepsilon, t)^{2}\right), \quad 0 \leq t<T^{N},
$$

${ }^{5}$ Let $y, g, h$ be nonnegative locally integrable functions on $(0, \infty)$, where $y$ is absolutely continuous on $(0, \infty)$, and which satisfy $y^{\prime} \leq g y+h, 0<t<\infty$. Then one has

$$
y(t) \leq\left(\frac{1}{t-\tau} \int_{\tau}^{t} y(s) d s+\int_{\tau}^{t} h(s) d s\right) \exp \left(\int_{\tau}^{t} g(s) d s\right), \quad 0<t<\infty,
$$

where $\tau=\max (0, t-1)$. 
where

$$
\left\{\begin{array}{l}
D_{18}=\max \left(D_{16}, D_{14}+\max \left(\nu^{-1}, 4 C_{2}^{2} D_{10} D_{11} \nu^{-1}\right)\right), \\
D_{19}=27 C_{1}^{4} e^{\nu \lambda_{1}} 2^{-1} \nu^{-3} \max \left(D_{13}, D_{14}\right) D_{12} .
\end{array}\right.
$$

The next step is to define $T_{0}=T_{0}(\varepsilon)$. Since $\eta^{-4} \rightarrow \infty$ as $\varepsilon \rightarrow 0^{+}$, there is no loss in generality in assuming that

$$
2 D_{19} \eta^{-4}>1 \text {. }
$$

We then define $T_{0}$ by requiring

$$
2 D_{19} e^{-2 \nu \lambda_{1} t} \eta^{-4} \leq 1, \quad T_{0} \leq t
$$

that is, set

$$
T_{0} \stackrel{\text { def }}{=} \frac{1}{2 \nu \lambda_{1}} \log \left(2 D_{19} \eta^{-4}\right)
$$

Also define

$$
E_{3}(\varepsilon) \stackrel{\text { def }}{=}\left(\varepsilon^{2}+2 T_{0}(\varepsilon)\right) \varepsilon^{4+2 r} \eta_{4}^{-4} .
$$

It follows from Hypothesis $\mathrm{H} 2(\mathrm{a}, \mathrm{b})$ that there are constants $D_{20}$ and $D_{21}$ such that

$$
E_{3}(\varepsilon) \leq D_{20}, \quad \frac{3}{2} k_{2}^{2} \varepsilon^{2+r} \eta_{4}^{-2} \leq D_{21}
$$

for $0<\varepsilon \leq 1$. The term $R_{0}^{2}=R_{0}^{2}\left(\varepsilon, d_{1}, d_{2}\right)$ is now fixed so that

$$
R_{0}^{2}=R_{0}^{2}\left(\varepsilon, D_{20}, 2 D_{19}\right),
$$

and the term $N$ is fixed so that

$$
N>\max \left(1, D_{21}, D_{22}\right),
$$

where $D_{22}=D_{18} \exp \left(4 D_{19} D_{20}^{2}\right)$. Finally we fix $\varepsilon_{5}=\varepsilon_{5}\left(N, D_{20}, 2 D_{19}\right)$ for these choices of $N, d_{1}$, and $d_{2}$. Furthermore, we require that the constants $a$ and $b$ in Hypothesis $\mathrm{H} 2(\mathrm{a}, \mathrm{b})$ satisfy $a \geq 2 D_{19}$ and $b \geq 4 D_{19}$.

Let us return to the function $\gamma$, which we will write as

$$
\gamma=\gamma(\varepsilon, t)=e^{-\nu \lambda_{1} t} \eta^{-2}+\beta
$$

where

Note that

$$
\beta=\beta(\varepsilon, t) \stackrel{\text { def }}{=} \eta_{2}^{-2}+\left(\varepsilon^{2}+t\right) \varepsilon^{4+2 r} \eta_{4}^{-4}
$$

$$
\gamma^{2} \leq 2 e^{-2 \nu \lambda_{1} t} \eta^{-4}+2 \beta^{2}
$$

and from (3.72) and (3.73) we have

$$
\beta(\varepsilon, t) \leq \eta_{2}^{-2}+D_{20}, \quad 0 \leq t \leq 2 T_{0} .
$$

From (3.70) we see that

$$
D_{18} \gamma \exp \left(D_{19} \gamma^{2}\right) \leq D_{22}\left(e^{-\nu \lambda_{1} t} \eta^{-2}+\eta_{2}^{-2}+D_{20}\right) \exp \left(4 D_{19} \eta_{2}^{-4}+1\right)
$$

for $T_{0} \leq t \leq 2 T_{0}$. 
From (3.53) and (3.73) we find

$$
\left\|A_{\varepsilon}^{1 / 2} w(t)\right\|^{2} \leq D_{21}, \quad 0 \leq t \leq T^{N} .
$$

By using (3.69), (3.76), and (3.77) we find that

$$
\left\|A_{\varepsilon}^{1 / 2} v(t)\right\|^{2} \leq D_{22}\left(e^{-\nu \lambda_{1} t} \eta^{-2}+\eta_{2}^{-2}+D_{20}\right) \exp \left(2 D_{19} \eta^{-4}\right) \exp \left(4 D_{19} \eta_{2}^{-4}\right)
$$

for $0 \leq t \leq \min \left(2 T_{0}, T^{N}\right)$.

We claim that $2 T_{0} \leq T^{N}$. In order to prove this, we assume on the contrary that $T^{N}<2 T_{0}$. From the Pythagorean relation (2.21) and (3.79) and (3.80) it follows that

$$
\left\|A_{\varepsilon}^{1 / 2} u\left(T^{N}\right)\right\|^{2} \leq D_{21}+D_{22}\left(\eta^{-2}+\eta_{2}^{-2}+D_{20}\right) \exp \left(2 D_{19} \eta^{-4}\right) \exp \left(4 D_{19} \eta_{2}^{-4}\right) .
$$

From the definition of $R_{0}^{2}$ in (3.49) and (3.74) and the characterization of $N$ in (3.75) we obtain

$$
\left\|A_{\varepsilon}^{1 / 2} u\left(T^{N}\right)\right\|^{2}<N R_{0}^{2},
$$

which contradicts (3.51). Hence one has $2 T_{0} \leq T^{N}$.

Finally we turn to the verification of (3.48). For this purpose we restrict $t$ to the interval $\left[T_{0}, 2 T_{0}\right]$. From (3.53) we have

$$
\left\|A_{\varepsilon}^{1 / 2} w(t)\right\|^{2} \leq\left[k_{2}^{2} \exp \left(\frac{-\nu C_{5}^{-2} \varepsilon^{-2}}{2} T_{0}\right)+\frac{1}{2} k_{2}^{2}\right] \varepsilon^{2+r} \eta_{4}^{-2}, \quad T_{0} \leq t \leq 2 T_{0} .
$$

From the definition of $T_{0}$ in (3.71), we see that there is an $\varepsilon_{6}, 0<\varepsilon_{6} \leq \varepsilon_{5}$, such that

$$
2 \nu^{-1} C_{5}^{2} \varepsilon^{2} \log 2 \leq T_{0}(\varepsilon), \quad 0<\varepsilon \leq \varepsilon_{6} .
$$

Now (3.81) implies that $\exp \left(-\nu C_{5}^{-2} \varepsilon^{-2} T_{0} / 2\right) \leq \frac{1}{2}$, and consequently,

$$
\left\|A_{\varepsilon}^{1 / 2} w(t)\right\|^{2} \leq k_{2}^{2} \varepsilon^{2+r} \eta_{4}^{-2}, \quad T_{0} \leq t \leq 2 T_{0},
$$

for $0<\varepsilon \leq \varepsilon_{6}$. From (3.69), (3.70), and (3.78) one finds

$$
\left\|A_{\varepsilon}^{1 / 2} v(t)\right\|^{2} \leq \Gamma\left(\eta_{2}^{-2}\right), \quad T_{0} \leq t \leq 2 T_{0},
$$

where

$$
\Gamma(r) \stackrel{\text { def }}{=} D_{22}\left(r+D_{24}\right) \exp \left(4 D_{19} r^{2}+1\right)
$$

where $D_{24}=\left(2 D_{19}\right)^{-1 / 2}+D_{20}$. From Hypothesis $\mathrm{H} 2(\mathrm{a}, \mathrm{b})$ we see that there is an $\varepsilon_{7}, 0<\varepsilon_{7} \leq \varepsilon_{6}$, such that for $0<\varepsilon \leq \varepsilon_{7}$ one has

$$
\Gamma\left(\eta_{2}^{-2}\right) \leq \frac{1}{2}\left(4 \eta_{1}^{-2}+k_{1}^{2} \eta_{3}^{-4}\right), \quad 0<\varepsilon \leq \varepsilon_{7} .
$$

It then follows that for $0<\varepsilon \leq \varepsilon_{7}$ one has

$$
\left\|A_{\varepsilon}^{1 / 2} v(t)\right\|^{2} \leq \frac{1}{2}\left(4 \eta_{1}^{-2}+k_{1}^{2} \eta_{3}^{-4}\right), \quad T_{0} \leq t \leq 2 T_{0} .
$$

By setting $\varepsilon_{0}=\varepsilon_{7}$, we complete the proof of the lemma. 
Proof of Theorem 1. Since $0<\varepsilon_{0} \leq \varepsilon_{1}$, where $\varepsilon_{1}$ and $\varepsilon_{0}$ are given by Lemmas 3.1 and 3.2, the proof of Theorem 1, for $0<\varepsilon \leq \varepsilon_{0}$, now follows by first applying Lemma 3.1 and then using Lemma 3.2 with induction, with $\widehat{T}_{1}=$ $T_{0}+T_{1}$. The estimate for $L_{5}^{2}$ appearing in (2.33) follows from the Pythagorean identity (2.21) together with (3.82) and (3.83). The fact that $u(\cdot)$ belongs to $C^{0}\left([0, \infty), V_{\varepsilon}^{1}\right)$ is now a direct consequence of the local result contained in Lemma 3.0.

\section{4. $H^{2}$-Regularity: TheOREMS 2 AND 3}

In this section, we will prove Theorems 2 and 3.

Proof of Theorem 2. It is known that if $u_{0}$ belongs to $V_{\varepsilon}^{2}=D\left(A_{\varepsilon}\right)$ and $\mathbb{P}_{\varepsilon} f$ belongs to $C^{0}\left([0, \infty), H_{\varepsilon}\right) \cap W^{1, \infty}\left((0, \infty), D\left(A_{\varepsilon}^{-1 / 2}\right)\right)$, then $u$ is in the space $C^{0}\left([0, \infty), V_{\varepsilon}^{2}\right)$, and the time-derivative $u^{\prime}$ belongs to $C^{0}\left([0, \infty) ; H_{\varepsilon}\right)$ and is the solution of the equation

$$
d u^{\prime} / d t+\nu A_{\varepsilon} u^{\prime}+B_{\varepsilon}\left(u^{\prime}, u\right)+B_{\varepsilon}\left(u, u^{\prime}\right)=\mathbb{P}_{\varepsilon} f^{\prime}
$$

with initial condition

$$
u^{\prime}(0)=\mathbb{P}_{\varepsilon} f(0)-B_{\varepsilon}\left(u_{0}, u_{0}\right)-\nu A_{\varepsilon} u_{0}
$$

see Temam $(1982,1983)$. On the other hand, if $u_{0}$ belongs only to $V_{\varepsilon}^{1}$, we will show that one can choose $t_{0}>0$, arbitrarily close to 0 , such that $u\left(t_{0}\right)$ belongs to $D\left(A_{\varepsilon}\right)$. It then follows that $u^{\prime}\left(t_{0}\right)$ belongs to $H_{\varepsilon}$ for every such $t_{0}$, that $u$ belongs to $C^{0}\left(\left[t_{0}, \infty\right), V_{\varepsilon}^{2}\right)$, and that $u^{\prime}$ is in the space $C^{0}\left(\left[t_{0}, \infty\right) ; H_{\varepsilon}\right)$ and is the solution of the equation (4.1) on $\left(t_{0}, \infty\right)$. Our main objective here is to prove the estimates $(2.35),(2.36)$, and (2.37) of Theorem 2. The proof will be given in four steps. We will not use here the decomposition $u=v+w$. The results of Steps 1 and 2 are already contained in Lemma 3.0. However, we will reproduce the proof here because we require the precise estimates of $\int_{0}^{t}\left\|A_{\varepsilon} u(s)\right\|^{2} d s$ and $\int_{0}^{t}\left\|u^{\prime}(s)\right\|^{2} d s$ used to obtain (2.35), (2.36), and (2.37). As is usual, the formal estimates given here can be rigorously justified by using the Bubnov-Galerkin approximation method.

Step 1. First we derive an estimate for $\int_{\tau}^{t}\left\|A_{\varepsilon} u(s)\right\|^{2} d s$. Taking the scalar product of (2.5) with $A_{\varepsilon} u$, we obtain

$$
\frac{1}{2} \frac{d}{d t}\left\|A_{\varepsilon}^{1 / 2} u\right\|^{2}+\nu\left\|A_{\varepsilon} u\right\|^{2} \leq \frac{1}{\nu}\left\|\mathbb{P}_{\varepsilon} f\right\|_{\infty}^{2}+\frac{\nu}{4}\left\|A_{\varepsilon} u\right\|^{2}+\left|b_{\varepsilon}\left(u, u, A_{\varepsilon} u\right)\right|, \quad t \geq 0
$$

From the inequalities (8.8) and (2.18), we deduce that

$$
\left|b_{\varepsilon}\left(u^{1}, u^{2}, u^{3}\right)\right| \leq C_{9}\left\|A_{\varepsilon}^{1 / 2} u^{1}\right\|\left\|A_{\varepsilon}^{1 / 2} u^{2}\right\|^{1 / 2}\left\|A_{\varepsilon} u^{2}\right\|^{1 / 2}\left\|u^{3}\right\|
$$

for any $u^{1} \in D\left(A_{\varepsilon}^{1 / 2}\right), u^{2} \in D\left(A_{\varepsilon}\right), u^{3} \in H_{\varepsilon}$, where $C_{9}$ is a positive constant, independent on $\varepsilon$. This in turn implies that

$$
\left|b_{\varepsilon}\left(u, u, A_{\varepsilon} u\right)\right| \leq C_{9}\left\|A_{\varepsilon}^{1 / 2} u\right\|^{3 / 2}\left\|A_{\varepsilon} u\right\|^{3 / 2} \text {. }
$$


Using (4.4) and the Young inequality (3.4), we deduce from (4.2) that

$$
\frac{d}{d t}\left\|A_{\varepsilon}^{1 / 2} u\right\|^{2}+\nu\left\|A_{\varepsilon} u\right\|^{2} \leq \frac{2}{\nu}\left\|\mathbb{P}_{\varepsilon} f\right\|_{\infty}^{2}+\frac{27 C_{9}^{4}}{2 \nu^{3}}\left\|A_{\varepsilon}^{1 / 2} u\right\|^{6}, \quad t \geq 0
$$

By integrating (4.5) we infer that for $\max (0, t-1) \leq \tau \leq t$ one has

$\int_{\tau}^{t}\left\|A_{\varepsilon} u(s)\right\|^{2} d s \leq \frac{2}{\nu^{2}}(t-\tau)\left\|\mathbb{P}_{\varepsilon} f\right\|_{\infty}^{2}+D_{25}(t-\tau) \sup _{\tau \leq s \leq t}\left\|A_{\varepsilon}^{1 / 2} u(s)\right\|^{6}+\frac{1}{\nu}\left\|A_{\varepsilon}^{1 / 2} u(\tau)\right\|^{2}$,

where $D_{25}=27 C_{9}^{4} 2^{-1} \nu^{-4}$. Since the right-hand side of (4.6) is bounded for any $t>0$, it follows that the integrand on the left-hand side is finite almost everywhere. Therefore, there exists arbitrarily small $t_{0}>0$ such that $u\left(t_{0}\right) \in$ $D\left(A_{\varepsilon}\right)$.

Step 2. Next we derive an estimate of $\int_{\tau}^{t}\left\|u^{\prime}(s)\right\|^{2} d s$ for $0 \leq \tau \leq t$. First we observe that $(2.5)$ yields the identity

$$
\left\langle u^{\prime}, u^{\prime}\right\rangle=\left\langle\mathbb{P}_{\varepsilon} f, u^{\prime}\right\rangle-\left\langle\nu A_{\varepsilon} u, \mathbb{P}_{\varepsilon} f-\nu A_{\varepsilon} u-B_{\varepsilon}(u, u)\right\rangle-\left\langle B_{\varepsilon}(u, u), u^{\prime}\right\rangle,
$$

and consequently one finds that

$\left\|u^{\prime}\right\|^{2} \leq\left\|\mathbb{P}_{\varepsilon} f\right\|\left\|u^{\prime}\right\|+\nu\left\|\mathbb{P}_{\varepsilon} f\right\|\left\|A_{\varepsilon} u\right\|+\nu^{2}\left\|A_{\varepsilon} u\right\|^{2}+\nu\left|b_{\varepsilon}\left(u, u, A_{\varepsilon} u\right)\right|+\left|b_{\varepsilon}\left(u, u, u^{\prime}\right)\right|$.

By applying the Young inequality (3.4) several times and using (4.2) and (4.4), we obtain

$$
\left\|u^{\prime}\right\|^{2} \leq 3\left(\nu^{2}+1\right)\left\|A_{\varepsilon} u\right\|^{2}+\nu^{4} C_{9}^{4}\left\|A_{\varepsilon}^{1 / 2} u\right\|^{6}+3\left\|\mathbb{P}_{\varepsilon} f\right\|^{2}
$$

As a result one has

$$
\int_{\tau}^{t}\left\|u^{\prime}(s)\right\|^{2} d s \leq \int_{\tau}^{t}\left(3\left(\nu^{2}+1\right)\left\|A_{\varepsilon} u(s)\right\|^{2}+3\left\|\mathbb{P}_{\varepsilon} f\right\|_{\infty}^{2}+\nu^{4} C_{9}^{4}\left\|A_{\varepsilon}^{1 / 2} u(s)\right\|^{6}\right) d s
$$

for $t \geq \tau \geq 0$. The inequalities (4.6) and (4.8) imply that for $\max (0, t-1) \leq$ $\tau \leq t$ one has

$$
\int_{\tau}^{t}\left\|u^{\prime}(s)\right\|^{2} d s \leq D_{26}(t-\tau)\left\|\mathbb{P}_{\varepsilon} f\right\|_{\infty}^{2}+D_{27}(t-\tau) \sup _{\tau \leq s \leq t}\left\|A_{\varepsilon}^{1 / 2} u(s)\right\|^{6}+D_{28}\left\|A_{\varepsilon}^{1 / 2} u(\tau)\right\|^{2},
$$

where $D_{26}=3+6\left(\nu^{2}+1\right) \nu^{-2}, \quad D_{27}=3\left(\nu^{2}+1\right) D_{25}+\nu^{4} C_{9}^{4}$, and $D_{28}=$ $3\left(\nu^{2}+1\right) \nu^{-1}$.

Step 3. Next we shall derive an estimate of $\left\|u^{\prime}(t)\right\|^{2}$ for $t>0$. Let $t_{0}$ be fixed so that $0 \leq t_{0}<\frac{1}{2}$, and $t_{0}$ is close to 0 with $u\left(t_{0}\right) \in D\left(A_{\varepsilon}\right)$. By taking the scalar product of (4.1) with $u^{\prime}(t)$, we obtain

$$
\frac{1}{2} \frac{d}{d t}\left\|u^{\prime}\right\|^{2}+\nu\left\|A_{\varepsilon}^{1 / 2} u^{\prime}\right\|^{2} \leq \frac{1}{\nu}\left\|A_{\varepsilon}^{-1 / 2} \mathbb{P}_{\varepsilon} f^{\prime}\right\|_{\infty}^{2}+\frac{\nu}{4}\left\|A_{\varepsilon}^{1 / 2} u^{\prime}\right\|^{2}+\left|b_{\varepsilon}\left(u^{\prime}, u, u^{\prime}\right)\right|,
$$


for $t \geq t_{0}$. However,

$$
\begin{aligned}
\left|b_{\varepsilon}\left(u^{\prime}, u, u^{\prime}\right)\right| & =\left|\sum_{i, j=1}^{3} \int_{Q_{3}} u_{i}^{\prime} \varepsilon^{-\{i\}}\left(D_{i} u_{j}\right) u_{j}^{\prime} d x\right| \\
& \leq \sum_{i, j=1}^{3}\left(\int_{Q_{3}} \varepsilon^{-2\{i\}}\left(D_{i} u_{j}\right)^{2} d x\right)^{1 / 2}\left(\int_{Q_{3}}\left(u_{i}^{\prime}\right)^{2}\left(u_{j}^{\prime}\right)^{2} d x\right)^{1 / 2} \\
& \leq C\left\|A_{\varepsilon}^{1 / 2} u\right\|\left(\sum_{i, j=1}^{3}\left(\int_{Q_{3}}\left(u_{i}^{\prime}\right)^{2} d x\right)^{1 / 4}\left(\int_{Q_{3}}\left(u_{j}^{\prime}\right)^{6}\right)^{1 / 4}\right),
\end{aligned}
$$

which gives

$$
\left|b_{\varepsilon}\left(u^{\prime}, u, u^{\prime}\right)\right| \leq C_{10}\left\|A_{\varepsilon}^{1 / 2} u\right\|\left\|u^{\prime}\right\|^{1 / 2}\left\|A_{\varepsilon}^{1 / 2} u^{\prime}\right\|^{3 / 2}
$$

Once again by using the Young inequality (3.4), we infer from (4.10) and (4.11) that

(4.12) $\frac{d}{d t}\left\|u^{\prime}(t)\right\|^{2}+\nu\left\|A_{\varepsilon}^{1 / 2} u^{\prime}(t)\right\|^{2} \leq \frac{2}{\nu}\left\|A_{\varepsilon}^{-1 / 2} \mathbb{P}_{\varepsilon} f^{\prime}\right\|_{\infty}^{2}+D_{29}\left\|A_{\varepsilon}^{1 / 2} u(t)\right\|^{4}\left\|u^{\prime}(t)\right\|^{2}$

for $t \geq t_{0}$, where $D_{29}=27 C_{10}^{4} 2^{-1} \nu^{-3}$. Next we apply the uniform Gronwall inequality, as in (3.68), on (4.12) to obtain

$$
\begin{aligned}
\left\|u^{\prime}(t)\right\|^{2} \leq & \left(\frac{1}{t-\tau_{0}} \int_{\tau_{0}}^{t}\left\|u^{\prime}(s)\right\|^{2} d s+\int_{\tau_{0}}^{t} \frac{2}{\nu}\left\|A_{\varepsilon}^{-1 / 2} \mathbb{P}_{\varepsilon} f^{\prime}\right\|_{\infty}^{2}\right) \\
& \times \exp \left(\int_{\tau_{0}}^{t} D_{29}\left\|A_{\varepsilon}^{1 / 2} u(s)\right\|^{4} d s\right)
\end{aligned}
$$

where $\tau_{0}=\max \left(t_{0}, t-1\right)$. Therefore, by using (4.9) for $t_{0} \leq t \leq 1$ one has

$$
\begin{aligned}
\left\|u^{\prime}(t)\right\|^{2} \leq & \left(\frac{2}{\nu}\left\|A_{\varepsilon}^{-1 / 2} \mathbb{P}_{\varepsilon} f^{\prime}\right\|_{\infty}^{2}+D_{26}\left\|\mathbb{P}_{\varepsilon} f\right\|_{\infty}^{2}+D_{27} \sup _{t_{0} \leq s \leq t}\left\|A_{\varepsilon}^{1 / 2} u(s)\right\|^{6}\right. \\
& \left.+\frac{D_{28}}{t-t_{0}}\left\|A_{\varepsilon}^{1 / 2} u\left(t_{0}\right)\right\|^{2}\right) \\
& \times \exp \left(D_{29} \sup _{t_{0} \leq s \leq t}\left\|A_{\varepsilon}^{1 / 2} u(s)\right\|^{4}\right) .
\end{aligned}
$$

Likewise for $t \geq 1$ one has $\left(t-t_{0}\right) \geq \frac{1}{2}$ and (4.13), together with (4.9), implies that

$$
\begin{aligned}
\left\|u^{\prime}(t)\right\|^{2} \leq & \left(\frac{2}{\nu}\left\|A_{\varepsilon}^{-1 / 2} \mathbb{P}_{\varepsilon} f^{\prime}\right\|_{\infty}^{2}+D_{26}\left\|\mathbb{P}_{\varepsilon} f\right\|_{\infty}^{2}+D_{27} \sup _{t-1 \leq s \leq t}\left\|A_{\varepsilon}^{1 / 2} u(s)\right\|^{6}\right. \\
& \left.+2 D_{28} \sup _{t-1 \leq s \leq t}\left\|A_{\varepsilon}^{1 / 2} u(s)\right\|^{2}\right) \\
& \times \exp \left(D_{29} \sup _{t-1 \leq s \leq t}\left\|A_{\varepsilon}^{1 / 2} u(s)\right\|^{4}\right) .
\end{aligned}
$$


Let us now assume that $u_{0}$ belongs to $D\left(A_{\varepsilon}\right)$. Thanks to the Gronwall inequality, we deduce from (4.12) that one has

$$
\begin{aligned}
\left\|u^{\prime}(t)\right\|^{2} \leq & \left(\left\|u^{\prime}(0)\right\|^{2}+\frac{2}{\nu}\left\|A_{\varepsilon}^{-1 / 2} \mathbb{P}_{\varepsilon} f^{\prime}\right\|_{\infty}^{2}\right) \\
& \times \exp \left(D_{29} \sup _{0 \leq s \leq t}\left\|A_{\varepsilon}^{1 / 2} u(s)\right\|^{4}\right), \quad 0 \leq t \leq 1 .
\end{aligned}
$$

However, (4.7) implies that

$$
\left\|u^{\prime}(0)\right\|^{2} \leq 3\left(\nu^{2}+1\right)\left\|A_{\varepsilon} u_{0}\right\|^{2}+3\left\|\mathbb{P}_{\varepsilon} f\right\|_{\infty}^{2}+3 \nu^{4} C_{9}^{4}\left\|A_{\varepsilon}^{1 / 2} u_{0}\right\|^{2} .
$$

By combining the last two inequalities, we find that for $t \leq 1$ one has

$$
\begin{aligned}
\left\|u^{\prime}(t)\right\|^{2} \leq & \left(3\left(\nu^{2}+1\right)\left\|A_{\varepsilon} u_{0}\right\|^{2}+3 \nu^{4} C_{9}^{4}\left\|A_{\varepsilon}^{1 / 2} u_{0}\right\|^{2}\right. \\
& \left.+3\left\|\mathbb{P}_{\varepsilon} f\right\|_{\infty}^{2}+\frac{2}{\nu}\left\|A_{\varepsilon}^{-1 / 2} \mathbb{P}_{\varepsilon} f^{\prime}\right\|_{\infty}^{2}\right) \\
& \times \exp \left(D_{29} \sup _{0 \leq s \leq t}\left\|A_{\varepsilon}^{1 / 2} u(s)\right\|^{4}\right) .
\end{aligned}
$$

Step 4. In this last step we shall verify inequalities (2.35), (2.36), and (2.37). By taking the scalar product of (2.5) with $A_{\varepsilon} u$ we obtain

$$
\nu\left\|A_{\varepsilon} u\right\|^{2} \leq\left\|u^{\prime}\right\|\left\|A_{\varepsilon} u\right\|+\left\|\mathbb{P}_{\varepsilon} f\right\|\left\|A_{\varepsilon} u\right\|+\left|b_{\varepsilon}\left(u, u, A_{\varepsilon} u\right)\right| .
$$

By using the Young inequality (3.4) with (4.4) we find that

$$
\left\|A_{\varepsilon} u(t)\right\|^{2} \leq \frac{3}{\nu^{2}}\left\|u^{\prime}(t)\right\|^{2}+\frac{3}{\nu^{2}}\left\|\mathbb{P}_{\varepsilon} f\right\|_{\infty}^{2}+\frac{9^{3} C_{9}^{4}}{16 \nu^{4}}\left\|A_{\varepsilon}^{1 / 2} u(t)\right\|^{6}, \quad t \geq t_{0},
$$

and consequently $u(t) \in D\left(A_{\varepsilon}\right)$ for all $t \geq t_{0}$. Since $t_{0}$ can be chosen arbitrarily small, one has $u(t) \in D\left(A_{\varepsilon}\right)$ for all $t>0$. Inequalities (4.14) and (4.18) then imply that for $0<t_{0} \leq t \leq 1$ one has

$$
\begin{aligned}
\left\|A_{\varepsilon} u(t)\right\|^{2} \leq & \left(D_{30}\left\|A_{\varepsilon}^{-1 / 2} \mathbb{P}_{\varepsilon} f^{\prime}\right\|_{\infty}^{2}+D_{31}\left\|\mathbb{P}_{\varepsilon} f\right\|_{\infty}^{2}+D_{32} \sup _{0 \leq s \leq t}\left\|A_{\varepsilon}^{1 / 2} u(s)\right\|^{6}\right. \\
& \left.+\frac{D_{33}}{t-t_{0}}\left\|A_{\varepsilon}^{1 / 2} u\left(t_{0}\right)\right\|^{2}\right) \\
& \times \exp \left(D_{29} \sup _{0 \leq s \leq t}\left\|A_{\varepsilon}^{1 / 2} u(s)\right\|^{4}\right),
\end{aligned}
$$

where $D_{30}=6 \nu^{-3}, D_{31}=3 \nu^{-2}\left(D_{26}+1\right), D_{32}=3 \nu^{-2} D_{27}+9^{3} C_{9}^{4}(2 \nu)^{-4}$, and $D_{33}=3 \nu^{-2} D_{28}$. Since (4.19) is valid for any $t_{0}$ satisfying $0<t_{0}<t \leq 1$, we can replace $t_{0}$ with its limit value $t_{0}=0$ to obtain

$$
\begin{aligned}
\left\|A_{\varepsilon} u(t)\right\|^{2} \leq & \left(D_{30}\left\|A_{\varepsilon}^{-1 / 2} \mathbb{P}_{\varepsilon} f^{\prime}\right\|_{\infty}^{2}+D_{31}\left\|\mathbb{P}_{\varepsilon} f\right\|_{\infty}^{2}+D_{32} \sup _{0 \leq s \leq t}\left\|A_{\varepsilon}^{1 / 2} u(s)\right\|^{6}\right. \\
& \left.+D_{33} t^{-1}\left\|A_{\varepsilon}^{1 / 2} u_{0}\right\|^{2}\right) \\
& \times \exp \left(D_{29} \sup _{0 \leq s \leq t}\left\|A_{\varepsilon}^{1 / 2} u(s)\right\|^{4}\right)
\end{aligned}
$$


for $0<t \leq 1$. For $t \geq 1$ one obtains from (4.15) instead that $(4.21)$

$$
\begin{aligned}
\left\|A_{\varepsilon} u(t)\right\|^{2} \leq & \left(D_{30}\left\|A_{\varepsilon}^{-1 / 2} \mathbb{P}_{\varepsilon} f^{\prime}\right\|_{\infty}^{2}+D_{31}\left\|\mathbb{P}_{\varepsilon} f\right\|_{\infty}^{2}+D_{32} \sup _{t-1 \leq s \leq t}\left\|A_{\varepsilon}^{1 / 2} u(s)\right\|^{6}\right. \\
& \left.+2 D_{33} \sup _{t-1 \leq s \leq t}\left\|A_{\varepsilon}^{1 / 2} u(s)\right\|^{2}\right) \\
& \times \exp \left(D_{29} \sup _{t-1 \leq s \leq t}\left\|A_{\varepsilon}^{1 / 2} u(s)\right\|^{4}\right) .
\end{aligned}
$$

The quantities $K_{2}^{2}, K_{3}^{2}$, and $K_{4}^{2}$ appearing in (2.35) are now readily identified from (4.20), (4.21), and (2.31). In the case that $t \geq \widehat{T}_{1}+1$, where $\widehat{T}_{1}$ is given by Theorem 1 , we are able to use the bound (2.33) for $\left\|A_{\varepsilon}^{1 / 2} u\right\|^{2}$. As a result (4.21) implies that

$$
\left\|A_{\varepsilon} u(t)\right\|^{2} \leq L_{6}^{2} \stackrel{\text { def }}{=} \Gamma_{2}\left(L_{5}^{2}\right), \quad t \geq \widehat{T}_{1}+1
$$

where

$$
\Gamma_{2}(\rho)=\left(D_{30}\left\|A_{\varepsilon}^{-1 / 2} \mathbb{P}_{\varepsilon} f^{\prime}\right\|_{\infty}^{2}+D_{31}\left\|\mathbb{P}_{\varepsilon} f\right\|_{\infty}^{2}+2 D_{33} \rho+D_{32} \rho^{3}\right) \exp \left(D_{29} \rho^{2}\right) .
$$

Note that since $L_{5}^{2}$ does not depend on the initial condition $u_{0}$, it follows from (4.22) that $L_{6}^{2}$ is independent of $u_{0}$ as well. This completes the proof of (2.35) and (2.36).

Let us now assume that $u_{0}$ belongs to $D\left(A_{\varepsilon}\right)$. Then we deduce from (4.18) and (4.17) that for $t \leq 1$ one has

$$
\begin{aligned}
\left\|A_{\varepsilon} u(t)\right\|^{2} \leq & \left(D_{30}\left\|A_{\varepsilon}^{-1 / 2} \mathbb{P}_{\varepsilon} f^{\prime}\right\|_{\infty}^{2}+D_{34}\left\|\mathbb{P}_{\varepsilon} f\right\|_{\infty}^{2}+D_{35}\left\|A_{\varepsilon}^{1 / 2} u(t)\right\|^{6}\right. \\
& \left.+D_{36}\left\|A_{\varepsilon}^{1 / 2} u_{0}\right\|^{2}+D_{37}\left\|A_{\varepsilon} u_{0}\right\|^{2}\right) \\
& \times \exp \left(D_{29} \sup _{0 \leq s \leq t}\left\|A_{\varepsilon}^{1 / 2} u(s)\right\|^{4}\right)
\end{aligned}
$$

where $D_{34}=12 \nu^{-2}, \quad D_{35}=9^{3} C_{9}^{4}(2 \nu)^{-4}, \quad D_{36}=9 C_{9}^{4} \nu^{2}$, and $D_{37}=$ $9\left(\nu^{2}+1\right) \nu^{-2}$. The quantities $K_{5}^{2}, K_{6}^{2}$, and $K_{7}^{2}$ appearing in (2.37) are now readily identified in (4.23). This completes the proof of Theorem 2.

Remarks. 1. Depending on the choice of $\eta_{2}, \eta_{4}$, and $r$, one could have $L_{5}^{2} \rightarrow$ $\infty$ as $\varepsilon \rightarrow 0^{+}$. If this happens, then one finds that $L_{6}^{2} \rightarrow \infty$ as well. On the other hand, one can easily give conditions whereby both $L_{5}^{2}$ and $L_{6}^{2}$ are bounded for $0<\varepsilon \leq 1$. We will be treating the latter situation in detail in $\S 6$ wherein we prove the upper semicontinuity of the attractors at $\varepsilon=0$.

2. The decomposition $u=v+w$, as used in $\S 3$, together with the arguments used here, may lead to slight improvements in the estimates appearing in Theorem 2 .

3. The proof of the Theorem 3, which we give next, is similar to the argument in Babin and Vishik (1989, Theorem 2, $\S 6$, Chapter 1) for the 2DNS. 
Proof of Theorem 3. Let $f$ satisfy the hypothesis of Theorem 3 , and let $u_{0}$ be any point in $\mathscr{B}_{\varepsilon}^{0} \cup \mathscr{B}_{\varepsilon}{ }^{1}$. Let $\tau>0$ be fixed. Without loss of generality we will assume that $0<\tau \leq 1$. that

Assume for the moment that there is a compact set $\mathscr{K}^{0}(\tau)$ in $L^{2}\left(Q_{3}\right)$ such

$$
A_{\varepsilon} S_{\varepsilon}(f, t) u_{0} \in \mathscr{K}^{0}(\tau), \quad t \geq \tau, u_{0} \in \mathscr{B}_{\varepsilon}^{0} \cup \mathscr{B}_{\varepsilon}^{1},
$$

or equivalently

$$
S_{\varepsilon}(f, t)\left(\mathscr{B}_{\varepsilon}^{0} \cup \mathscr{B}_{\varepsilon}^{1}\right) \subset A_{\varepsilon}^{-1}\left(\mathscr{K}^{0}(\tau)\right), \quad t \geq \tau .
$$

The continuity of $A_{\varepsilon}^{-1}$ assures us that $\mathscr{K}(\tau) \stackrel{\text { def }}{=} A_{\varepsilon}^{-1}\left(\mathscr{K}^{0}(\tau)\right)$ is a compact set in $V_{\varepsilon}^{2}$. In order to prove (4.24) we will use the fact that if $\mathscr{K}_{1}, \mathscr{K}_{2}$ are compact sets in $L^{2}\left(Q_{3}\right)$, then $\mathscr{K}_{1}+\mathscr{K}_{2}$ is compact in $L^{2}\left(Q_{3}\right)$.

Since $H^{+}(f)$ is assumed to be compact, the sets $\operatorname{Ev}\left(H^{+}(f)\right)$ and $\mathscr{K}_{1} \stackrel{\text { def }}{=}$ $\operatorname{Ev}\left(\mathbb{P}_{\varepsilon} H^{+}(f)\right)$ are compact sets in $L^{2}\left(Q_{3}\right)$ that satisfy

$$
\mathbb{P}_{\varepsilon} g(t) \in \mathscr{K}_{1} \quad \text { for all } g \in H^{+}(f), t \geq 0
$$

see $\S 2.11$. Now the equation (2.5) can be rewritten as

$$
\nu A_{\varepsilon} u(t)=\mathbb{P}_{\varepsilon} f(t)-u^{\prime}(t)-B_{\varepsilon}(u(t), u(t)), \quad t>0 \text {. }
$$

Assume next that there are functions $L_{1}(\tau)$ and $L_{2}(\tau)$, defined for $\tau>0$, which depend only on $\nu, \lambda_{1}$, and $\eta_{i}, i=1,2,3,4$, such that

$$
\left\|A_{\varepsilon}^{1 / 2} u^{\prime}(t)\right\|^{2} \leq L_{1}(\tau), \quad t \geq \tau
$$

and

$$
\left\|A_{\varepsilon}^{1 / 2} B_{\varepsilon}(u(t), u(t))\right\|^{2} \leq L_{2}(\tau), \quad t \geq \tau .
$$

In this case there is a set $\mathscr{K}_{2}(\tau)$, which is bounded in $V_{\varepsilon}^{1}$ and compact in $H_{\varepsilon} \subset L^{2}\left(Q_{3}\right)$, such that

$$
-\left(u^{\prime}(t)+B_{\varepsilon}(u(t), u(t))\right) \in \mathscr{K}_{2}(\tau), \quad t \geq \tau .
$$

By combining (4.25), (4.26), and (4.29) one has

$$
\nu A_{\varepsilon} u(t) \in \mathscr{K}_{1}+\mathscr{K}_{2}(\tau), \quad t \geq \tau,
$$

which implies (4.24).

From Constantin and Foiaş (1988) we note that there is a constant $E_{3}$ such that $^{6}$

$$
\left|b_{\varepsilon}\left(u^{1}, u^{2}, A_{\varepsilon}^{1 / 2} u^{3}\right)\right| \leq E_{3}\left\|A_{\varepsilon} u^{1}\right\|\left\|A_{\varepsilon} u^{2}\right\|\left\|u^{3}\right\|, \quad u^{1}, u^{2} \in V_{\varepsilon}^{2}, u^{3} \in V_{\varepsilon}^{1} .
$$

Now (4.30) implies that $\left\|A_{\varepsilon}^{1 / 2} B_{\varepsilon}(u, u)\right\| \leq E_{3}\left\|A_{\varepsilon} u\right\|^{2}$, when $u \in V_{\varepsilon}^{2}$. Hence (2.35) implies that (4.28) holds. In order to prove (4.27), we note that from (2.30), (2.35), (2.41), (4.14), and (4.15) one has

$$
\left\|u^{\prime}(t)\right\|^{2}+\left\|A_{\varepsilon} u(t)\right\|^{2} \leq K_{8}^{2} \tau^{-1}, \quad t \geq \tau,
$$

\footnotetext{
${ }^{6}$ One can show that $E_{3}$ is independent of $\varepsilon$.
} 
where $K_{8}^{2}$ is a positive constant depending only on $\nu, \lambda_{1}$, and $\eta_{i}, i=$ $1,2,3,4$.

In order to prove (4.27) we derive once again some formal estimates, which can be justified rigorously by using the Bubnov-Galerkin approximation method. Let us now take the scalar product of (4.1) with $A_{\varepsilon} u^{\prime}$ for $t>0$. We obtain

$$
\begin{aligned}
& \frac{1}{2} \frac{d}{d t}\left\|A_{\varepsilon}^{1 / 2} u^{\prime}\right\|^{2}+\nu\left\|A_{\varepsilon} u^{\prime}\right\|^{2} \\
& \quad \leq \nu^{-1}\left\|\mathbb{P}_{\varepsilon} f^{\prime}\right\|_{\infty}^{2}+\frac{\nu}{4}\left\|A_{\varepsilon} u^{\prime}\right\|^{2}+\left|b_{\varepsilon}\left(u^{\prime}, u, A_{\varepsilon} u^{\prime}\right)\right|+\left|b_{\varepsilon}\left(u, u^{\prime}, A_{\varepsilon} u^{\prime}\right)\right| .
\end{aligned}
$$

However, using (4.3) and (4.30) one then obtains

$$
\frac{d}{d t}\left\|A_{\varepsilon}^{1 / 2} u^{\prime}(t)\right\|^{2}+\nu\left\|A_{\varepsilon} u^{\prime}(t)\right\|^{2} \leq 2 \nu^{-1}\left(\left\|\mathbb{P}_{\varepsilon} f^{\prime}\right\|_{\infty}^{2}+8 E_{4}^{2} K_{8}^{2} \tau^{-1}\left\|A_{\varepsilon}^{1 / 2} u^{\prime}(t)\right\|^{2}\right)
$$

for $t \geq \tau$, where $E_{4}^{2}=\left(C_{9}^{2}+E_{3}^{2}\right)\left(\lambda_{1}^{-1}+1\right)$. Now apply the uniform Gronwall inequality to (4.32) to obtain

$$
\begin{aligned}
\left\|A_{\varepsilon}^{1 / 2} u^{\prime}(t)\right\|^{2} \leq & \left(\frac{1}{t-\tau_{1}} \int_{\tau_{1}}^{t}\left\|A_{\varepsilon}^{1 / 2} u^{\prime}(s)\right\|^{2} d s+2 \nu^{-1}\left\|\mathbb{P}_{\varepsilon} f^{\prime}\right\|_{\infty}^{2}\left(t-\tau_{1}\right)\right) \\
& \times \exp \left(8 \nu^{-1} E_{4}^{2} K_{8}^{2} \tau^{-1}\left(t-\tau_{1}\right)\right)
\end{aligned}
$$

for $t \geq \tau$, where $\tau_{1}=\max (\tau, t-1)$.

It remains to estimate the term $\int_{\tau_{1}}^{t}\left\|A_{\varepsilon}^{1 / 2} u^{\prime}(s)\right\|^{2} d s$. Integrating the inequality (4.12) between $\tau_{1}$ and $t$ and using the estimates (2.30) and (4.31), we obtain

$$
\frac{1}{t-\tau_{1}} \int_{\tau_{1}}^{t}\left\|A_{\varepsilon}^{1 / 2} u^{\prime}(s)\right\|^{2} d s \leq \frac{2}{\nu^{2}}\left\|A_{\varepsilon}^{-1 / 2} \mathbb{P}_{\varepsilon} f^{\prime}\right\|_{\infty}^{2}+\frac{1}{\nu} K_{8}^{2} \tau^{-1}\left(1+D_{29} K_{1}^{4}\right)
$$

By combining (4.33) and (4.34) we deduce that (4.27) holds where

$$
\begin{aligned}
L_{1}(\tau)= & \left(\frac{2}{\nu^{2}}\left\|A_{\varepsilon}^{-1 / 2} \mathbb{P}_{\varepsilon} f^{\prime}\right\|_{\infty}^{2}+\frac{1}{\nu} K_{8}^{2} \tau^{-1}\left(1+D_{29} K_{1}^{4}\right)+\frac{2}{\nu}\left\|\mathbb{P}_{\varepsilon} f^{\prime}\right\|_{\infty}^{2}\right) \\
& \times \exp \left(8 \nu^{-1} E_{4}^{2} K_{8}^{2} \tau^{-1}\right),
\end{aligned}
$$

which completes the proof of Theorem 3 .

\section{THE REDUCED 3-DIMENSIONAL THEORY: THEOREM 6}

We return to the study of the reduced 3D Navier-Stokes evolutionary equation

$$
\bar{v}^{\prime}+\nu A_{\varepsilon} \bar{v}+B_{\varepsilon}(\bar{v}, \bar{v})=M \mathbb{P}_{\varepsilon} f,
$$

where $(I-M) \mathbb{P}_{\varepsilon} f=0$. In this case, $\{w=0\}$ is a positively invariant set for (2.5), the dilated Navier-Stokes evolutionary equation. Since $\bar{v}$ and $M \mathbb{P}_{\varepsilon} f$ do not depend on $x_{3}$, the terms in (5.1) do not depend on $\varepsilon$. Nevertheless the estimates derived in $\S \S 3$ and 4 are valid for (5.1); see, for example, Ladyzhenskaya (1969, 1972). 
We define $\alpha \stackrel{\text { def }}{=}\left\|A_{\varepsilon}^{1 / 2} \bar{v}_{0}\right\|$ and $\beta \stackrel{\text { def }}{=}\left\|M \mathbb{P}_{\varepsilon} f\right\|_{\infty}$. Let $L_{1}, L_{2}, \ldots$ denote functions of $\nu, \lambda_{1}$, and $\beta$ which are independent of $\alpha$ and $M_{1}, M_{2}, \ldots$ denote functions of $\nu, \lambda_{1}, \alpha$, and $\beta$. Let $D_{1}, D_{2}, \ldots$ be defined as in $\S 3$.

Instead of applying directly the results of Ladyzhenskaya $(1969,1972)$ we can use the estimates derived in $\S 3$ and take into account that $\bar{v}$ does not depend on $x_{3}$. Thus by defining $L_{1}=L_{1}(\beta)$ and $M_{1}=M_{1}(\alpha, \beta)$ as

$$
\left\{\begin{array}{l}
L_{1} \stackrel{\text { def }}{=} D_{18} \beta^{2} \exp \left(D_{19} \beta^{4}\right), \\
M_{1} \stackrel{\text { def }}{=} D_{18}\left(\alpha^{2} \exp \left(D_{19} \beta^{4}\right)+D_{19}\left(\alpha^{4}+2 \alpha^{2} \beta^{2}\right)\left(\alpha^{2}+\beta^{2}\right)\right) \exp \left(D_{19}\left(\alpha^{2}+\beta^{2}\right)^{2}\right),
\end{array}\right.
$$

we obtain the following result.

Theorem 6 (Part 1). Let $\bar{v}(t)$ be a solution of the reduced 3D Navier-Stokes evolutionary equation (5.1) with $\bar{v}_{0} \in M D\left(A_{\varepsilon}^{1 / 2}\right)$. Then there are functions

$$
M_{1}=M_{1}\left(\left\|A_{\varepsilon}^{1 / 2} \bar{v}_{0}\right\|^{2},\left\|M \mathbb{P}_{\varepsilon} f\right\|_{\infty}^{2}\right), \quad L_{1}=L_{1}\left(\left\|M \mathbb{P}_{\varepsilon} f\right\|_{\infty}^{2}\right)
$$

given by (5.2), such that

$$
\left\|A_{\varepsilon}^{1 / 2} \bar{v}(t)\right\|^{2} \leq M_{1} e^{-\nu \lambda_{1} t}+L_{1}, \quad t \geq 0 .
$$

Notice that, by the definition (3.84), one has

$$
L_{1} \leq \Gamma\left(\beta^{2}\right)
$$

Moreover, there exists a time $\tau_{1}>0$, such that one has $M_{1} e^{-\nu \lambda_{1} t} \leq L_{1}$ for $t \geq \tau_{1}$. Combining this with (5.3) and (5.4) we get

$$
\left\|A_{\varepsilon}^{1 / 2} \bar{v}(t)\right\|^{2} \leq 2 \Gamma\left(\beta^{2}\right), \quad t \geq \tau_{1} .
$$

If $\beta^{2} \leq \eta_{2}^{-2}$ and if $0<\varepsilon \leq \varepsilon_{0}$, we then have

$$
\left\|A_{\varepsilon}^{1 / 2} \bar{v}(t)\right\|^{2} \leq 2 \Gamma\left(\eta_{2}^{-2}\right) \leq\left(4 \eta_{1}^{-2}+k_{1}^{2} \eta_{3}^{-4}\right), \quad t \geq \tau_{1}
$$

that is, for $0<\varepsilon \leq \varepsilon_{0}$ and $t \geq \tau_{1}, \bar{v}(t)$ belongs to $\mathscr{B}_{\varepsilon}{ }^{1}$.

Let us denote by $S_{0}(g, t)$ the mapping generated on $M D\left(A_{\varepsilon}^{1 / 2}\right)$ by the strong solutions of equation (5.1), where $g=M \mathbb{P}_{\varepsilon} f$. Arguing as in $\S 4$, one has the following regularity result.

Theorem 6 (Part 2). If

$M \mathbb{P}_{\varepsilon} f \in C^{0}\left([0, \infty) ; M H_{\varepsilon}\right) \cap L^{\infty}\left((0, \infty) ; M H_{\varepsilon}\right) \cap W^{1, \infty}\left((0, \infty) ; M D\left(A_{\varepsilon}^{-1 / 2}\right)\right)$,

then there exist six positive functions $K_{i}^{*}=K_{i}^{*}\left(\left\|A_{\varepsilon}^{1 / 2} \bar{v}_{0}\right\|,\left\|M \mathbb{P}_{\varepsilon} f\right\|_{\infty}\right)$ such that

$$
\begin{cases}\left\|A_{\varepsilon} \bar{v}(t)\right\|^{2} \leq K_{1}^{* 2}+K_{2}^{* 2}\left\|A_{\varepsilon}^{-1 / 2} M \mathbb{P}_{\varepsilon} f^{\prime}\right\|_{\infty}^{2}+K_{3}^{* 2} t^{-1} & \text { for } 0<t \leq 1, \\ \left\|A_{\varepsilon} \bar{v}(t)\right\|^{2} \leq K_{1}^{* 2}+K_{2}^{* 2}\left\|A_{\varepsilon}^{-1 / 2} M \mathbb{P}_{\varepsilon} f^{\prime}\right\|_{\infty}^{2} & \text { for } t \geq 1 .\end{cases}
$$

Moreover, if $\bar{v}_{0}$ belongs to $M D\left(A_{\varepsilon}\right)$, then one has

$$
\left\|A_{\varepsilon} \bar{v}(t)\right\|^{2} \leq K_{4}^{* 2}+K_{5}^{* 2}\left\|A_{\varepsilon} \bar{v}_{0}\right\|^{2}+K_{6}^{* 2}\left\|A_{\varepsilon}^{-1 / 2} M \mathbb{P}_{\varepsilon} f^{\prime}\right\|_{\infty}^{2}, \quad 0 \leq t \leq 1 .
$$


Moreover, if $f$ belongs to $W\left(Q_{3}\right)$, then, for any $\tau>0, S_{0}(g, t) \bar{v}_{0}$ belongs to a compact set of $M V_{\varepsilon}^{2}$ for $t \geq \tau$, provided $\bar{v}_{0}$ belongs to a bounded set of $M V_{\varepsilon}^{1}$. Furthermore, if $f$ belongs to $W\left(Q_{3}\right) \cap W^{1, \infty}\left((0, \infty) ; L^{2}\left(Q_{3}\right)\right)$ and is chosen so that $H^{+}(f)$ is compact, then, for any bounded set $\mathscr{B}$ of $M V_{\varepsilon}^{1}$ and any $\tau>0$, $S_{0}(g, t) \mathscr{B}$ is included in a compact set $K_{0}(\tau, \mathscr{B})$ of $M V_{\varepsilon}^{2}$, for $t \geq \tau$.

If $H^{+}(f)$ is no longer compact, then, under the above hypotheses, we can prove that, for $t>0, S_{0}(g, t) \mathscr{B}$ is included in a compact set $\widetilde{K}_{0}(t, \mathscr{B})$ which may depend on $t$.

Assume now that $f \in W\left(Q_{3}\right) \cap W^{1, \infty}\left((0, \infty) ; L^{2}\left(Q_{3}\right)\right)$ is chosen so that $H^{+}(f)$ is compact. Due to Theorem 6 (Parts 1 and 2), $S_{0}(g, t)$ maps $M D\left(A_{\varepsilon}^{1 / 2}\right)$ into itself, is bounded dissipative in $M D\left(A_{\varepsilon}^{1 / 2}\right)$, and for $t \geq t_{1}>0$ is completely continuous in $M D\left(A_{\varepsilon}^{1 / 2}\right)$. Therefore, the skew-product semiflow $\pi_{0}(\bar{v}, g, t)=\left(S_{0}(g, t) \bar{v}_{0}, g_{t}\right)$ defined in $\S 2.11$ admits a global compact attractor $\mathfrak{A}_{0}(g)$ in $M D\left(A_{\varepsilon}^{1 / 2}\right) \times H^{+}(g)$; see, for example, Hale (1988, Theorem 2.4.7). Since, by Theorem 6 (Part 2), $S_{0}(g, t)$ is also bounded dissipative in $M D\left(A_{\varepsilon}\right)$ and for $t \geq t_{1}>0$ completely continuous in $M D\left(A_{\varepsilon}\right), \mathfrak{A}_{0}(g)$ is also the global compact attractor in $M D\left(A_{\varepsilon}\right) \times H^{+}(g)$. By the estimates (5.3) and (5.4), we have

$$
\begin{aligned}
\mathfrak{A}_{0}(g) & \subset\left\{u=\bar{v}+w:\left\|A_{\varepsilon}^{1 / 2} \bar{v}\right\|^{2} \leq L_{1} \leq \Gamma\left(\left\|M \mathbb{P}_{\varepsilon} f\right\|^{2}\right), w=0\right\} \times \omega(g) \\
& \subset \mathscr{B}_{\varepsilon}{ }^{1} \times \omega\left(M \mathbb{P}_{\varepsilon} f\right) .
\end{aligned}
$$

\section{Properties of attractors: Theorems 4 and 5}

We turn next to the proofs of Theorems 4 and 5 concerning the attractors for the Navier-Stokes equations. Let $\mathscr{B}_{\varepsilon}^{0}, \mathscr{B}_{\varepsilon}^{1}$, and $\mathscr{B}_{\varepsilon}^{2}$ be given by (2.38), (2.39), and (2.40). By Lemmas 3.1 and $3.2, \mathscr{B}_{\varepsilon}^{2}$ is well defined and is a bounded set in $V_{\varepsilon}^{1}$.

Proof of Theorem 4. Set $\mathscr{U}_{\varepsilon}^{2}=\mathscr{B}_{\varepsilon}^{2} \times H^{+}\left(\mathbb{P}_{\varepsilon} f\right)$. For $u_{0} \in V_{\varepsilon}^{1}$ and $f \in W\left(Q_{3}\right)$ with $\mathbb{P}_{\varepsilon} f \in W^{1, \infty}\left((0, \infty) ; L^{2}\left(Q_{3}\right)\right)$ we let

$$
\pi_{\varepsilon}\left(u_{0}, \mathbb{P}_{\varepsilon} f, \tau\right)=\left(S_{\varepsilon}\left(\mathbb{P}_{\varepsilon} f, \tau\right) u_{0},\left(\mathbb{P}_{\varepsilon} f\right)_{\tau}\right)
$$

denote the skew-product semiflow generated by the strong solutions of the dilated Navier-Stokes evolutionary equation (2.5); see $\S 2.11$. Let $\mathfrak{A}_{\varepsilon}=\omega\left(\mathscr{U}_{\varepsilon}^{2}\right)$ be the $\omega$-limit set of $\mathscr{U}_{\varepsilon}^{2}$ in $V_{\varepsilon}^{1} \times \mathbb{P}_{\varepsilon} W\left(Q_{3}\right)$, i.e.,

$$
\mathfrak{A}_{\varepsilon} \stackrel{\text { def }}{=} \bigcap_{\tau \geq 0} \text { Closure }_{V_{\varepsilon}^{1} \times \mathbb{P}_{\varepsilon} W\left(Q_{3}\right)}\left(\bigcup_{t \geq \tau} \pi_{\varepsilon}\left(\mathscr{U}_{\varepsilon}^{2}, t\right)\right) .
$$

It follows from (2.36) in Theorem 2 that for $\tau \geq \widehat{T}_{1}+1$ the set

$$
\bigcup_{t \geq \tau} S_{\varepsilon}\left(\mathbb{P}_{\varepsilon} f, t\right) \mathscr{B}_{\varepsilon}^{2}
$$


lies in a bounded set in $V_{\varepsilon}^{2}$ and, thus, a compact set in $V_{\varepsilon}^{1}$. Since $H^{+}\left(\mathbb{P}_{\varepsilon} f\right)$ is compact, it then follows that

$$
\text { Closure }_{V_{\varepsilon}^{1} \times \mathbb{P}_{\varepsilon} W\left(Q_{3}\right)}\left(\bigcup_{t \geq \tau} \pi_{\varepsilon}\left(\mathscr{U}_{\varepsilon}^{2}, t\right)\right)
$$

is a nonempty compact set in $V_{\varepsilon}^{1} \times H^{+}\left(\mathbb{P}_{\varepsilon} f\right)$ for each $\tau \geq \widehat{T}_{1}+1$. Consequently $\mathfrak{A}_{\varepsilon}$ is a nonempty compact invariant set in $V_{\varepsilon}^{1} \times H^{+}\left(\mathbb{P}_{\varepsilon} f\right)$. Since

$$
S_{\varepsilon}\left(\mathbb{P}_{\varepsilon} f, t\right) \mathscr{B}_{\varepsilon}^{2} \subset \mathscr{B}_{\varepsilon}^{2}, \quad t \geq 0,
$$

$\mathscr{U}_{\varepsilon}^{2}$ is a positively invariant neighborhood of $\mathfrak{A}_{\varepsilon}$. Therefore, $\mathfrak{A}_{\varepsilon}$ is a local attractor for the strong solutions of (2.5) in $V_{\varepsilon}^{1} \times H^{+}\left(\mathbb{P}_{\varepsilon} f\right)$, and the basin of attraction satisfies $\mathscr{B}_{\varepsilon}^{2} \times H^{+}\left(\mathbb{P}_{\varepsilon} f\right) \subset B\left(\mathfrak{A}_{\varepsilon}\right)$.

Remarks. 1. While the basin of attraction $B\left(\mathfrak{A}_{\varepsilon}\right)$ is a large set in $V_{\varepsilon}^{1} \times H^{+}\left(\mathbb{P}_{\varepsilon} f\right)$, we do not know whether $B\left(\mathfrak{A}_{\varepsilon}\right)=V_{\varepsilon}^{1} \times H^{+}\left(\mathbb{P}_{\varepsilon} f\right)$. As a result we do not know whether $\mathfrak{A}_{\varepsilon}$ is the global attractor of $\pi_{\varepsilon}$. The reason for this is that there may exist $u_{0} \in V_{\varepsilon}^{1}$ such that the solution $S_{\varepsilon}\left(\mathbb{P}_{\varepsilon} f, t\right) u_{0}$ is not globally regular. Because of this, the fact that Corollary 4.1 allows us to conclude that $\mathfrak{A}_{\varepsilon}$ is the global attractor in the space of Leray solutions and $B\left(\mathfrak{A}_{\varepsilon}\right)=H_{\varepsilon} \times H^{+}\left(\mathbb{P}_{\varepsilon} f\right)$ is all the more surprising.

2. The fact that the Leray solutions of (2.5) may not be unique is not a concern from the point of view of the dynamics. One can overcome this problem by using the Bebutov flow; see Sell (1973).

Proof of Corollary 4.1. For any Leray solution of (2.5) we use (3.35) to obtain $\frac{1}{t} \int_{0}^{t}\left\|A_{\varepsilon}^{1 / 2} u\right\|^{2} d s \leq \frac{\nu^{-1}}{t}\left\|u_{0}\right\|^{2}+2 \nu^{-2}\left(\left\|A_{\varepsilon}^{-1 / 2} M \mathbb{P}_{\varepsilon} f\right\|_{\infty}^{2}+\left\|A_{\varepsilon}^{-1 / 2}(I-M) \mathbb{P}_{\varepsilon} f\right\|_{\infty}^{2}\right)$ for all $t>0$. From (2.6), (2.22), (3.1), and (2.55) with $\lambda^{-1}>2 \nu^{-2} \max \left(\lambda_{1}^{-1}, C_{5}^{2}\right)$ we obtain

$$
\begin{aligned}
\frac{1}{t} \int_{0}^{t}\left\|A_{\varepsilon}^{1 / 2} u\right\|^{2} d s & \leq \frac{\nu^{-1}}{t}\left\|u_{0}\right\|^{2}+2 \nu^{-2}\left(\lambda_{1}^{-1}\left\|M \mathbb{P}_{\varepsilon} f\right\|_{\infty}^{2}+C_{5}^{2} \varepsilon^{2}\left\|(I-M) \mathbb{P}_{\varepsilon} f\right\|_{\infty}^{2}\right) \\
& \leq \frac{\nu^{-1}}{t}\left\|u_{0}\right\|^{2}+2 \nu^{-2}\left(\lambda_{1}^{-1} \eta_{2}^{-2}+C_{5}^{2} \varepsilon^{2+r} \eta_{4}^{-2}\right) \\
& \leq \frac{\nu^{-1}}{t}\left\|u_{0}\right\|^{2}+k \min \left(\eta_{1}^{-2}, \varepsilon^{p} \eta_{3}^{-2}\right)
\end{aligned}
$$

for all $t>0$ and $0<\varepsilon \leq \varepsilon_{10}(\lambda)$, where $0<k<1$. Therefore, for

$$
T=\frac{2 \nu^{-1}\left\|u_{0}\right\|^{2}}{(1-k) \min \left(\eta_{1}^{-2}, \varepsilon^{p} \eta_{3}^{-2}\right)}
$$

there is a $t_{0}, 0 \leq t_{0}<T$, such that

$$
\left\|A_{\varepsilon}^{1 / 2} u\left(t_{0}\right)\right\|^{2} \leq \min \left(\eta_{1}^{-2}, \varepsilon^{p} \eta_{3}^{-2}\right), \quad 0<\varepsilon \leq \varepsilon_{10} .
$$


For this $t_{0}$, it follows from (2.21) that

$$
\begin{aligned}
\left\|A_{\varepsilon}^{1 / 2} v\left(t_{0}\right)\right\|^{2} & \leq\left\|A_{\varepsilon}^{1 / 2} u\left(t_{0}\right)\right\|^{2} \leq \eta_{1}^{-2}, \\
\left\|A_{\varepsilon}^{1 / 2} w\left(t_{0}\right)\right\|^{2} & \leq\left\|A_{\varepsilon}^{1 / 2} u\left(t_{0}\right)\right\|^{2} \leq \varepsilon^{p} \eta_{3}^{-2} .
\end{aligned}
$$

Consequently for $0<\varepsilon \leq \min \left(\varepsilon_{0}, \varepsilon_{10}\right)$, where $\varepsilon_{0}$ is given by Theorem 1 , one has $u\left(t_{0}\right) \in \mathscr{B}_{\varepsilon}{ }^{1}$. Theorem 1 then implies that $u(t)$ is regular for all $t \geq t_{0}$. It follows that for the Leray solutions the basin of attraction $B\left(\mathfrak{A}_{\varepsilon}\right)$ is $H_{\varepsilon} \times H^{+}(f)$. Consequently $\mathfrak{A}_{\varepsilon}$ is the global attractor for the Leray solutions.

Remark. The concept of a weak attractor for the 3DNS was studied in Foias and Temam (1987). It follows from Corollary 4.1 that the weak attractor coincides with $\mathfrak{A}_{\varepsilon}$ for thin domains.

Proof of Corollary 4.2. The proof of the existence of a global attractor $\mathfrak{A}_{0}$ for the skew-product semiflow $\pi_{0}(\cdot, g, t)$ in $M D\left(A_{\varepsilon}^{1 / 2}\right) \times H^{+}(g)$, where $g=M \mathbb{P}_{\varepsilon} f$, has been given in $\S 5$. The proof of (2.56) follows from (5.6) and (5.4).

Assume now that $(I-M) \mathbb{P}_{\varepsilon} f=0$ and that $0<\varepsilon \leq \varepsilon_{0}$. Clearly, any solution $\bar{v}(t)$ of (2.24) with initial data $\bar{v}_{0} \in M D\left(A_{\varepsilon}^{1 / 2}\right)$ is a solution of the dilated Navier-Stokes evolutionary equation (2.5). As $\mathfrak{A}_{0}(g)$ is included in $\mathscr{B}_{\varepsilon}^{1} \times \omega\left(M \mathbb{P}_{\varepsilon} f\right) \subset B\left(\mathfrak{A}_{\varepsilon}\right)$ and is an invariant set for equation (2.5), it follows that $\mathfrak{A}_{0}(g) \subset \mathfrak{A}_{\varepsilon}$. Let us now show that $\mathfrak{A}_{\varepsilon} \subset \mathfrak{A}_{0}(g)$. Since $(I-M) \mathbb{P}_{\varepsilon} f=0$, inequality (3.53) takes on the form

$$
\left\|A_{\varepsilon}^{1 / 2} w(t)\right\|^{2} \leq k_{2}^{2} \varepsilon^{2+r} \eta_{4}^{-2} \exp \left(-\nu C_{5}^{-2} \varepsilon^{-2} t / 2\right), \quad t \geq 0
$$

provided $u_{0} \in \mathscr{B}_{\varepsilon}{ }^{1}$. This implies that the $u$-component of the $\omega$-limit set of $\mathscr{B}_{\varepsilon}{ }^{1} \times H^{+}\left(\mathbb{P}_{\varepsilon} f\right)$ belongs to the set of functions in $\mathscr{B}_{\varepsilon}{ }^{1}$ which are independent of the variable $x_{3}$, i.e., $\mathfrak{A}_{\varepsilon} \subset \mathfrak{A}_{0}(g)$.

Proof of Theorem 5 and Corollary 5.1. We begin with Theorem 5. Let us consider a sequence of positive numbers $\varepsilon_{n} \rightarrow 0$ as $n \rightarrow \infty$. Let $\mathscr{F}$ be any positively invariant compact subset of $W\left(Q_{3}\right) \cap W^{1, \infty}\left((0, \infty), L^{2}\left(Q_{3}\right)\right)$, and let $f_{n}$ be a sequence of functions $f_{n} \in \mathscr{F}$ that satisfies

$$
\lim _{n \rightarrow \infty}\left\|f_{n}-f_{0}\right\|_{\infty}=0
$$

where $f_{0} \in M \mathscr{F}$. Then each of the positive hulls $H^{+}\left(f_{n}\right)$ and $H^{+}\left(f_{0}\right)$ are compact sets in $\mathscr{F}$. We set $g_{n}=\mathbb{P}_{\varepsilon_{n}} f_{n}$ and $g_{0}=M \mathbb{P}_{\varepsilon_{n}} f_{0}$. According to the comments made in $\S 2.6, \mathbb{P}_{\varepsilon_{n}} f_{0}(t)$ belongs to $M H_{\varepsilon_{n}}$ for every $t$, and consequently

$$
g_{0}=M \mathbb{P}_{\varepsilon_{n}} f_{0}=\mathbb{P}_{\varepsilon_{n}} f_{0}=\left(\begin{array}{c}
\mathbb{P}_{2}\left(\begin{array}{l}
f_{01} \\
f_{02}
\end{array}\right) \\
f_{03}
\end{array}\right),
$$

where $f_{0}=\left(f_{01}, f_{02}, f_{03}\right)$. It follows from (6.1) and (6.2) and the fact that $\mathbb{P}_{\varepsilon_{n}}$ is a projection that

$$
\lim _{n \rightarrow \infty}\left\|M \mathbb{P}_{\varepsilon_{n}} f_{n}-g_{0}\right\|_{\infty}=0
$$


and

$$
\lim _{n \rightarrow \infty}\left\|(I-M) \mathbb{P}_{\varepsilon_{n}} f_{n}\right\|_{\infty}=0
$$

The last two conditions can be written as

$$
\lim _{n \rightarrow \infty}\left\|\mathbb{P}_{\varepsilon_{n}} f_{n}-g_{0}\right\|_{\infty}=0
$$

For every $n$, we consider the dilated Navier-Stokes evolutionary equation, i.e.,

$$
u^{\prime}+\nu A_{\varepsilon_{n}} u+B_{\varepsilon_{n}}(u, u)=g_{n} .
$$

Let $S_{\varepsilon_{n}}\left(g_{n}, t\right) u_{0 n}=u_{n}(t)=v_{n}(t)+w_{n}(t)$ denote the strong solution of the equation (6.5) with initial data $u_{0 n}$ in $V_{\varepsilon_{n}}^{1}$. We also consider the reduced 3D Navier-Stokes evolutionary equation

$$
\bar{v}^{\prime}+\nu A_{0} \bar{v}+B_{0}(\bar{v}, \bar{v})=g_{0},
$$

with initial data $\bar{v}(0)=\bar{v}_{0}$ in $V_{0}^{1}$. Let $S_{0}\left(g_{0}, t\right) \bar{v}_{0}$ denote the strong solution of (6.6) with initial condition $\bar{v}_{0} \in V_{0}^{1}$. It follows from (6.4) that there exist an integer $n_{1}$ and a positive constant $E_{0}$ such that

$$
\max \left(\left\|g_{0}\right\|_{\infty}^{2},\left\|M g_{n}\right\|_{\infty}^{2},\left\|g_{n}\right\|_{\infty}^{2}\right) \leq E_{0}, \quad n \geq n_{1} .
$$

According to Theorem 1 and Lemmas 3.1 and 3.2, every solution of (6.5) with initial data in the bounded set $\mathscr{B}_{\varepsilon_{n}}^{0}$ ultimately enters into the bounded set $\mathscr{B}_{\varepsilon_{n}}^{1}$ as well as the bounded set $\mathscr{B}_{\varepsilon_{n}}^{3}$ where

$$
\mathscr{B}_{\varepsilon_{n}}^{3} \stackrel{\text { def }}{=}\left\{u=v+w \in \mathscr{B}_{\varepsilon_{n}}^{1}:\left\|A_{\varepsilon_{n}}^{1 / 2} v\right\|^{2} \leq \Gamma\left(E_{0}\right),\left\|A_{\varepsilon_{n}}^{1 / 2} w\right\|^{2} \leq k_{2}^{2} \varepsilon_{n}^{2+r} \eta_{4}^{-2}\right\} .
$$

In particular, the (local) attractor $\mathfrak{A}_{\varepsilon_{n}}$ of (6.5), see Theorem 4 , is included in $\mathscr{B}_{\varepsilon_{n}}^{3} \times \omega\left(g_{n}\right)$, for $n \geq n_{1}$. Likewise, due to the property (5.6), the global attractor $\mathfrak{A}_{0}=\mathfrak{A}_{0}\left(g_{0}\right)$ of $(6.6)$ is included in the bounded set $\mathscr{B}_{0}^{3} \times \omega\left(g_{0}\right)$, where

$$
\mathscr{B}_{0}^{3}=\left\{u=v+w \in V_{\varepsilon_{n}}^{1}:\left\|A_{0}^{1 / 2} v\right\|^{2} \leq \Gamma\left(E_{0}\right), w=0\right\} .
$$

Note that, for every $n$ one has $M \mathscr{B}_{\varepsilon_{n}}^{3}=\mathscr{B}_{0}^{3}$. Now define $E_{1}=\Gamma\left(E_{0}\right)$.

For $\tau \in \mathbb{R}$, we let $f_{n, \tau}, g_{n, \tau}$, and $g_{0, \tau}$, denote the translate of $f_{n}, g_{n}$, and $g_{0}$; see $\S 2.11$ Then from (6.4) it follows that for every $\delta>0$ there is an integer $n_{2} \geq n_{1}$ such that

$$
\left\|g_{n, \tau}-g_{0, \tau}\right\|_{\infty} \leq \delta / 2, \quad n \geq n_{2}, \tau \geq 0 .
$$

Furthermore, there is a $T \geq 0$ such that

$$
\operatorname{dist}_{W\left(Q_{3}\right)}\left(g_{0, \tau}, \omega\left(g_{0}\right)\right)<\delta / 2, \quad \tau>T .
$$

It then follows that

$$
\operatorname{dist}_{W\left(Q_{3}\right)}\left(g_{n, \tau}, \omega\left(g_{0}\right)\right) \leq \delta, \quad n \geq n_{2}, \tau \geq T,
$$

which implies that the attractors $\omega\left(g_{n}\right)$ are upper semicontinuous as $n \rightarrow \infty$. 
In the remainder of the argument we shall use the weaker condition (6.3) in place of (6.1). As a result the argument now applies both to Theorem 5 and Corollary 5.1.

We claim that there exists an integer $n_{3} \geq n_{2}$ and two positive constants $k_{3}$ and $E_{2}$, with $E_{2} \geq \max \left(E_{0}, E_{1}\right)$, such that

$$
\left\|A_{\varepsilon}^{1 / 2} v_{n}(t)\right\|^{2} \leq E_{2}, \quad\left\|A_{\varepsilon}^{1 / 2} w_{n}(t)\right\|^{2} \leq k_{3}^{2} \varepsilon^{2+r} \eta_{4}^{-2},
$$

for $\varepsilon=\varepsilon_{n}, n \geq n_{3}$, and $t \geq 0$, provided $u_{0_{n}} \in \mathscr{B}_{\varepsilon}^{3}$. Furthermore, one has

$$
\left\|A_{0}^{1 / 2} \bar{v}(t)\right\|^{2} \leq E_{2}
$$

for $t \geq 0$, provided $\left(\bar{v}_{0}, 0\right) \in \mathscr{B}_{0}^{3}$. Indeed (6.8) and (6.9) are immediate consequences of (3.82), (3.84), and Theorem 6 (Part 1).

Now we want to compare the orbits of the dilated Navier-Stokes equation (6.5) with those of the reduced 3D Navier-Stokes equation (6.6) when $u_{0 n}$ belongs to $\mathscr{B}_{\varepsilon_{n}}^{3}$. To this end, we consider the equation satisfied by $z_{n}(t) \stackrel{\text { def }}{=}$ $v_{n}(t)-\bar{v}(t)$ where $z_{n}(0)=0$ (i.e., $\left.v_{n}(0)=\bar{v}(0)=v_{0 n}\right), w_{n}(0)=w_{0 n}$, and $u_{0 n}=v_{0 n}+w_{0 n}$ belongs to $\mathscr{B}_{\varepsilon_{n}}^{3}$. We have

$$
z_{n}^{\prime}+\nu A_{\varepsilon_{n}} z_{n}=\left(M g_{n}-g_{0}\right)-M\left(B_{\varepsilon_{n}}\left(u_{n}, u_{n}\right)-B_{\varepsilon_{n}}(\bar{v}, \bar{v})\right)
$$

and $M z_{n}=z_{n}$. Taking the inner product of (6.10) by $A_{\varepsilon_{n}} z_{n}$, we obtain

$$
\begin{aligned}
\frac{1}{2} \frac{d}{d t} \| & A_{\varepsilon_{n}}^{1 / 2} z_{n}\left\|^{2}+\nu\right\| A_{\varepsilon_{n}} z_{n} \|^{2} \\
\leq & \left\|M g_{n}-g_{0}\right\|_{\infty}\left\|A_{\varepsilon_{n}} z_{n}\right\| \\
& +\left|b_{\varepsilon_{n}}\left(v_{n}, v_{n}, A_{\varepsilon_{n}} z_{n}\right)-b_{\varepsilon_{n}}\left(\bar{v}, \bar{v}, A_{\varepsilon_{n}} z_{n}\right)\right|+\left|b_{\varepsilon_{n}}\left(w_{n}, w_{n}, A_{\varepsilon_{n}} z_{n}\right)\right|
\end{aligned}
$$

for $t \geq 0$. However, we can write

$(6.12)$

$\left|b_{\varepsilon_{n}}\left(v_{n}, v_{n}, A_{\varepsilon_{n}} z_{n}\right)-b_{\varepsilon_{n}}\left(\bar{v}, \bar{v}, A_{\varepsilon_{n}} z_{n}\right)\right|=\left|b_{\varepsilon_{n}}\left(z_{n}, \bar{v}, A_{\varepsilon_{n}} z_{n}\right)+b_{\varepsilon_{n}}\left(v_{n}, z_{n}, A_{\varepsilon_{n}} z_{n}\right)\right|$.

From inequality $(8.13)$ we obtain

$$
\left|b_{\varepsilon_{n}}\left(z_{n}, \bar{v}, A_{\varepsilon_{n}} z_{n}\right)\right| \leq c_{12}\left\|z_{n}\right\|_{L^{\infty}\left(Q_{3}\right)}\left\|A_{\varepsilon_{n}}^{1 / 2} \bar{v}\right\|\left\|A_{\varepsilon_{n}} z_{n}\right\| .
$$

Since $z_{n}$ does not depend on the variable $x_{3}$, we can apply the following Gagliardo-Nirenberg type inequality:

$$
\left\|z_{n}\right\|_{L^{\infty}\left(Q_{3}\right)} \leq c\left\|z_{n}\right\|_{H^{2}\left(Q_{3}\right)}^{1 / 2}\left\|z_{n}\right\|_{L^{2}\left(Q_{3}\right)}^{1 / 2}
$$

see Friedman (1964). The estimates (6.13), (6.14), and (2.18) imply that

$$
\left|b_{\varepsilon_{n}}\left(z_{n}, \bar{v}, A_{\varepsilon_{n}} z_{n}\right)\right| \leq C_{10}\left\|z_{n}\right\|^{1 / 2}\left\|A_{\varepsilon_{n}}^{1 / 2} \bar{v}\right\|\left\|A_{\varepsilon_{n}} z_{n}\right\|^{3 / 2}
$$


for some constant $C_{10}$. From (6.11), (6.12), (6.15), (3.2), and (3.3), we find that

$$
\begin{aligned}
\frac{1}{2} \frac{d}{d t} \| & A_{\varepsilon_{n}}^{1 / 2} z_{n}\left\|^{2}+\nu\right\| A_{\varepsilon_{n}} z_{n} \|^{2} \\
\leq & \left\|A_{\varepsilon_{n}} z_{n}\right\|\left(\left\|M g_{n}-g_{0}\right\|_{\infty}+C_{2} \varepsilon_{n}^{1 / 2}\left\|A_{\varepsilon_{n}}^{1 / 2} w\right\|^{3 / 2}\left\|A_{\varepsilon_{n}} w\right\|^{1 / 2}\right) \\
& +C_{1}\left\|v_{n}\right\|^{1 / 2}\left\|A_{\varepsilon_{n}}^{1 / 2} v_{n}\right\|^{1 / 2}\left\|A_{\varepsilon_{n}}^{1 / 2} z_{n}\right\|^{1 / 2}\left\|A_{\varepsilon_{n}} z_{n}\right\|^{3 / 2} \\
& +C_{10}\left\|z_{n}\right\|^{1 / 2}\left\|A_{\varepsilon_{n}}^{1 / 2} \bar{v}\right\|\left\|A_{\varepsilon_{n}} z_{n}\right\|^{3 / 2}
\end{aligned}
$$

for $t \geq 0$. Using the Young inequality we derive from (6.16) that one has

$$
\begin{aligned}
\frac{d}{d t}\left\|A_{\varepsilon_{n}}^{1 / 2} z_{n}\right\|^{2}+\nu\left\|A_{\varepsilon_{n}} z_{n}\right\|^{2} & \\
\leq & \frac{4}{\nu}\left\|M g_{n}-g_{0}\right\|_{\infty}^{2}+\frac{4}{\nu} C_{2}^{2} \varepsilon_{n}\left\|A_{\varepsilon_{n}}^{1 / 2} w\right\|^{3}\left\|A_{\varepsilon_{n}} w\right\| \\
& +\frac{108}{\nu^{3}}\left(C_{1}^{4}\left\|v_{n}\right\|^{2}\left\|A_{\varepsilon_{n}}^{1 / 2} v_{n}\right\|^{2}\left\|A_{\varepsilon_{n}}^{1 / 2} z_{n}\right\|^{2}+C_{10}^{4}\left\|z_{n}\right\|^{2}\left\|A_{\varepsilon_{n}}^{1 / 2} \bar{v}\right\|^{4}\right)
\end{aligned}
$$

for $t \geq 0$, or, by (6.8) and (6.9),

$$
\begin{aligned}
\frac{d}{d t}\left\|A_{\varepsilon_{n}}^{1 / 2} z_{n}\right\|^{2}+\nu\left\|A_{\varepsilon_{n}} z_{n}\right\|^{2} \leq & \frac{4}{\nu}\left\|M g_{n}-g_{0}\right\|_{\infty}^{2}+\frac{4}{\nu} C_{2}^{2} \varepsilon_{n}\left\|A_{\varepsilon_{n}}^{1 / 2} w\right\|^{3}\left\|A_{\varepsilon_{n}} w\right\| \\
& +D_{24} E_{2}^{2}\left\|A_{\varepsilon_{n}}^{1 / 2} z_{n}\right\|^{2}
\end{aligned}
$$

where $D_{24}=108 \lambda_{1}^{-1} \nu^{-3}\left(C_{1}^{4}+C_{10}^{4}\right)$.

Integrating the inequality (6.17) from 0 to $t$ and using a Gronwall inequality, we deduce that

$$
\begin{aligned}
\left\|A_{\varepsilon_{n}}^{1 / 2} z_{n}(t)\right\|^{2} \leq & \frac{4}{\nu}\left(t\left\|M g_{n}-g_{0}\right\|_{\infty}^{2}+C_{2}^{2} \varepsilon_{n} \int_{0}^{t}\left\|A_{\varepsilon_{n}}^{1 / 2} w(s)\right\|^{3}\left\|A_{\varepsilon_{n}} w(s)\right\| d s\right) \\
& \times \exp \left(D_{24} E_{2}^{2} t\right)
\end{aligned}
$$

for $t \geq 0$. Arguing as in the proof of (3.54), we see that there exists a positive constant $D_{25}$ such that

$$
\frac{4}{\nu} C_{2}^{2} \varepsilon_{n} \int_{0}^{t}\left\|A_{\varepsilon_{n}}^{1 / 2} w(s)\right\|^{3}\left\|A_{\varepsilon_{n}} w(s)\right\| d s \leq D_{25}(1+t) \varepsilon_{n}^{4+2 r} \eta_{4}^{-4}, \quad t \geq 0 .
$$

Finally, we obtain

$$
\left\|A_{\varepsilon_{n}}^{1 / 2} z_{n}(t)\right\|^{2} \leq\left(\frac{4}{\nu} t\left\|M g_{n}-g_{0}\right\|^{2}+D_{25}(1+t) \varepsilon_{n}^{4+2 r} \eta_{4}^{-4}\right) \exp \left(D_{24} E_{2}^{2} t\right) .
$$

Thanks to hypothesis (2.59) and condition (6.3), we infer, from (6.18) that, for any positive numbers $\delta$ and $T$, there exists an integer $n_{4}, n_{4} \geq n_{3} \geq 0$, such that

$$
\left\|A_{\varepsilon_{n}}^{1 / 2} z_{n}(T)\right\|^{2} \leq \delta / 3, \quad n \geq n_{4} .
$$

Let $\delta$ be a positive number. Since $\mathfrak{A}_{0}$ is the global attractor of (6.6), there exists a positive time $\tau_{0} \equiv \tau_{0}(\delta)$ such that

$$
\pi_{0}\left(\mathscr{B}_{0}^{3}, H^{+}\left(g_{0}\right), t\right) \subset \mathscr{N}_{V_{0}^{1} \times W\left(Q_{3}\right)}\left(\mathfrak{A}_{0}, \delta / 3\right), \quad t \geq \tau_{0},
$$


where $\mathscr{N}_{V_{0}^{1} \times W\left(Q_{3}\right)}\left(\mathfrak{A}_{0}, \alpha\right)$ denotes the $\alpha$-neighborhood of $\mathfrak{A}_{0}$ in $V_{0}^{1} \times W\left(Q_{3}\right)$. Using the properties (6.8) and (6.19), as well as the hypothesis (2.59), we see that exists an integer $n_{0}, n_{0} \geq n_{4}$, such that

$$
\left\|A_{\varepsilon_{n}}^{1 / 2}\left(v_{n}\left(\tau_{0}\right)-\bar{v}\left(\tau_{0}\right)\right)\right\|^{2}+\left\|A_{\varepsilon_{n}}^{1 / 2} w_{n}\left(\tau_{0}\right)\right\|^{2} \leq 2 \delta / 3, \quad n \geq n_{0},
$$

where $u_{n}(t)=v_{n}(t)+w_{n}(t)=S_{\varepsilon_{n}}\left(g_{n}, t\right) u_{0 n}, \bar{v}(t)=S_{0}\left(g_{0}, t\right) M u_{0 n}$, and $u_{0 n} \in$ $\mathscr{B}_{\varepsilon_{n}}^{3}$. From (6.7), (6.20), and (6.21), we infer that

$$
\pi_{\varepsilon_{n}}\left(\mathscr{B}_{\varepsilon_{n}}^{3}, H^{+}\left(g_{n}\right), \tau_{0}\right) \subset \mathscr{N}_{V_{\varepsilon_{n}}^{1} \times W\left(Q_{3}\right)}\left(\mathfrak{A}_{0}, \delta\right), \quad n \geq n_{0},
$$

and, in particular,

$$
\pi_{\varepsilon_{n}}\left(\mathfrak{A}_{\varepsilon_{n}}, \tau_{0}\right) \subset \mathcal{N}_{V_{\varepsilon_{n}}^{1} \times W\left(Q_{3}\right)}\left(\mathfrak{A}_{0}, \delta\right), \quad n \geq n_{0} .
$$

Due to the invariance property of the attractors $\mathfrak{A}_{\varepsilon_{n}}$, we at once deduce the upper semicontinuity result (2.61) from (6.22). This completes the proof of Theorem 5 and Corollary 5.1 .

Proof of Corollary 5.2. We shall only give a sketch of the proof of Corollary 5.2. We keep here the notation of the proof of Theorem 5.

According to Theorems 2 and 6 (Part 2), every solution of (6.5), for $n \geq n_{1}$ (resp. of (6.6)), with initial data in the bounded set $\mathscr{B}_{\varepsilon_{n}}^{0}$ (resp. in any bounded set of $V_{0}^{1}$ ) ultimately enters into the bounded set $\mathscr{B}_{\varepsilon_{n}}^{4}{ }^{n}$ (resp. $\mathscr{B}_{0}^{4}$ ) where

$$
\mathscr{B}_{\varepsilon_{n}}^{4} \stackrel{\text { def }}{=}\left\{u \in V_{\varepsilon_{n}}^{2}:\left\|A_{\varepsilon_{n}} u\right\|^{2} \leq E_{4}\right\}
$$

(resp. $\mathscr{B}_{0}^{4}=\left\{v \in V_{0}^{2}:\left\|A_{0} v\right\|^{2} \leq E_{4}\right\}$ ) where $E_{4}$ is a positive constant independent of $n$. Note that, for every $n, M \mathscr{B}_{\varepsilon_{n}}^{4}=\mathscr{B}_{0}^{4}$. In particular, the (local) attractor $\mathfrak{A}_{\varepsilon_{n}}$ of (6.5) (resp. the global attractor $\mathfrak{A}_{0}$ of (6.6)) is included in the bounded set $\mathscr{B}_{\varepsilon_{n}}^{4} \times \omega\left(g_{n}\right)$ (resp. $\left.\mathscr{B}_{0}^{4} \times \omega\left(g_{0}\right)\right)$. Furthermore, due to Theorems 2 and 6 (Part 2), there exist an integer $n_{5}, n_{5} \geq n_{4}$, and a positive constant $E_{5}$, with $E_{5} \geq \max \left(E_{4}, E_{2}\right)$ such that, for $t \geq 0$,

$$
\left\|A_{\varepsilon_{n}} u_{n}(t)\right\|^{2} \leq E_{5} \quad \text { for } n \geq n_{5}, u_{0 n} \in \mathscr{B}_{\varepsilon_{n}}^{3} \cap \mathscr{B}_{\varepsilon_{n}}^{4},
$$

and

$$
\left\|A_{0} \bar{v}(t)\right\|^{2} \leq E_{5} \quad \text { for } \bar{v}_{0} \in \mathscr{B}_{0}^{3} \cap \mathscr{B}_{0}^{4} .
$$

Let $\delta$ be a positive number. Since $\mathfrak{A}_{0}$ is the global attractor of $(6.6)$ in $V_{0}^{2} \times$ $W\left(Q_{3}\right)$, there exists a positive time $\tau_{1} \equiv \tau_{1}(\delta)$, with $\tau_{1}>1$ for instance, such that

$$
\pi_{0}\left(\mathscr{B}_{0}^{3} \cap \mathscr{B}_{0}^{4}, H^{+}\left(g_{0}\right), t\right) \subset \mathscr{N}_{V_{0}^{2} \times W\left(Q_{3}\right)}\left(\mathfrak{A}_{0}, \delta / 3\right), \quad t \geq \tau_{1} .
$$

As in the proof of Theorem 5, due to the properties (6.7) and (6.23), the upper semicontinuity result $(2.63)$ is valid if we show that there exists an integer $n_{6}$, $n_{6} \geq n_{5}$, such that, for $n \geq n_{6}$, one has

$$
\left\|A_{\varepsilon_{n}}\left(u_{n}\left(\tau_{1}\right)-\bar{v}\left(\tau_{1}\right)\right)\right\|^{2} \leq 2 \delta / 3
$$


where $u_{n}(t)=S_{\varepsilon_{n}}\left(g_{n}, t\right) u_{0 n}, \bar{v}(t)=S_{0}\left(g_{0}, t\right) \bar{v}_{0}$, and $u_{0 n} \in \mathscr{B}_{\varepsilon_{n}}^{3} \cap \mathscr{B}_{\varepsilon_{n}}^{4}$.

Note that $z_{n} \stackrel{\text { def }}{=} u_{n}-\bar{v}$ is the solution of the equation

$$
z_{n}^{\prime}+\nu A_{\varepsilon_{n}} z_{n}=\left(g_{n}-g_{0}\right)-\left(B_{\varepsilon_{n}}\left(u_{n}, u_{n}\right)-B_{\varepsilon_{n}}(\bar{v}, \bar{v})\right)
$$

with initial condition $z_{n}(0)=u_{0 n}-M u_{0 n}=w_{0 n}$. The proof of the estimate (6.24) follows the lines of the proof of the estimates (2.35), (2.36) of Theorem 2 (see $\S 4$, Steps 1 to 4 ). As the proof of (6.24) is rather long and completely similar to the proof of Theorem 2, we omit the details. Let us just point out that, as in $\S 4$, we use the auxiliary equation

$\frac{d}{d t} z_{n}^{\prime}+\nu A_{\varepsilon_{n}} z_{n}^{\prime}=\left(g_{n}^{\prime}-g_{0}^{\prime}\right)-\left(B_{\varepsilon_{n}}\left(u_{n}^{\prime}, u_{n}\right)+B_{\varepsilon_{n}}\left(u_{n}, u_{n}^{\prime}\right)-B_{\varepsilon_{n}}\left(\bar{v}^{\prime}, \bar{v}\right)-B_{\varepsilon_{n}}\left(\bar{v}, \bar{v}^{\prime}\right)\right)$,

with initial condition

$$
z_{n}^{\prime}(0)=\left(g_{n}-g_{0}\right)(0)-\left(B_{\varepsilon_{n}}\left(u_{0 n}, u_{0 n}\right)-B_{\varepsilon_{n}}\left(v_{0 n}, v_{0 n}\right)\right)-\nu A_{\varepsilon_{n}}\left(u_{0 n}-v_{0 n}\right) \text {. }
$$

\section{REMARKS ON OTHER BOUNDARY CONDITIONS: THEOREM 7}

In this section, we assume that $\Omega_{\varepsilon}=Q_{2} \times(0, \varepsilon)$, where $Q_{2}$ is a bounded domain in $\mathbb{R}^{2}$ with a boundary of class $C^{s}, s \geq 2$. The smoothness hypothesis $s \geq 2$ is made to avoid any problem of regularity of the solutions of the corresponding stationary Stokes equation. As in $\S 2$, we set $Q_{3}=Q_{2} \times(0,1)$ and use the change of variables $\left(y_{1}, y_{2}, y_{3}\right) \mapsto\left(x_{1}, x_{2}, x_{3}\right)$, where $x_{i}=y_{i}$, $i=1,2$, and $x_{3}=\varepsilon^{-1} y_{3}$. This change of variables sends $\Omega_{\varepsilon}$ onto $Q_{3}$.

7.1. Mixed periodic-Dirichlet boundary conditions. We are interested here in solutions of the Navier-Stokes evolutionary equation (2.5) that satisfy periodic boundary conditions on $\Gamma_{1}=Q_{2} \times\{0\} \cup Q_{2} \times\{\varepsilon\}$ and Dirichlet boundary conditions on $\Gamma_{2}=\partial Q_{2} \times(0, \varepsilon)$. As before we use the operator $J_{\varepsilon}$ of $\S 2.1$. Let $H_{\varepsilon}$ (respectively $V_{\varepsilon}^{1}$ ) denote the closure in $L^{2}\left(Q_{3}\right)$ (respectively $H^{1}\left(Q_{3}\right)$ ) of those smooth functions $u$ that satisfy periodic boundary conditions on $\Gamma_{1}$, Dirichlet boundary conditions on $\Gamma_{2}$, and $\nabla_{\varepsilon} \cdot u=0$ in $Q_{3}$. We denote by $\mathbb{P}_{\varepsilon}$ the orthogonal projection of $L^{2}\left(Q_{3}\right)$ onto $H_{\varepsilon}$. By applying $\mathbb{P}_{\varepsilon}$ to (2.4), we obtain (as in $\S 2.2$ ) the nonlinear evolutionary equation (2.5) on $H_{\varepsilon}$, where $u \in H_{\varepsilon}, A_{\varepsilon} u=-\mathbb{P}_{\varepsilon} \Delta_{\varepsilon} u$. We set $V_{\varepsilon}^{2}=D\left(A_{\varepsilon}\right)$. Using regularity results (see Dauge (1984) and the references therein), one can show that $V_{\varepsilon}^{2}=V_{\varepsilon}^{1} \cap H^{2}\left(Q_{3}\right)$. One also has $V_{\varepsilon}^{1}=D\left(A_{\varepsilon}^{1 / 2}\right)$. Using the classical Poincaré inequality, one easily shows that the inequalities (2.17) still hold. Likewise, thanks to the estimates (2.17) and to regularity results in Dauge (1984), one can prove the inequalities (2.18). Like in $\S 2.4$, we introduce the projection $M$. All the properties given in $\S 2.4$ are still true. In particular, if $u \in D\left(A_{\varepsilon}\right)$, we have

$$
(I-M) A_{\varepsilon} u=A_{\varepsilon}(I-M) u .
$$

The crucial estimate (2.22) still holds (see Hale and Raugel (1989)). The above property (7.1) allows us to write the equation (2.5) as the system (2.23) of two equations in $v=M u$ and $w=(I-M) u$. 
As in $\S 2.6$, we obtain a reduced 3D Navier-Stokes evolutionary equation which is given by (2.24). The reduced 3D Navier-Stokes evolutionary equation incorporates the 2DNS equation on $Q_{2}$ with homogeneous Dirichlet boundary conditions. In order to see this, we let $L^{2}\left(Q_{2}, \mathbb{R}^{2}\right)$ denote the $L^{2}$-space of 2 dimensional vector fields $m=\left(m_{1}, m_{2}\right)$ which depend on $\left(x_{1}, x_{2}\right) \in Q_{2}$ and let $H\left(Q_{2}\right)$ denote the closure in $L^{2}\left(Q_{2}, \mathbb{R}^{2}\right)$ of the smooth functions $u$ that satisfy $D_{1} m_{1}+D_{2} m_{2}=0$ on $Q_{2}$. Finally we let $\mathbb{P}_{2}$ denote the orthogonal projection of $L^{2}\left(Q_{2}, \mathbb{R}^{2}\right)$ onto the space $H\left(Q_{2}\right)$. Then $\mathbb{P}_{\varepsilon}$ and $\mathbb{P}_{2}$ satisfy the relations described in $\S 2.6$. Furthermore, $\bar{v}$ is a solution of the reduced 3D Navier-Stokes evolutionary equation (2.24) if and only if $m=\left(\bar{v}_{1}, \bar{v}_{2}\right)$ is a solution of the 2D Navier-Stokes evolutionary equation

$$
\frac{d}{d t} m-\nu \mathbb{P}_{2}\left(D_{1}^{2}+D_{2}^{2}\right) m+\mathbb{P}_{2}(m \cdot \nabla) m=\left(g_{1}, g_{2}\right)
$$

and $\bar{v}_{3}$ is a solution of the linear equation

$$
\frac{d}{d t} \bar{v}_{3}-\nu\left(D_{1}^{2}+D_{2}^{2}\right) \bar{v}_{3}+\left(\bar{v}_{1} D_{1}+\bar{v}_{2} D_{2}\right) \bar{v}_{3}=g_{3}
$$

where $g=\left(g_{1}, g_{2}, g_{3}\right)=M \mathbb{P}_{\varepsilon} f$. With the changes made above in the definitions of the spaces $H_{\varepsilon}, V_{\varepsilon}^{1}, V_{\varepsilon}^{2}$ and the operators $\mathbb{P}_{\varepsilon}$ and $\mathbb{P}_{2}$, all the results given in $\S \S 2-6$ (see also $\S 8$ ) are still true in the case where we have periodic boundary conditions on $\Gamma_{0} \cup \Gamma_{1}$ and homogeneous Dirichlet boundary conditions on $\Gamma_{2}$. Moreover, the proofs given in $\S \S 3-6$ are exactly the same.

7.2. Homogeneous Dirichlet boundary conditions. This case is quite different from the cases previously studied. Here we consider the Navier-Stokes equations (2.1) on $\Omega_{\varepsilon}$ (resp. (2.4) on $Q_{3}$ ) with homogeneous Dirichlet boundary conditions on $\partial \Omega_{\varepsilon}$ (resp. on $\left.\partial Q_{3}\right)$. Here we introduce the spaces

$$
H_{\varepsilon}=\left\{u \in L^{2}\left(Q_{3}\right): \nabla_{\varepsilon} \cdot u=0,\left.u \cdot n\right|_{\partial Q_{3}}=0\right\}
$$

and

$$
V_{\varepsilon}^{1}=\left\{u \in H_{0}^{1}\left(Q_{3}\right): \nabla_{\varepsilon} \cdot u=0\right\},
$$

and we denote by $\mathbb{P}_{\varepsilon}$ the orthogonal projection of $L^{2}\left(Q_{3}\right)$ onto $H_{\varepsilon}$. By applying $\mathbb{P}_{\varepsilon}$ to $(2.4)$, we obtain (as in $\S 2.2$ ) the dilated Navier-Stokes evolutionary equation (2.5) where $u=\mathbb{P}_{\varepsilon} u \in H_{\varepsilon}, A_{\varepsilon} u=-\mathbb{P}_{\varepsilon} \Delta_{\varepsilon} u$ (with homogenous Dirichlet boundary conditions). One has $V_{\varepsilon}^{1}=D\left(A_{\varepsilon}^{1 / 2}\right)$ and we set $V_{\varepsilon}^{2}=D\left(A_{\varepsilon}\right)$. Using the regularity results given in Dauge $(1984,1989)$, one obtains that $V_{\varepsilon}^{2}=V_{\varepsilon}^{1} \cap H^{2}\left(Q_{3}\right)$. Using the classical Poincaré inequality, one shows at once that the estimates (2.17) still hold. Arguing as in Hale and Raugel (1992a, Corollary 2.8 ) one shows that

$$
\left\|A_{\varepsilon}^{i} u\right\| \leq C_{11} \varepsilon\left\|A_{\varepsilon}^{i+1 / 2} u\right\| \text { for } i=0,1 \text {, }
$$

where $C_{11}$ is a positive constant that does not depend on $\varepsilon$. Using the inequality (7.2) several times and the regularity results of Dauge (1984, 1989), one proves that

$$
C_{6}\left(\|u\|_{H^{2}(Q)}+\varepsilon^{-1}\left\|D_{3} u\right\|+\varepsilon^{-1}\left\|D_{1} D_{3} u\right\|+\varepsilon^{-1}\left\|D_{2} D_{3} u\right\|+\varepsilon^{-2}\left\|D_{3}^{2} u\right\|\right) \leq \varepsilon^{-1}\left\|A_{\varepsilon} u\right\|
$$


and

$$
\left\|A_{\varepsilon} u\right\| \leq C_{7}\left(\|u\|_{H^{2}\left(Q_{3}\right)}+\varepsilon^{-1}\left\|D_{3} u\right\|_{H^{1}\left(Q_{3}\right)}+\varepsilon^{-2}\left\|D_{3}^{2} u\right\|\right) .
$$

The inequalities (7.3) and (8.20) imply that

$$
\left|b_{\varepsilon}\left(u^{1}, u^{2}, u^{3}\right)\right| \leq C_{12}\left\|A_{\varepsilon}^{1 / 2} u^{1}\right\|\left\|A_{\varepsilon}^{1 / 2} u^{2}\right\|^{1 / 2}\left\|A_{\varepsilon} u^{2}\right\|^{1 / 2}\left\|u^{3}\right\|
$$

for $u^{1} \in D\left(A_{\varepsilon}^{1 / 2}\right), u^{2} \in D\left(A_{\varepsilon}\right)$, and $u^{3} \in H_{\varepsilon}$. We now state the following results which do not use the decomposition $u=M u+(I-M) u$. We assume that $0<\varepsilon \leq 1$.

Theorem 7. Let $p$ and $r$ be two real numbers satisfying $-1<p<0$ and $r>-3$, and let $\widetilde{C}_{1}$ and $\widetilde{C}_{2}$ be two positive constants. Then there exists $\varepsilon_{0}>0$ such that, for $0<\varepsilon \leq \varepsilon_{0}$, whenever $u_{0} \in D\left(A_{\varepsilon}^{1 / 2}\right), f \in L^{\infty}\left((0, \infty), L^{2}\left(Q_{3}\right)\right)$ satisfy

$$
\left\|A_{\varepsilon}^{1 / 2} u_{0}\right\|^{2} \leq \widetilde{C}_{1} \varepsilon^{p}, \quad\left\|\mathbb{P}_{\varepsilon} f\right\|_{\infty}^{2} \leq \widetilde{C}_{2} \varepsilon^{r}
$$

then (2.5) has a solution $u$ that belongs to $C^{0}\left([0, \infty), V_{\varepsilon}^{1}\right)$ and we have

$$
\left\|A_{\varepsilon}^{1 / 2} u(t)\right\|^{2} \leq \exp \left(-\nu C_{11}^{-2} \varepsilon^{-2} t / 2\right) \widetilde{C}_{1} \varepsilon^{p}+2 C_{11}^{2} \widetilde{C}_{2} \nu^{-2} \varepsilon^{2+r}, \quad t \geq 0 .
$$

Proof. ${ }^{7}$ We set

$$
R_{0}^{2}=\widetilde{C}_{1} \varepsilon^{p}+2 C_{11}^{2} \widetilde{C}_{2} \nu^{-2} \varepsilon^{2+r}
$$

Since $R_{0}^{2} \geq\left\|A_{\varepsilon}^{1 / 2} u_{0}\right\|^{2}$, it follows from Lemma 3.0 that there is a time $T^{0}>0$ such that

$$
\left\|A_{\varepsilon}^{1 / 2} u(t)\right\|^{2} \leq 2 R_{0}^{2}, \quad 0 \leq t<T^{0} .
$$

Without loss of generality, we let $\left[0, T^{0}\right)$ denote the maximal time interval for which (7.5) is valid. If $T^{0}<\infty$, then we must have

$$
\left\|A_{\varepsilon}^{1 / 2} u\left(T^{0}\right)\right\|^{2}=2 R_{0}^{2} \text {. }
$$

By taking the scalar product of (2.5) with $A_{\varepsilon} u$ and using (7.2) and (7.4), we obtain, for $0 \leq t \leq T^{0}$,

$$
\frac{d}{d t}\left\|A_{\varepsilon}^{1 / 2} u\right\|^{2}+\nu\left\|A_{\varepsilon} u\right\|^{2} \leq \frac{1}{\nu}\left\|\mathbb{P}_{\varepsilon} f\right\|_{\infty}^{2}+2 C_{12} C_{11}^{1 / 2} \varepsilon^{1 / 2}\left\|A_{\varepsilon}^{1 / 2} u\right\|\left\|A_{\varepsilon} u\right\|^{2} .
$$

For $0 \leq t \leq T^{0}$, we have

$$
2 C_{12} C_{11}^{1 / 2} \varepsilon^{1 / 2}\left\|A_{\varepsilon}^{1 / 2} u\right\| \leq 2 \sqrt{2} C_{12} C_{11}^{1 / 2} \varepsilon^{1 / 2}\left(\widetilde{C}_{1}^{1 / 2} \varepsilon^{p / 2}+\sqrt{2} C_{11} \widetilde{C}_{2}^{1 / 2} \nu^{-1} \varepsilon^{1+r / 2}\right),
$$

which goes to 0 as $\varepsilon \rightarrow 0^{+}$. Consequently there is a positive number $\varepsilon_{0}$ such that

$$
2 \sqrt{2} C_{12} C_{11}^{1 / 2} \varepsilon^{1 / 2} R_{0} \leq \frac{\nu}{2} .
$$

For $0<\varepsilon \leq \varepsilon_{0}$, we deduce from (7.7), (7.8), and (7.2) that

$$
\frac{d}{d t}\left\|A_{\varepsilon}^{1 / 2} u\right\|^{2}+\frac{\nu \varepsilon^{-2}}{2 C_{11}^{2}}\left\|A_{\varepsilon}^{1 / 2} u\right\|^{2} \leq \frac{1}{\nu}\left\|\mathbb{P}_{\varepsilon} f\right\|_{\infty}^{2}, \quad 0 \leq t \leq T^{0}
$$

\footnotetext{
${ }^{7}$ This proof of Theorem 7 is, in fact, a small data argument.
} 
which, by the Gronwall inequality implies that

$$
\left\|A_{\varepsilon}^{1 / 2} u\right\|^{2} \leq \exp \left(-\nu C_{11}^{-2} \varepsilon^{-2} t / 2\right)\left\|A_{\varepsilon}^{1 / 2} u_{0}\right\|^{2}+\frac{2 C_{11}^{2} \varepsilon^{2}}{\nu^{2}}\left\|\mathbb{P}_{\varepsilon} f\right\|_{\infty}^{2}
$$

or

$$
\left\|A_{\varepsilon}^{1 / 2} u\right\|^{2} \leq \exp \left(-\nu C_{11}^{-2} \varepsilon^{-2} t / 2\right) \widetilde{C}_{1} \varepsilon^{p}+\frac{2 C_{11}^{2} \widetilde{C}_{2}}{\nu^{2}} \varepsilon^{2+r}
$$

for $0 \leq t \leq T^{0}$. From (7.9), it follows that

$$
\left\|A_{\varepsilon}^{1 / 2} u\left(T^{0}\right)\right\|^{2} \leq R_{0}^{2}<2 R_{0}^{2}
$$

which contradicts (7.6). Therefore $T^{0}=\infty$.

Remark. Like in $\S 4$ (see Theorem 2), one can show that, under the hypotheses of Theorem 7, if moreover $\mathbb{P}_{\varepsilon} f$ belongs to

$$
C^{0}\left([0, \infty), H_{\varepsilon}\right) \cap W^{1, \infty}\left((0, \infty), D\left(A_{\varepsilon}^{-1 / 2}\right)\right),
$$

then the solution $u(t)$ of $(2.5)$ belongs to $C^{0}\left((0, \infty), V_{\varepsilon}^{2}\right)$. Let $S_{\varepsilon}\left(\mathbb{P}_{\varepsilon} f, t\right) u_{0}$ denote the strong solution of $(2.5)$ with initial data $u_{0} \in V_{\varepsilon}^{1}$, and let $\mathscr{B}_{\varepsilon}^{1}=$ $\left\{u \in V_{\varepsilon}^{1} ;\left\|A_{\varepsilon}^{1 / 2} u\right\|^{2} \leq \widetilde{C}_{1} \varepsilon^{p}+2 C_{11}^{2} \widetilde{C}_{2} \nu^{-2} \varepsilon^{2+r}\right\}$. As in $\S 4$ (see Theorem 2) one can show that, under the assumptions of Theorem 7, if in addition, $f \in W\left(Q_{3}\right)$ is chosen so that $\mathbb{P}_{\varepsilon} f \in W^{1, \infty}\left((0, \infty) ; H_{\varepsilon}\right)$ and $H^{+}(f)$ is compact, then for any $\tau>0$ there is a compact subset $K(\tau)$ of $V_{\varepsilon}^{2}$ such that

$$
S_{\varepsilon}\left(\mathbb{P}_{\varepsilon} f, t\right) \mathscr{B}_{\varepsilon}^{1} \subset K(\tau), \quad t \geq \tau .
$$

The results below are more interesting than Theorem 7 . We recall that $\pi_{\varepsilon}\left(u_{0}, \mathbb{P}_{\varepsilon} f, \tau\right)=\left(S_{\varepsilon}\left(\mathbb{P}_{\varepsilon} f, \tau\right) u_{0},\left(\mathbb{P}_{\varepsilon} f\right)_{\tau}\right)$ denote the skew-product semiflow generated by the strong solutions of (2.5).

Corollary 7.1. Assume that the hypotheses of Theorem 7 hold and that $f \in$ $W\left(Q_{3}\right)$ is chosen so that $\mathbb{P}_{\varepsilon} f$ belongs to $W^{1, \infty}\left((0, \infty), H_{\varepsilon}\right)$ and $H^{+}(f)$ is compact. Let $\varepsilon_{0}>0$ be given by Theorem 7. Then, for $0<\varepsilon \leq \varepsilon_{0}$, the skewproduct semiflow $\pi_{\varepsilon}\left(\cdot, \mathbb{P}_{\varepsilon} f, \tau\right)$ has a unique maximal compact (local) attractor $\mathfrak{A}_{\varepsilon}$ included in $\mathscr{B}_{\varepsilon}^{1} \times \omega\left(\mathbb{P}_{\varepsilon} f\right)$ which attracts $\mathscr{B}_{\varepsilon}^{1} \times H^{+}\left(\mathbb{P}_{\varepsilon} f\right)$ in the space $V_{\varepsilon}^{1} \times$ $\mathbb{P}_{\varepsilon} W\left(Q_{3}\right)$. Futhermore,

$$
\mathfrak{A}_{\varepsilon} \subset\left\{u \in V_{\varepsilon}^{1}:\left\|A_{\varepsilon}^{1 / 2} u\right\|^{2} \leq 2 C_{11}^{2} \widetilde{C}_{2} \nu^{-2} \varepsilon^{2+r}\right\} \times \omega\left(\mathbb{P}_{\varepsilon} f\right) .
$$

Moreover, $\mathfrak{A}_{\varepsilon}$ is bounded and compact in $V_{\varepsilon}^{2} \times \omega\left(\mathbb{P}_{\varepsilon} f\right)$ and attracts the bounded set $\left(\mathscr{B}_{\varepsilon}{ }^{1} \cap V_{\varepsilon}^{2}\right) \times H^{+}\left(\mathbb{P}_{\varepsilon} f\right)$ in the space $V_{\varepsilon}^{2} \times \mathbb{P}_{\varepsilon} W\left(Q_{3}\right)$. Finally, the attractor $\mathfrak{A}_{\varepsilon}$ is the global attractor for the Leray solutions of (2.5).

Proof. The first part of this theorem is proved in the same way as Theorem 4. We will only give the argument that $\mathfrak{A}_{\varepsilon}$ is the global attractor for the Leray solutions of (2.5), i.e., the weak solutions of (2.5) that satisfy the energy estimate

$$
\|u(t)\|^{2}-\|u(0)\|^{2}+\nu \int_{0}^{t}\left\|A_{\varepsilon}^{1 / 2} u(s)\right\|^{2} d s \leq \frac{t}{\nu}\left\|A_{\varepsilon}^{-1 / 2} \mathbb{P}_{\varepsilon} f\right\|_{\infty}^{2}, \quad t>0
$$


From (7.10) and (7.2) we infer that

$$
\|u(t)\|^{2}-\|u(0)\|^{2}+\nu \int_{0}^{t}\left\|A_{\varepsilon}^{1 / 2} u(s)\right\|^{2} d s \leq \frac{C_{11}^{2} \varepsilon^{2}}{\nu} t\left\|A_{\varepsilon}^{-1 / 2} \mathbb{P}_{\varepsilon} f\right\|_{\infty}^{2},
$$

which implies that

$$
\frac{1}{t} \int_{0}^{t}\left\|A_{\varepsilon}^{1 / 2} u(s)\right\|^{2} d s \leq \frac{\nu^{-1}}{t}\left\|u_{0}\right\|^{2}+C_{11}^{2} \widetilde{C}_{2} \nu^{-2} \varepsilon^{2+r}, \quad t>0 .
$$

Therefore, for $T=C_{11}^{-2} \widetilde{C}_{2}^{-1} \nu \varepsilon^{-(2+r)}\left\|u_{0}\right\|^{2}$, there is a time $t_{0}, 0 \leq t_{0} \leq T$, such that

$$
\left\|A_{\varepsilon}^{1 / 2} u\left(t_{0}\right)\right\|^{2} \leq 2 C_{11}^{2} \widetilde{C}_{2} \nu^{-2} \varepsilon^{2+r},
$$

that is, $u\left(t_{0}\right)$ belongs to $\mathscr{B}_{\varepsilon}^{1}$ and, according to the proof of Theorem 7,u(t) is regular for all $t \geq t_{0}$. This implies that $\mathfrak{A}_{\varepsilon}$ is the global attractor for the Leray solutions of (2.5).

Corollary 7.2. Assume that the hypotheses of Corollary 7.1 hold. Then we have

$$
\sup _{(u, h) \in \mathfrak{A}_{\varepsilon}}\|u\| \rightarrow 0 \quad \text { as } \varepsilon \rightarrow 0 .
$$

If, in addition, $r>-2$, then the first components of the attractors $\mathfrak{A}_{\varepsilon}$ converge to 0 in $D\left(A_{\varepsilon}^{1 / 2}\right)$, i.e.,

$$
\sup _{(u, h) \in \mathfrak{A}_{\varepsilon}}\left\|A_{\varepsilon}^{1 / 2} u\right\| \rightarrow 0 \quad \text { as } \varepsilon \rightarrow 0
$$

Proof. Property (7.13) is an obvious consequence of Theorem 7 and Corollary 7.1, and property (7.12) is a direct consequence of Corollary 7.1 and (7.2). Indeed, we have

$$
\frac{d}{d t}\|u\|^{2}+\frac{\nu \varepsilon^{-2}}{C_{11}^{2}}\|u\|^{2} \leq \frac{C_{11}^{2} \varepsilon^{2}}{\nu^{2}}\left\|\mathbb{P}_{\varepsilon} f\right\|^{2}, \quad t>0
$$

which by Gronwall inequality implies that

$$
\|u\|^{2} \leq\left(\exp \left(-\nu C_{11}^{-2} \varepsilon^{-2} t\right)\right)\left\|u_{0}\right\|^{2}+C_{11}^{4} \widetilde{C}_{2} \nu^{-3} \varepsilon^{4+r}, \quad t \geq 0
$$

Now (7.12) follows from (7.14), the fact that $r>-3$, and the invariance of $\mathfrak{A}_{\varepsilon}$.

\section{Appendix: Proofs of Auxiliary estimates}

In this section we give the proof of the estimates (3.3) in the case of periodic boundary conditions and of the corresponding estimates in the case of other boundary conditions. We use $c_{1}, c_{2}, \ldots$ to denote constants which do not depend on $\varepsilon$ for $0<\varepsilon \leq 1$.

8.1. Periodic boundary conditions. We will keep the notation of $\S \S 2$ and 3 . Let us begin with the following lemma. 
Lemma 8.1. For any $q, 2 \leq q \leq 6$, there exist two positive constants $c_{1}$, and $c_{2}$, such that for any $w$ satisfying $M w=0$, one has

$$
\|w\|_{L^{q}\left(Q_{3}\right)} \leq c_{1} \varepsilon^{2 q^{-1}}\left(\|w\|_{H^{1}\left(Q_{3}\right)}+\varepsilon^{-1}\left\|D_{3} w\right\|_{L^{2}\left(Q_{3}\right)}\right)
$$

and

$$
\|w\|_{L^{q}\left(Q_{3}\right)} \leq c_{2} \varepsilon^{2 q^{-1}}\left\|A_{\varepsilon}^{1 / 2} w\right\|
$$

for $0<\varepsilon \leq 1$.

Proof. Inequality (8.2) is a direct consequence of (8.1) and (2.17). In order to prove (8.1) we will use two inequalities from Hale and Raugel, (1992b, Lemma 4.1 and Proposition 4.2), which can be written (in the notation of $\S 2$ ) as

$$
\|w\|_{L^{2}\left(Q_{3}\right)} \leq c_{3} \varepsilon\left(\|w\|_{H^{1}\left(Q_{3}\right)}+\varepsilon^{-1}\left\|D_{3} w\right\|_{L^{2}\left(Q_{3}\right)}\right)
$$

and

$$
\|w\|_{L^{6}\left(Q_{3}\right)} \leq c_{4} \varepsilon^{1 / 3}\left(\|w\|_{H^{1}\left(Q_{3}\right)}+\varepsilon^{-1}\left\|D_{3} w\right\|_{L^{2}\left(Q_{3}\right)}\right)
$$

whenever $M w=0$. Inequality (8.1) is then obtained by interpolation between (8.3) and (8.4). (Note that inequality (8.1) could also be derived by replacing $q=6$ in the proof of Hale and Raugel (1992b, Proposition 4.2) by any $q$, $2 \leq q \leq 6$.)

The next step is to prove the following result.

Lemma 8.2. There exist positive constants $c_{5}, c_{6}$, and $c_{7}$ such that for all $u^{1} \in$ $D\left(A_{\varepsilon}^{1 / 2}\right), u^{2} \in D\left(A_{\varepsilon}\right)$, and $u^{3} \in H_{\varepsilon}$ the following hold:

(1) If $M u^{1}=0$, then

$$
\left|b_{\varepsilon}\left(u^{1}, u^{2}, u^{3}\right)\right| \leq c_{5} \varepsilon^{1 / 3}\left\|A_{\varepsilon}^{1 / 2} u^{1}\right\|\left\|A_{\varepsilon}^{1 / 2} u^{2}\right\|^{1 / 2}\left\|A_{\varepsilon} u^{2}\right\|\left\|u^{3}\right\| .
$$

(2) If $M u^{2}=0$, then

$$
\left|b_{\varepsilon}\left(u^{1}, u^{2}, u^{3}\right)\right| \leq c_{6} \varepsilon^{1 / 6}\left\|A_{\varepsilon}^{1 / 2} u^{1}\right\|\left\|A_{\varepsilon}^{1 / 2} u^{2}\right\|^{1 / 2}\left\|A_{\varepsilon} u^{2}\right\|\left\|u^{3}\right\| .
$$

(3) If $M u^{1}=M u^{2}=0$, then

$$
\left|b_{\varepsilon}\left(u^{1}, u^{2}, u^{3}\right)\right| \leq c_{7} \varepsilon^{1 / 2}\left\|A_{\varepsilon}^{1 / 2} u^{1}\right\|\left\|A_{\varepsilon}^{1 / 2} u^{2}\right\|^{1 / 2}\left\|A_{\varepsilon} u^{2}\right\|\left\|u^{3}\right\| .
$$

Proof. Let us recall that

$$
b_{\varepsilon}\left(u^{1}, u^{2}, u^{3}\right)=\sum_{i, j=1}^{3} \int_{Q_{3}} \varepsilon^{-\{i\}} u_{i}^{1} D_{i} u_{j}^{2} u_{j}^{3} d x
$$

where $\{1\}=\{2\}=0$ and $\{3\}=1$. Using the Hölder inequality several times, we obtain

$$
\left|b_{\varepsilon}\left(u^{1}, u^{2}, u^{3}\right)\right| \leq \sum_{i, j=1}^{3}\left\|u_{i}^{1}\right\|_{L^{6}\left(Q_{3}\right)}\left\|\varepsilon^{-\{i\}} D_{i} u_{j}^{2}\right\|_{L^{2}\left(Q_{3}\right)}^{1 / 2}\left\|\varepsilon^{-\{i\}} D_{i} u_{j}^{2}\right\|_{L^{6}\left(Q_{3}\right)}^{1 / 2}\left\|u_{j}^{3}\right\|_{L^{2}\left(Q_{3}\right)}
$$



that

Assume now that $M u^{1}=0$. Then we deduce from (2.17), (8.2), and (8.8)

$$
\left|b_{\varepsilon}\left(u^{1}, u^{2}, u^{3}\right)\right| \leq c_{8} \varepsilon^{1 / 3}\left\|A_{\varepsilon}^{1 / 2} u^{1}\right\|\left\|A_{\varepsilon}^{1 / 2} u^{2}\right\|^{1 / 2}\left\|u^{3}\right\|\left(\sum_{i=1}^{3}\left\|\varepsilon^{-\{i\}} D_{i} u^{2}\right\|_{H^{1}\left(Q_{3}\right)}\right)
$$

and (8.5) is now a direct consequence of (8.9) and (2.18).

Assume next that $M u^{2}=0$. Then obviously, $M D_{i} u^{2}=0$ for $i=1,2$. Since $u^{2}$ is periodic with respect to the third variable, we also have $M D_{3} u^{2}=0$. Therefore, we can apply inequality $(8.1)$ to $w=\varepsilon^{-\{i\}} D_{i} u_{j}^{2}$ to obtain (8.10)

$$
\left\|\varepsilon^{-\{i\}} D_{i} u_{j}^{2}\right\|_{L^{6}\left(Q_{3}\right)}^{1 / 2} \leq c_{9} \varepsilon^{1 / 6}\left(\left\|\varepsilon^{-\{i\}} D_{i} u_{j}^{2}\right\|_{H^{1}\left(Q_{3}\right)}+\varepsilon^{-1}\left\|\varepsilon^{-\{i\}} D_{3} D_{i} u_{j}^{2}\right\|_{L^{2}\left(Q_{3}\right)}\right)^{1 / 2} \text {. }
$$

The estimate (8.6) is a direct consequence of (8.8), (8.10), and (2.18).

The case $M u^{1}=M u^{2}=0$ is a combination of the above, and (8.7) is a straightforward consequence of the inequalities (8.2), (8.8), (8.10), (2.17), and (2.18).

Note that (8.7) establishes the first inequality in (3.3). In order to prove the other two inequalities in (3.3), we need the following results.

Lemma 8.3. The following statements are valid:

(1) For any real numbers $r$ and $\theta$, satisfying $2 \leq r \leq 6, \frac{1}{2} \leq \theta \leq 1$, and $r \theta-6(1-\theta)>0$, there exists a positive constant $c_{10}=c_{10}(r, \theta)$ such that, for any $w \in D\left(A_{\varepsilon}\right)$ with $M w=0$, and any $u^{2} \in D\left(A_{\varepsilon}^{1 / 2}\right)$ and any $u^{3}$ in $H_{\varepsilon}$, one has

$$
\left|b_{\varepsilon}\left(w, u^{2}, u^{3}\right)\right| \leq c_{10} \varepsilon^{2(1-\theta) / r}\left\|A_{\varepsilon} w\right\|^{\theta}\left\|A_{\varepsilon}^{1 / 2} w\right\|^{1-\theta}\left\|A_{\varepsilon}^{1 / 2} u^{2}\right\|\left\|u^{3}\right\| .
$$

(2) For any real number $q, 2<q \leq 6$, there exists a positive constant $c_{11}=c_{11}(q)$ such that, for any $w \in D\left(A_{\varepsilon}\right)$ with $M w=0$, and any $v \in$ $\mathscr{R}(M) \cap D\left(A_{\varepsilon}^{1 / 2}\right)$ and any $u \in H_{\varepsilon}$, we have

$$
\left|b_{\varepsilon}(v, w, u)\right| \leq c_{11} \varepsilon^{1 / q}\left\|A_{\varepsilon}^{1 / 2} v\right\|\left\|A_{\varepsilon}^{1 / 2} w\right\|^{1 / 2}\left\|A_{\varepsilon} w\right\|^{1 / 2}\|u\| .
$$

Proof. Using the inequalities (2.17) and the Cauchy-Schwarz inequality, we obtain

$$
\left|b_{\varepsilon}\left(w, u^{2}, u^{3}\right)\right| \leq \sum_{i, j=1}^{3}\left\|w_{i}\right\|_{L^{\infty}\left(Q_{3}\right)}\left\|\varepsilon^{-\{i\}} D_{i} u_{j}^{2}\right\|_{L^{2}\left(Q_{3}\right)}\left\|u_{j}^{3}\right\|_{L^{2}\left(Q_{3}\right)},
$$

or

$$
\left|b_{\varepsilon}\left(w, u^{2}, u^{3}\right)\right| \leq c_{12}\|w\|_{L^{\infty}\left(Q_{3}\right)}\left\|A_{\varepsilon}^{1 / 2} u^{2}\right\|\left\|u^{3}\right\| .
$$

It is well known that, for any $p>3$, there exists a positive constant $c_{12}$ such that

$$
\|w\|_{L^{\infty}\left(Q_{3}\right)} \leq c_{12}\|w\|_{W^{1, p}\left(Q_{3}\right)} .
$$


Now, using a Gagliardo-Nirenberg inequality (see Friedman (1964, Theorem 10.1) for instance), we obtain, for $\frac{1}{2} \leq \theta \leq 1$ and $r\left(\theta-2+\frac{6}{p}\right)=6(1-\theta)$, that

$$
\|w\|_{W^{1, p}\left(Q_{3}\right)} \leq c_{13}\|w\|_{H^{2}\left(Q_{3}\right)}^{\theta}\|w\|_{L^{r}\left(Q_{3}\right)}^{1-\theta}
$$

where $c_{13}$ is a positive constant depending only on $r, \theta, p$. Combining the inequalities (8.14) and (8.15), we see that actually, for any real numbers $r, \theta$, satisfying $2 \leq r \leq 6, \frac{1}{2} \leq \theta \leq 1$, and $r \theta-6(1-\theta)>0$, we have

$$
\|w\|_{L^{\infty}\left(Q_{3}\right)} \leq c_{14}\|w\|_{H^{2}\left(Q_{3}\right)}^{\theta}\|w\|_{L^{r}\left(Q_{3}\right)}^{1-\theta}
$$

where $c_{14}$ is a positive constant depending only on $r, \theta$. Now the estimate (8.11) is a direct consequence of the inequalities (8.13), (8.16), (8.2), and (2.18).

Let us now prove the estimate (8.12). Using a Hölder inequality, we obtain, for any $1<\tilde{q} \leq 3$, that

$$
\left|b_{\varepsilon}(v, w, u)\right| \leq \sum_{i, j=1}^{3}\|v\|_{L^{4 p}\left(Q_{3}\right)}\left\|\varepsilon^{-\{i\}} D_{i} w\right\|_{L^{2 \dot{q}}\left(Q_{3}\right)}^{1 / 2}\left\|\varepsilon^{-\{i\}} D_{i} w\right\|_{L^{2}\left(Q_{3}\right)}^{1 / 2}\left\|u_{j}\right\|_{L^{2}\left(Q_{3}\right)},
$$

where $p=\frac{\tilde{q}}{\tilde{q}-1}$. Let us point out that the inequality (8.17) has a meaning since the vector $v$ depends only on the variables $x_{1}, x_{2}$ and therefore belongs to any space $L^{4 p}\left(Q_{3}\right), \frac{1}{4} \leq p<+\infty$, as soon as it belongs to $H^{1}\left(Q_{3}\right)$. As in the proof of Proposition 8.2, we remark that $M D_{i} w=0, i=1,2,3$; whence we may apply the inequality $(8.1)$ to $w=\varepsilon^{-\{i\}} D_{i} w$. Using the estimate $(2.18)$ in addition we obtain

$$
\left|b_{\varepsilon}(v, w, u)\right| \leq c_{15} \varepsilon^{1 / 2 \tilde{q}}\left\|A_{\varepsilon}^{1 / 2} v\right\|\left\|A_{\varepsilon} w\right\|^{1 / 2}\left\|A_{\varepsilon}^{1 / 2} w\right\|^{1 / 2}\|u\|,
$$

where $c_{15}$ is a positive constant depending only on $\tilde{q}$. By replacing $2 \tilde{q}$ with $q$ we see that (8.12) follows from (8.18).

The second estimate in (3.3) is simply the estimate (8.11) in the particular case where $r=6, \theta=\frac{17}{32}$. Likewise the third estimate (3.3) is derived from (8.12) by choosing $q=4$.

8.2. Other boundary conditions. In the proofs of $\S 8.1$, we never used the fact that the boundary conditions on $\partial Q_{2} \times(0,1)$ were periodic ones. In particular, the estimate $(8.1)$ is independent of the boundary conditions. Therefore, by using (2.17) and (2.18), one easily checks that Lemma 8.1 and Propositions 8.2 and 8.3 still hold if we replace the periodic boundary conditions on $\partial Q_{3}$ by homogeneous Dirichlet boundary conditions on $\partial Q_{2} \times(0,1)$ and periodic boundary conditions on $\left(Q_{2} \times\{0\}\right) \cup\left(Q_{2} \times\{1\}\right)$. Hence the estimates (3.3) are still true in this case.

Finally, let us consider the case where we have homogeneous Dirichlet boundary conditions on $\partial Q_{3}$. Arguing as in Hale and Raugel (1992b, Lemma 6.1) and in Lemma 8.1, one can prove the following result:

Lemma 8.4. For any $q, 2 \leq q \leq 6$, there exists a positive constant $c_{16}$ such that, for any $u \in H^{1}\left(Q_{3}\right)$ with $u=0$ on $\left(Q_{2} \times\{0\}\right) \cup\left(Q_{2} \times\{1\}\right)$, one has

$$
\|u\|_{L^{q}\left(Q_{3}\right)} \leq c_{16} \varepsilon^{2 / q}\left(\|u\|_{H^{1}\left(Q_{3}\right)}+\varepsilon^{-1}\left\|D_{3} u\right\|_{L^{2}\left(Q_{3}\right)}\right) \text {. }
$$


This lemma enables us to prove the following result.

Lemma 8.5. There exists a positive constant $c_{17}$ such that, for $u^{1} \in D\left(A_{\varepsilon}^{1 / 2}\right)$, $u^{2} \in D\left(A_{\varepsilon}\right)$ and $u^{2} \in H_{\varepsilon}$, one has

$$
\begin{aligned}
\left|b_{\varepsilon}\left(u^{1}, u^{2}, u^{3}\right)\right| \leq & c_{17} \varepsilon^{1 / 2}\left\|A_{\varepsilon}^{1 / 2} u^{1}\right\|\left\|A_{\varepsilon}^{1 / 2} u^{2}\right\|^{1 / 2}\left\|u^{3}\right\| \\
& \times\left(\sum_{i=1}^{3}\left\|\varepsilon^{-\{i\}} D_{i} u^{2}\right\|+\varepsilon^{-1}\left\|\varepsilon^{-\{i\}} D_{3} D_{i} u^{2}\right\|\right)^{1 / 2} .
\end{aligned}
$$

Proof. From (8.8), (8.19), and (2.17), we deduce that

$$
\left|b_{\varepsilon}\left(u^{1}, u^{2}, u^{3}\right)\right| \leq c_{18} \varepsilon^{1 / 3}\left\|A_{\varepsilon}^{1 / 2} u^{1}\right\|\left\|u^{3}\right\|\left\|A_{\varepsilon}^{1 / 2} u^{2}\right\|^{1 / 2}\left(\sum_{i, j=1}^{3}\left\|\varepsilon^{-\{i\}} D_{i} u_{j}^{2}\right\|_{L^{6}\left(Q_{3}\right)}^{1 / 2}\right)
$$

It remains to estimate $\left\|\varepsilon^{-\{i\}} D_{i} u_{j}^{2}\right\|_{L^{6}\left(Q_{3}\right)}^{1 / 2}$ for $1 \leq i, j \leq 3$. Since $D_{i} u_{j}$ is equal to zero on $\left(Q_{2} \times\{0\}\right) \cup\left(Q_{2} \times\{1\}\right)$ if $i=1,2$ and since $M D_{3} u_{j}=0$, for $1 \leq j \leq 3$, one can apply Lemmas 8.4 and 8.1 to $D_{i} u_{j}, i=1,2$, and $D_{3} u_{j}$, respectively, for $1 \leq j \leq 3$. From (8.1), (8.18), and (8.21), we at once infer the estimate (8.20).

\section{ACKNOWLEDGMENT}

We express our sincere appreciation to Ciprian Foiaş, Jack Hale, and Roger Temam for their helpful suggestions on this paper. We are especially grateful to Ciprian Foias for observing that our arguments show that $\mathfrak{A}_{\varepsilon}$ is the global attractor for the Leray solutions of the 3DNS.

\section{REFERENCES}

A. V. Babin and M. I. Vishik (1989), Attractors of evolutionary equations, Nauka. (Russian)

L. Caffarelli, R. Kohn, and L. Nirenberg (1982), Partial regularity of suitable weak solutions of the Navier-Stokes equations, Comm. Pure Appl. Math. 35, 771-831.

P. Constantin and C. Foiaş (1988), Navier-Stokes equations, Univ. of Chicago Press, Chicago.

M. Dauge (1984), Réguliarité et singularités des systèmes de Stokes et Navier-Stokes dans des domaines non réguliers de $\mathbb{R}^{2}$ ou $\mathbb{R}^{3}$, Séminaire d'Equations aux Dérivées Partielles de Nantes-1984.

(1989), Stationary Stokes and Navier-Stokes systems on two-or three-dimensional domains with corners, Part I: Linearized equations, SIAM J. Math Anal. 20, 74-97

C. Foiaş, C. Guillopé, and R. Temam (1981), New and a priori estimates for Navier-Stokes equations in dimension 3, Comm. Partial Differential Equations 6, 329-359.

C. Foiaş, O. Manley, and R. Temam (1987), Attractors for the Benard problem: Existence and physical bounds of their fractal dimension, Nonlinear Anal. 11, 939-967.

C. Foiaş, G. R. Sell, and R. Temam (1988), Inertial manifolds for nonlinear evolutionary equations, J. Differential Equations 73, 309-353.

C. Foias and R. Temam (1979), Some analytic and geometric properties of the solutions of the Navier-Stokes equations, J. Math. Pures Appl. 58, 339-368.

(1987), The connection between the Navier-Stokes equations, dynamical systems, and turbulence theory, Directions in Partial Differential Equations, Academic Press, New York, pp. 55-73. 
H. Fujita and T. Kato (1964), On the Navier-Stokes initial value problem. I, Arch. Rational Mech. Anal. 16, 269-315.

A. Friedman (1964), Partial differential equations of parabolic type, Prentice-Hall, Englewood Cliffs, NJ.

Y. Giga (1988), Book review, Bull. Amer. Math. Soc. 19, 337-340.

J. K. Hale (1988), Asymptotic behavior of dissipative systems, Math. Surveys Monographs, vol. 25 Amer. Math. Soc., Providence, RI.

J. K. Hale and G. Raugel (1990), Partial differential equations on thin domains, Differential Equations and Mathematical Physics, Proceedings of International Conference in Alabama, March 1990 (C. Bennewitz, Ed.), Academic Press, New York, pp. 63-98.

(1992a), Reaction diffusion equation on thin domains, J. Math. Pures Appl. 71, 33-95.

(1992b), A damped hyperbolic equation on thin domains, Trans. Amer. Math. Soc. 329, 185219.

D. Henry (1981), Geometric theory of semilinear parabolic equations, Lecture Notes in Math., vol. 840 , Springer-Verlag, New York.

E. Hopf (1951), Über die Anfangswertaufgabe für die hydrodynamischen Grundgleichungen, Math. Nachr. 4, 213-231.

A. Kiselev and O. A. Ladyzhenskaya (1957), On the existence and uniqueness of the solution of the non-stationary problem for a viscous incompressible fluid, Izv. Akad. Nauk SSSR Ser. Mat. 21, 655-680.

G. Komatsu (1980), Global analyticity up to the boundary of solutions of the Navier-Stokes equation, Comm. Pure Appl. Math. 33, 545-566.

O. A. Ladyzhenskaya (1969), The mathematical theory of viscous incompressible flow, 2nd ed., Gordon and Breach, New York.

(1970), Unique solvability in the large of three dimensional Cauchy problem for the NavierStokes equations in the presence of axial symmetry, Boundary Value Problem of Mathematical Physics and Related Aspects of Function Theory, Steklov Math. Inst., Leningrad, pp. 70-79.

(1972), On the dynamical system generated by the Navier-Stokes equations, J. Soviet Math. 3 , 458-479.

J. Leray (1933), Etude de diverses équations intégrales nonlinéaires et de quelques problèmes que pose Thydrodynamique, J. Math. Pures Appl. 12, 1-82.

(1934a), Essai sur les mouvements plans d'un liquide visqueux que limitent des parois, J. Math. Pures Appl. 13, 331-418.

(1934b), Sur le mouvement d'un liquide visqueux emplissant l'espace, Acta Math. 63, 193-248.

J. L. Lions (1969), Quelques méthodes de résolution des problèmes aux limites non linéaires, Gauthier Villars, Paris.

J. L. Lions and G. Prodi (1959), Un théorème d'existence et d'unicité dans les équations de NavierStokes en dimensions 2, C. R. Acad. Sci. Paris Sér. I Math. 248, 3519-3521.

A. Mahalov, E. S. Titi, and S. Leibovich (1990), Invariant helical subspaces to the solution of the Navier-Stokes equations, Arch. Rational Mech. Anal. 112, 193-222.

J. Mallet-Paret (1976), Negatively invariant sets of compact maps and an extension of a theorem of Cartwright, J. Differential Equations 22, 331-348.

K. Masuda (1967), On the analyticity and unique continuation theorem for solutions of the Navier Stokes equations, Proc. Japan Acad. 43, 827-832.

R. K. Miller and G. R. Sell (1970), Volterra integral equations and topological dynamics, Mem. Amer. Math. Soc., vol. 102, Amer. Math. Soc., Providence, RI.

G. Raugel (1989), Continuity of attractors, RAIRO Modél. Math. Anal. Numér. 23, 519-533.

G. Raugel and G. R. Sell (1989), Equations de Navier-Stokes dans des domaines minces en dimension trois: régularité globale, C. R. Acad. Sci. Paris Sér. I Math. 309, 299-303. 
(1992a), Navier-Stokes equations on thin 3D domains. II: Global regularity of spatially periodic solutions, College de France Proceedings, Pitman Res. Notes Math. Ser., Pitman, New York and London.

(1992b), Navier-Stokes equations on thin 3D domains. III: Global attractors, IMA Proceedings on Dynamical Systems Approaches to Turbulence.

R. J. Sacker and G. R. Sell (1977), Lifting properties in skew-product flows with applications to differential equations, Mem. Amer. Math. Soc., vol. 190, Amer. Math. Soc., Providence, RI.

(1992), Dichotomies for linear evolutionary equations in Banach spaces, J. Differential Equations (to appear).

G. R. Sell (1967a), Nonautonomous differential equations and topological dynamics. I: The basic theory, Trans. Amer. Math. Soc. 127, 241-262.

(1967b), Nonautonomous differential equations and topological dynamics. II: Limiting equations, Trans. Amer. Math. Soc. 127, 263-283.

(1973), Differential equations without uniqueness and classical topological dynamics, J. Differential Equations 14, 42-56.

G. R. Sell and Y. You (1993), Dynamical systems and global attractors, preprint.

J. Serrin (1962), On the interior regularity of weak solutions of the Navier-Stokes equations, Arch. Rational Mech. Anal 9, 187-195.

R. Temam (1977), Navier-Stokes equations, North-Holland, Amsterdam.

(1982), Behavior at time $t=0$ of the solutions of semilinear evolution equations, J. Differential Equations 43, 73-92.

(1983), Navier-Stokes equations and nonlinear functional analysis, CBMS Regional Conference Series, No. 41, SIAM, Philadelphia.

(1988), Infinite dimensional dynamical systems in mechanics and physics, Springer-Verlag, New York.

W. von Wahl (1985), The equations of Navier-Stokes and abstract parabolic problems, Vieweg and Sohn, Braunschweig.

ABstract. We examine the Navier-Stokes equations (NS) on a thin 3-dimensional domain $\Omega_{\varepsilon}=Q_{2} \times(0, \varepsilon)$, where $Q_{2}$ is a suitable bounded domain in $\mathbb{R}^{2}$ and $\varepsilon$ is a small, positive, real parameter. We consider these equations with various homogeneous boundary conditions, especially spatially periodic boundary conditions. We show that there are large sets $\mathscr{R}(\varepsilon)$ in $H^{1}\left(\Omega_{\varepsilon}\right)$ and $\mathscr{S}(\varepsilon)$ in $W^{1, \infty}\left((0, \infty), L^{2}\left(\Omega_{\varepsilon}\right)\right)$ such that if $U_{0} \in \mathscr{R}(\varepsilon)$ and $F \in \mathscr{S}(\varepsilon)$, then (NS) has a strong solution $U(t)$ that remains in $H^{1}\left(\Omega_{\varepsilon}\right)$ for all $t \geq 0$ and in $H^{2}\left(\Omega_{\varepsilon}\right)$ for all $t>0$. We show that the set of strong solutions of (NS) has a local attractor $\mathfrak{A}_{\varepsilon}$ in $H^{1}\left(\Omega_{\varepsilon}\right)$, which is compact in $H^{2}\left(\Omega_{\varepsilon}\right)$. Furthermore, this local attractor $\mathfrak{A}_{\varepsilon}$ turns out to be the global attractor for all the weak solutions (in the sense of Leray) of (NS). We also show that, under reasonable assumptions, $\mathfrak{A}_{\varepsilon}$ is upper semicontinuous at $\varepsilon=0$.

Laboratoire d'Analyse Numérique, Bâtiment 425, Université de Paris Sud (et CNRS), 91405 Orsay Cedex, France

E-mail address: raugel@matups.matups.fr

School of Mathematics and AHPCRC, University of Minnesota, Minneapolis, Minnesota 55455

E-mail address: sell@ahpcrc.umn.edu 